\title{
Cooperative and Cognitive Communication Techniques for Wireless Networks
}

\author{
by \\ Christos Tsinos \\ Submitted to the Department of Computer Engineering and \\ Informatics \\ in fulfillment of the requirements for the degree of \\ Doctor of Philosophy \\ at the \\ University of Patras, Patras Greece
}

October 2013

(c) Christos Tsinos 2013. All rights reserved.

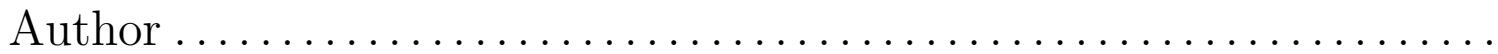

Department of Computer Engineering and Informatics

October 17, 2013

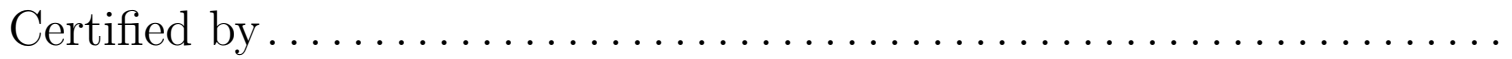

Kostas Berberidis

Professor

Thesis Supervisor 


\section{Dissertation Title:}

Cooperative and Cognitive Communication Techniques for Wireless Networks

Christos Tsinos

\section{Supervisor:}

Prof. Kostas Berberidis ${ }^{1}$

\section{Thesis Committee:}

Prof. Kostas Berberidis ${ }^{1}$

Prof. Emmanuel Varvarigos ${ }^{1}$

Dr. Athanasios Rontogiannis ${ }^{2}$

\section{Thesis Examination Committee:}

Prof. Kostas Berberidis ${ }^{1}$

Prof. Sergios Theodoridis ${ }^{3}$

Prof. Stavros Kotsopoulos ${ }^{4}$

Assistant Prof. Ioannis Krikidis ${ }^{5}$

Prof. Emmanuel Varvarigos ${ }^{1}$

Dr. Athanasios Rontogiannis ${ }^{2}$

Assistant Prof. Dimitrios-Alexandros Toumpakaris ${ }^{4}$

\footnotetext{
${ }^{1}$ Department of Computer Engineering and Informatics, University of Patras

${ }^{2}$ National Observatory of Athens

${ }^{3}$ Department of Informatics and Telecommunications, University of Athens

${ }^{4}$ Department of Electrical and Computer Engineering, University of Patras

${ }^{5}$ Department of Computer and Electrical Engineering, University of Cyprus
} 
This research has been co-financed by the European Union (European Social Fund ESF) and Greek national funds through the Operational Program "Education and Lifelong Learning" of the National Strategic Reference Framework (NSRF) - Research Funding Program: Heracleitus II. Investing in knowledge society through the European Social Fund.
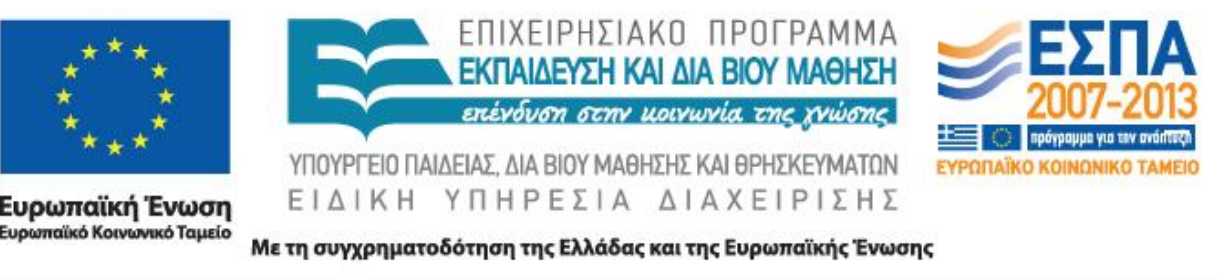


\title{
Cooperative and Cognitive Communication Techniques for Wireless Networks
}

\author{
by \\ Christos Tsinos

\begin{abstract}
Submitted to the Department of Computer Engineering and Informatics on October 17, 2013, in fulfillment of the requirements for the degree of

Doctor of Philosophy
\end{abstract}

\begin{abstract}
During the past years wireless communications have been exhibiting an increased growth rendering them the most common way for communication. The continuously increasing demand for wireless services resulted in limited availability of the wireless spectrum. To this end, Cognitive Radio (CR) techniques have been proposed in literature during the past years. The concept of CR approach is to utilize advanced radio and signal-processing technology along with novel spectrum allocation policies to enable new unlicensed wireless users to operate in the existing occupied spectrum areas without degrading the performance of the existing licensed ones. Moreover, the broadcast and fading nature of the wireless channel results in severe degradation on the performance of wireless transmissions. A solution to the problem is the use of multiple-antenna systems so as to achieve spatial diversity. However, in many cases, the communication devices' nature permit the support of multiple antennas due to size, power consumption, and hardware limitations. To this end, cooperative communications provide an alternative way to achieve spatial diversity via virtual antenna arrays formed by single antenna nodes. It is noteworthy that cooperation has an important role within the CR literature as many techniques developed within its context exploiting the benefits of cooperation in order to achieve improved performance. Therefore, the aim of the present dissertation is to develop efficient and practical cognitive, cooperative and cognitive cooperative schemes. More specifically the contributions are the following ones.

The first contribution is a novel CR communication scheme. During the past years numerous $\mathrm{CR}$ communication schemes have been presented in literature. To the best of our knowledge, the majority of them were developed assuming perfect Channel State Information (CSI) at the unlicensed user's side. There are several cases where the licensed users do not desire any interaction with the unlicensed ones. In such cases, the assumption that the unlicensed user can obtain CSI that concerns the licensed user channels is not valid and as a result the corresponding communication technique cannot be applied. Therefore, at first we propose an novel CR communication scheme that requires CSI that can be estimated in a completely blind manner. Then, the
\end{abstract}


corresponding blind estimation scheme is developed. Another significant contribution is the theoretical results that have been derived for both the perfect CSI case and the imperfect CSI case (when the blind estimation scheme is employed for obtaining the corresponding CSI). Especially, the theoretical results that concern the imperfect CSI case are some of the first ones that appear in the relevant literature, to the best of our knowledge.

The second contribution is a decentralized adaptive Eigenvalue-Based spectrum Sensing (EBSS) technique for multi-antenna CR Systems. Spectrum Sensing is a fundamental functionality in CR systems. In general, the unlicensed user employs a spectrum sensing technique in order to detect licensed user(s) activity in scenarios where the former user is permitted to establish a communication link only via spectrum areas that are temporarily free of the latter one's transmissions. EBSS techniques are known to achieve good performance and also to be applicable in a completely blind manner. In the literature so far, only batch and centralized cooperative EBSS techniques have been considered which, however, suffer from limitations that render them impractical in several cases such as, when time-varying channels are involved or continuous spectrum monitoring is required. Thus, the aim here is to develop practical cooperative adaptive versions of typical Eigenvalue-Based Spectrum Sensing (EBSS) techniques which could be applied in a completely decentralized manner and cope well in time-varying scenarios. To this end, at first, novel adaptive EBSS techniques are developed for the Maximum Eigenvalue Detector (MED), the Maximum-Minimum Eigenvalue Detector (MMED), and the Generalized Likelihood Ratio Test (GLRT) schemes, respectively, for a single-user (no cooperation) case. Then, a novel distributed subspace tracking method is proposed which enables the cooperating nodes to track the joint subspace of their received signals. Based on this method, cooperative decentralized versions of the adaptive EBSS techniques are subsequently developed that overcome the limitations of the existing batch centralized approaches.

The third contribution is a new cooperative scheme for half-duplex uplink transmission. The technique is based on a virtual MIMO structure formed by the single antenna source and relays nodes along with the multi-antenna base station which is the destination node. The new technique aims at providing increased diversity and multiplexing gains, contrariwise to existing approaches where the proposed techniques achieve increased diversity gain at the cost of severe multiplexing gain loss. The theoretical outage probability and the corresponding Diversity Multiplexing Trade-off (DMT) curve of the proposed technique are also derived.

The final contribution is two novel algorithms which enable the relay cooperation for the distributed computation of the beamforming weights in a blind and adaptive manner, without the need to forward the data to a fusion center. The proposed algorithms are constituent parts of two corresponding distributed beamforming schemes for relay networks that distribute the computational overhead equally among the relay nodes. In the first scheme, the beamforming vector is computed through minimization of the total transmit power subject to a receiver quality-of-service constraint (QoS). In the second scheme, the beamforming weights are obtained through maximization of the receiver signal-to-noise-ration (SNR) subject to a total transmit power constraint. 
The proposed approaches achieve close performance to the one of the optimal beamforming solutions derived assuming perfect channel state information at the relays' side. 


\section{Acknowledgments}

First and foremost, I would like to express my sincerest gratitude to my supervisor Prof. Kostas Berberidis for his invaluable and insightful guidance throughout the course of my diploma, master diploma and doctoral thesis. I would also like to thank, Prof. Emmanuel Varvarigos, Dr. Athanasios Rontogiannis, Prof. Sergios Theodoridis, Prof. Stavros Kotsopoulos, Assistant Prof. Ioannis Krikidis and Assistant Prof. Dimitrios-Alexandros Toumpakaris for honouring me by accepting to be my thesis committee members. During the past years I met many great people at the lab. I would like to express my gratitude to all of them for making a pleasant environment for working. I would not have been here today if it were not for the love and support of my family. I want to express my deepest thanks to them for their unconditional love, devotion and support. Finally, I would like to thank myself for being able to pull through it. As anyone that was in the same place could tell you, the road is not easy, it demands high concentration, courage and patience. Nevertheless, at the end of the day if I was to decide again if I would embark on a Phd or not, the decision would have been the same, without a second thought. After all, it was a hell of a ride...

Christos Tsinos

Patras, 17/10/2013 


\section{List of Symbols}

\begin{tabular}{|c|c|}
\hline A & Matrix \\
\hline $\mathbf{a}$ & Vector \\
\hline$|\mathbf{a}|$ & Abscissa of each entry of $\mathbf{a}$ \\
\hline $\operatorname{diag}(\mathbf{a})$ & Vector to Diagonal Matrix Operator \\
\hline$\odot$ & Element-Wise Schur-Hadamard Product \\
\hline$a$ & Scalar \\
\hline $\mathbf{A}^{H}$ & Hermitian of $\mathbf{A}$ \\
\hline $\mathbf{A}^{*}$ & Conjugate of $\mathbf{A}$ \\
\hline $\mathbf{A}^{T}$ & Reverse of $\mathbf{A}$ \\
\hline $\mathcal{P}_{1}(\mathrm{~A})$ & Principal EigenVector of $\mathbf{A}$ \\
\hline $\operatorname{tr}(\mathbf{A})$ & Trace of $\mathbf{A}$ \\
\hline$\|\mathbf{A}\|$ & $l_{2}$ Norm of $\mathbf{A}$ \\
\hline$|\mathbf{A}|$ & Determinant of $\mathbf{A}$ \\
\hline $\mathcal{N} \mathcal{U} \mathcal{L} \mathcal{L}(\mathrm{A})$ & Null Space of $\mathbf{A}$ \\
\hline$\delta \mathbf{A}$ & Perturbation Term \\
\hline $\operatorname{orth}\{\mathbf{A}\}$ & Orthonormalization of the columns of $\mathbf{A}$ \\
\hline $\operatorname{norm}\{\mathbf{A}\}$ & Normalization of the columns of $\mathbf{A}$ \\
\hline $\mathbf{I}_{M}$ & $M \times M$ Identity Matrix \\
\hline$J(\cdot, \cdot)$ & Jacobian Matrix of a Transformation \\
\hline $\mathbb{E}\{\cdot\}$ & Expected Value Operator \\
\hline$(\cdot)^{+}$ & $\max \{(\cdot), 0\}$ \\
\hline
\end{tabular}




$\begin{array}{ll}(\cdot) ! & \text { Factorial } \\ \Gamma(\cdot, \cdot) & \text { Upper Incomplete Gamma Function } \\ \gamma(\cdot, \cdot) & \text { Lower Incomplete Gamma Function } \\ \Gamma(\cdot) & \text { Ordinary Gamma Function } \\ \mathcal{I}_{(\cdot)}(\cdot) & \text { Incomplete Beta Function } \\ B(\cdot, \cdot) & \text { Beta Function } \\ G_{p, q}^{m, n} & \text { MeijerG Function } \\ \boldsymbol{\Phi}_{2}^{(2)}(\cdot ; \cdot ; \cdot, \cdot) & \text { Confluent Lauricella Hypergeometric Function } \\ (a)_{k} & \text { Pochammer symbol } \\ \mathbb{1}(\cdot) & \text { Index Function } \\ (\cdot) & \text { Binomial Term } \\ \mathcal{P}(\cdot) & \text { Probability of an Event } \\ \mathcal{P}_{f} & \text { Probability of False Alarm } \\ \mathcal{P}_{d} & \text { Probability of Detection } \\ \sim & \text { Follows the Distribution } \\ \approx & \text { Approximately Equally } \\ & \end{array}$




\section{Contents}

1 Distributed and Cooperative Techniques in Cognitive Radio Net$\begin{array}{ll}\text { works } & 17\end{array}$

1.1 Introduction . . . . . . . . . . . . . . . . . . . . . . 17

1.2 Cognitive Radio Networks . . . . . . . . . . . . . . . . . 17

1.3 Cognitive Radio Networks Schemes . . . . . . . . . . . . . . . 20

1.3.1 The Underlay Approach . . . . . . . . . . . . . . . . . . . 21

1.3.2 The Overlay Approach . . . . . . . . . . . . . . . . . . . . . 21

1.3.3 The Interweave Approach . . . . . . . . . . . . . . . . 22

1.3.4 Comparison of the Approaches . . . . . . . . . . . . . . . 23

1.4 Spectrum Sensing Techniques . . . . . . . . . . . . . . . . . . 24

1.5 Cooperative Communication Techniques . . . . . . . . . 25

1.6 Distributed Estimation . . . . . . . . . . . . . . . . . . . . . . . . . . . . . 29

1.7 Contributions and Outline $\ldots \ldots \ldots \ldots$

1.8 Conclusion . . . . . . . . . . . . . . . . . . . . . . . . . . . . . . . . . . 32

2 Blind Opportunistic Interference Alignment in MIMO Cognitive Radio Systems

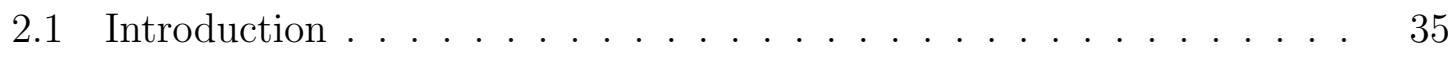

2.2 System Description . . . . . . . . . . . . . . . . . . . . 38

2.3 Opportunistic Interference Alignment _ . . . . . . . . . . . . . 39

2.3.1 The Opportunistic Scheme of the Original Approach _. . . . 39

2.3.2 The Proposed Approach . . . . . . . . . . . . . . . . 42

2.4 Blind Opportunistic Interference Alignment _ . . . . . . . . . 44 
2.4.1 Estimation of Matrices $\mathbf{Q}_{r}$ and $\mathbf{Q}_{t} \ldots \ldots \ldots \ldots$

2.4.2 Detection of the PU's Signal Subspace Rank . . . . . . . . 46

2.4.3 Estimation of Matrices $\mathbf{V}_{z}$ and $\mathbf{U}_{z} \ldots \ldots \ldots$

2.4.4 The Complete Estimation Scheme . . . . . . . . . . . . . 52

2.5 Performance Analysis . . . . . . . . . . . . . . . . . . . . . . 54

2.5 .1 Perfect CSI Case . . . . . . . . . . . . . . . . 55

2.5.2 The Imperfect CSI Case with Perfect PU Rank Knowledge . . 57

2.5.3 The Imperfect CSI Case with Imperfect Knowledge of PU's Signal Subspace Rank . . . . . . . . . . . . . . . . . 59

2.6 Simulation Results . . . . . . . . . . . . . . . . . . . . . . . . . . 62

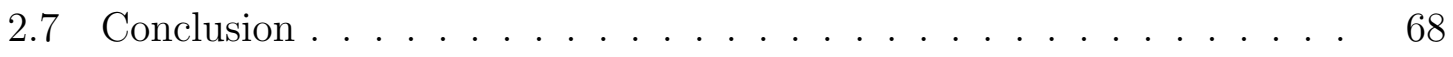

2.8 Appendices . . . . . . . . . . . . . . . . . . . . . . . . . 69

2.A Proof of Theorem $2.1 \ldots \ldots \ldots \ldots \ldots$

2.B Proof of Lemma 2.1 . . . . . . . . . . . . . . . . . 70

2.C Proof of Theorem $2.2 \ldots \ldots \ldots \ldots \ldots \ldots$

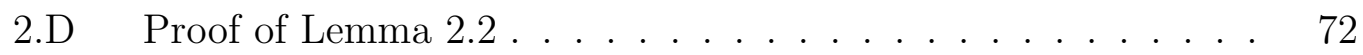

\section{Decentralized Adaptive Eigenvalue-Based Spectrum Sensing for Multi-} $\begin{array}{ll}\text { antenna Cognitive Radio Systems } & 75\end{array}$

3.1 Introduction . . . . . . . . . . . . . . . . . 75

3.2 System Description . . . . . . . . . . . . . . . . . 77

3.3 Centralized Batch Cooperative Eigenvalue-Based Spectrum Sensing • 78

3.4 Adaptive Eigenvalue-Based Spectrum Sensing for the Single SU Case 80

3.5 Test Statistics Distributions and Decision Thresholds . . . . . . . 83

3.6 Cooperative Decentralized Adaptive Eigenvalue-Based Spectrum Sensing 86 3.6.1 Related Work . . . . . . . . . . . . . . . . . . 86

3.6.2 Distributed Data Projection Method (DDPM) . . . . . . . 87

3.6.3 Cooperative EBSS techniques _ . . . . . . . . . . 90

3.7 Simulation Results . . . . . . . . . . . . . . . . . . . . . . . . . . 91

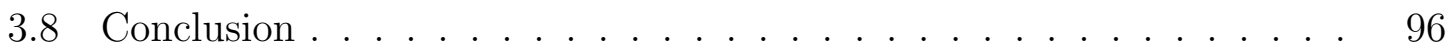


3.9 Appendix . . . . . . . . . . . . . . . . . . . . 97

3.A Derivation of the Distribution of the MMED Test Statistic under the $\mathcal{H}_{0}$ Hypothesis . . . . . . . . . . . . . . . 97

4 A Cooperative Uplink Transmission Technique with Improved DiversityMultiplexing Tradeoff $\quad 99$

4.1 Introduction . . . . . . . . . . . . . . . . . . . . . 99

4.2 System's Description . . . . . . . . . . . . . . . . . . . 101

4.3 Performance Analysis . . . . . . . . . . . . . . . . . . 105

4.3.1 Outage Probability Analysis . . . . . . . . . . . . 105

4.3.2 Diversity-Multiplexing Tradeoff Analysis . . . . . . . . . . 109

4.4 Simulations . . . . . . . . . . . . . . . . . . . . 113

4.5 Conclusion . . . . . . . . . . . . . . . . . . 116

5 Distributed Blind Adaptive Computation of Beamforming Weights for Relay Networks $\quad 119$

5.1 Introduction . . . . . . . . . . . . . . . . . . . . . . . . 119

5.2 Problem Formulation . . . . . . . . . . . . . . . . . . . . . 121

5.2 .1 System Model . . . . . . . . . . . . . . . . . . . . . . . . 121

5.2.2 Distributed Beamforming Schemes _ . . . . . . . . . . 122

5.3 Distributed Blind Estimation Scheme of the Beamforming Vector . . 124

5.3.1 The Power Minimization Problem . . . . . . . . . . . . 126

5.3.2 SNR Maximization Problem . . . . . . . . . . . . . . 127

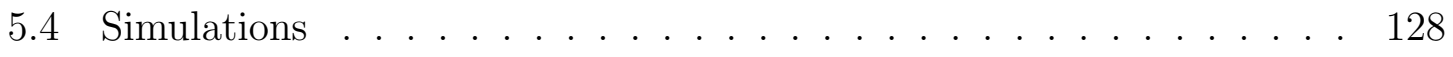

5.5 Conclusion . . . . . . . . . . . . . . . . . . . . . 130

6 Conclusions and Open Issues 133 


\section{Chapter 1}

\section{Distributed and Cooperative Techniques in Cognitive Radio Networks}

\section{$1.1 \quad$ Introduction}

In this chapter a brief overview of the relevant literature, the motivations, the contributions and the outline of the dissertation are given. In Section 1.2 general information regarding the Cognitive Radio $(\mathrm{CR})$ are presented. In Section 1.3 the approaches followed in designing the CR communication techniques are discussed. Section 1.4 presents the Spectrum Sensing techniques that constitute a core functionality of the majority of the CR systems. Section 1.5 discusses the concept of cooperative communication techniques. In Section 1.6 the distributed estimation concept is presented. In Section 1.7 the contributions of the present dissertation are summarized and an outline of the rest of the text is given. Finally, Section 1.8 concludes the work.

\subsection{Cognitive Radio Networks}

The wireless transmissions are established via a broadcast medium and therefore all the users coexisting in the same frequency band interfere with each other. During the 
last two decades, the number of wireless systems and services has grown exponentially resulting in limited availability of the the wireless spectrum. The aforementioned statement can be verified by the US National Telecommunications and Information Administration's frequency allocation chart ${ }^{1}$, which reveals that almost all frequency bands have been assigned, and there is little new bandwidth available for emerging wireless products and technologies. This limited availability of wireless spectrum led to the concept of the Cognitive Radio (CR). The devices that are built using the CR approach, utilize advanced radio and signal-processing technology along with novel spectrum allocation policies to enable new wireless users to operate in the existing occupied spectrum areas without degrading the performance of the entrenched users. A wide adoption of the CR technology could provide the required bandwidth to support the demand for higher quality and data rate products and services into the future.

A CR device should be able to collect and process information regarding the coexisting users within its spectrum. Hence, advanced sensing and signal processing capabilities are required. The latter capabilities are extensively studied in literature over the past years, so their necessity do not form a major barrier to the implementation of a CR device. The actual barrier is the requirement for significant changes in wireless spectrum allocation policy so as to enable the application of CR techniques. Unfortunately, the corresponding regulatory bodies that have to do with spectrum allocation have not shown much yearn for a change since their inception in the early 1900s. In fact, until recently, spectrum regulatory bodies such as the Federal Communications Commission (FCC) in the United States or the European Telecommunications Standards Institute (ETSI) in Europe always allocated spectral frequency blocks for specific uses and assigned licenses for these blocks to specific groups or companies. The latter approach involves a) the spectrum division to distinct bands, each one of them including a range of frequencies, b) allocating specific bands to specific communication uses, and c) determining a license for each band. Some examples of licensed frequency bands today are the radio and the television

\footnotetext{
${ }^{1}$ www.ntia.doc.gov/osmhome/allochrt.pdf
} 
bands, air traffic and control bands, and cellular and satellite bands. The obvious advantage of the licensing policy is that the licensee completely controls its assigned spectrum and as a consequence it manages unilaterally the interference among its users and therefore their Quality of Service (QoS). Up to the mid 1990s, the licenses were granted free of charge in general, based on an application process. Today, the majority of the licenses that are not for public safety or military use are granted via spectral auctions.

Apart from the licensed spectrum areas, in recent years, spectrum has been set aside in specific frequency bands that can be used without a requirement for a license by radios following a specific set of etiquette rules, such as maximum power per $\mathrm{Hz}$ or a shared channel access mechanism. These unlicensed bands exist so as to encourage innovation without the high cost associated with purchasing licensed spectrum bands via auctions. The unlicensed bands have been used for many innovating applications and the $2.4 \mathrm{GHz}$ unlicensed band currently hosts systems such as Bluetooth, $802.11 \mathrm{~b} / \mathrm{g} / \mathrm{n}$ Wifi and cordless phones. Unfortunately the excessive number of wireless devices operating in the unlicensed bands can lead to severe degradation on their performance due to the interference they cause to each other.

The spectrum allocation policy is not just limited to licensed and unlicensed bands categorization. Within both kinds of the bands, there may exist many additional wireless devices provided that these devices can exploit the CR technology so as to establish their communication links with no or minimal disruption of the communications of the coexisting non-cognitive users. The concept of CR originated as a number of various solutions to the aforementioned problem that allow cognitive communication with no or little impact on non-cognitive users. Before proceeding any further, it should be mentioned that in the CR literature it is common to reference a non-cognitive user as Primary User (PU) and a cognitive one as a Secondary User (SU). From now and on, both of the terms may be used in the text. Since its introduction in the pioneering work of J. Mitola [1], the idea of CR has evoked much enthusiasm, including within the FCC, which formed a spectrum policy task force to provide new policy recommendations that support the CR approach [2]. During the 
years, the initial ideas, evolved in various directions, leading to a variety of different approaches. However, behind these diverse CR interpretations lies a common defining feature: awareness of its environment [3]. In the information theory terminology, a $\mathrm{CR}$ is defined by the capability of acquiring and utilizing network side information that formalize as follows

A CR is a wireless communication system that intelligently utilizes any available information about a) activity, b) channel conditions, c) codebooks, or d) messages of other nodes with which it shares the spectrum.

According to the type of the available network side information along with the regulatory constraints, CR systems can be mainly divided in three categories the underlay, the overlay and the interweave one. In the following section those three categories are described in more detail.

\subsection{Cognitive Radio Networks Schemes}

As it was just mentioned, there are three major categories of CR techniques: underlay, overlay and interweave. The underlay approach, allows the SUs to operate in a way such that the interference generated to the PUs' transmissions is below a given threshold. In the overlay approach, the SUs use sophisticated signal processing and coding to maintain or improve the communication link of the PUs while also obtaining some additional bandwidth for their own communication. Finally, in the interweave systems the SUs opportunistically exploit temporary unused frequency bands by the PUs, the so-called "spectrum holes", so as to communicate without disrupting the corresponding PU transmissions. In the following subsections each one of the aforementioned approaches is described in detail, including the associated regulatory policy as well as the underlying assumptions about what network side information is available. 


\subsubsection{The Underlay Approach}

The underlay approach includes techniques that allow communication by the $\mathrm{SU}$, assuming it has knowledge of the interference caused by its transmitter to the PUs' receivers. In this setting, usually the $\mathrm{SU}(\mathrm{s})$ cannot significantly interfere with the communication of the PU(s). In more detail, in the underlay approach the SUs are allowed to transmit simultaneously to the PUs' transmissions only if the interference generated by the SUs is below some predefined threshold [4]-[7]. The interference constraint may be posed to the SUs by using multiple antennas to guide the cognitive signals away from the PUs' transmissions, or by the use of a wide bandwidth over which the cognitive signal can be spread below the noise floor and then despread at the SU receiver. The latter techniques is known from both the spread spectrum and Ultra-Wide-Band (UWB) communications. The interference generated by a SU transmitter to a PU receiver can be approximated if the SU transmitter listens to a $\mathrm{PU}$ receiver transmission, assuming that the reciprocity assumption holds. An alternative approach is the $\mathrm{SU}$ transmitter to be somewhat conservative in its output power so that the SU signal to be transmitted satisfies always the interference constraint. In the previous case, the interference constraints are quite restrictive and therefore the SUs are limited to short range communications. While the underlay approach is most common in licensed spectrum bands (e.g. UWB underlays many licensed spectral bands), it can be also employed in unlicensed bands so as to provide different types of services to different users.

\subsubsection{The Overlay Approach}

The main characteristic of the overlay approach is that the SU transmitter has knowledge of the PUs' codebooks and messages [8]-[11]. The codebook information can be obtained if, for example, the PUs employ a uniform standard for communication that is based on a publicized codebook. An alternative policy can be the periodical transmission of the PU codebooks. The knowledge concerning the PU message can be obtained by decoding the message at the SU receiver. Nevertheless, in the overlay 
approach it is common the assumption that PU message is known to the SU transmitter at the beginning of its transmission. While the latter assumption is impractical for an initial transmission, it holds for a message retransmission where the SU listen the first transmission and decodes it, in cases where the PU receiver cannot decode that message due to fading or interference. An alternative way is the PU to send its message to the SU (assuming that the corresponding channels are in good condition) prior to its transmission. Knowledge of a PU's message and/or codebook can be used to either cancel or mitigate the interference seen at the PU and SU users. This information can be used to completely cancel the interference generated by the PU signals at the SU receiver by e.g. dirty paper coding. Moreover, the SUs can use also this knowledge and allocate a part of their power for transmitting their data and the remaining part of their power to assist the PUs transmissions. By careful choice of the power allocated to each transmission, the PUs' rates remain unchanged while the SU allocates some power to his own transmissions. It is evident that the overlay approach is highly related to cooperative communication schemes that are discussed in detail in Section 1.5. It is noteworthy that the overlay approach can be applied to either licensed or unlicensed band communications. In licensed bands, the SUs are allowed to share the band with the licensed users, provided they improve the performance of the latter ones. In unlicensed bands, SUs would enable a higher spectral efficiency by exploiting message and codebook knowledge to reduce interference.

\subsubsection{The Interweave Approach}

The core idea behind the interweave approach is the opportunistic communication and was the original motivation for cognitive radio [1]. The idea emerged from the FCC [2] and industry studies showed that a major part of the spectrum in non utilized most of the time. That is, there are temporary unoccupied bands in the space-time-frequency domain commonly referenced as spectrum holes. The spectrum holes variate with respect to time and the geographic location and can be exploited by the SUs for their communication. Thus, the spectrum utilization is improved via the opportunistic use of the spectrum holes for the SUs' communication. An 
interweave technique requires knowledge of the PU activity in the spectrum. That is, the SU system must monitor the spectrum area of interest on a regular basis, detect the corresponding spectrum holes and then establish the SUs communication links. The so-called Spectrum Sensing techniques are usually employed for spectrum hole detection. Clearly the performance of an interweave technique is closely related to the performance of the employed spectrum sensing technique. Thus, the development

of efficient spectrum sensing techniques have been extensively studied in literature during the past years. In Section 1.4 we discuss in more detail the spectrum sensing problem. For more interesting details concerning the interweave approach we refer the reader to [3] and [12]-[14].

\subsubsection{Comparison of the Approaches}

In this subsection a comparative summary of the three cognitive radio approaches is given. The underlay and overlay techniques allow the SU transmissions to coexist with the PU ones, contrariwise to the interweave approach where the SU transmit only when there is no PU activity in the spectrum area of interest. It is noteworthy that the different approaches require different information at the SU side: In the underlay approach, the SUs require knowledge of the interference generated by the $\mathrm{SU}$ transmitter to the $\mathrm{PU}$ receiver. In the the overlay approach the $\mathrm{SU}$ require knowledge of the PUs codebook and possibly its message. Finally, in the interweave approach, knowledge of the PU activity is required which can been obtained by the spectrum sensing techniques as discussed in Subsection 1.3.3. Let us now consider the SUs' transmission power of each approach. In the overlay approach the SUs' power is limited only the corresponding device constraints. Contrariwise, in the underlay and interweave approaches, apart from the aforementioned device constraints, the transmission power is determined also by the interference constraint and the range of sensing, respectively. Before closing this section, it is worth noting that apart from the three distinct approaches, one may also propose hybrid versions that exhibit the advantages of the different ones [15]. 


\subsection{Spectrum Sensing Techniques}

Spectrum Sensing is by far the most significant component of the interweave techniques as it was stated in Subsection 1.3.3. It is defined as the task of obtaining awareness about the spectrum usage and existence of PUs in a geographical area. Traditionally, the spectrum sensing is considered as a method for measuring the spectral content, or measuring the radio frequency energy over the spectrum. In the CR terminology, it is a more general term that refers to the techniques that are employed so as to obtain the spectrum usage characteristics across the time, space, frequency and code dimensions. Furthermore, it involves the detection of the type of the signal that occupies currently the spectrum, including the waveform, the modulation, the carrier frequency, the bandwidth, etc. Nevertheless, this comes at the cost of increased computational complexity due to requirement of powerful signal analysis techniques.

Spectrum sensing may be implemented either by a single radio architecture or by a dual radio one. In the single radio case, which is the most common one, a fixed portion of each time slot is dedicated to spectrum sensing and the remaining part is used for data transmission. In the dual radio architecture, which has been recently proposed (see [16] and references therein), one radio chain is dedicated to data transmission and reception while a second radio chain is dedicated to continuous spectrum monitoring. The differences between the two approaches are discussed in [16]. In general, the double radio approach provides higher spectrum efficiency and better sensing accuracy, however it should employ very low-complexity spectrum sensing techniques due to its "continuous" nature.

Several spectrum sensing schemes have been proposed over the last few years including energy-based detectors (EBD), e.g. [17], eigenvalue - based spectrum sensing techniques (EBSS) [18]-[19], covariance - based spectrum sensing techniques [20], and cyclostationary - based spectrum sensing techniques [21]. The EBD methods are usually simple to implement, however, they require knowledge of the involved noise variance. The EBSS methods (particularly, the MMED and GLRT) may operate in a 
totally blind manner as they do not assume any knowledge concerning the PUs' signals or the noise variance, and also they may offer remarkably improved performance for specific signal categories.

The spectrum sensing techniques that were mentioned above refer to single-user

systems. Nevertheless, the performance of a single-user spectrum sensing technique is limited to environments where the fading and shadowing effects of the wireless channels degrade the quality of the received signals. Cooperation among multiple secondary users has been proposed in literature in order to improve the sensing performance. Note however, that the existing approaches (see [22]-[26] and references therein), are cooperative variations of the generic energy detector. To the best of our knowledge, cooperative techniques of the EBSS type have not been considered in literature so far, apart from the case where a fusion center collects the sensed data and applies the EBSS in a centralized batch manner [27]. A centralized approach comes with a number of limitations concerning the high power costs in transmitting local information to the fusion center and conveying global decisions back to the SUs. Moreover, a centralized SU network is quite sensitive to node and link failures. Contrariwise, a decentralized approach exhibits low communication overhead and it is robust to node and link failures. Each SU node communicates only with its adjacent SU nodes via one-hop transmissions resulting in reduced transmission power consumption during the sensing period. The SU nodes exchange information for several rounds so as to reach global convergence. Upon convergence, each node can reach the same decision concerning the PU existence without the need of a fusion center node. More information concerning the spectrum sensing techniques can be sought in [16], [28] and references therein.

\subsection{Cooperative Communication Techniques}

The initial motivation behind the cooperative communication techniques can be attributed to the groundbreaking work of Cover and El Gammal on the information theoretic properties of the relay channel [29]. In that paper, the capacity of a three 
node network consisting of a source, destination and relay node was analysed. Many of the ideas that appeared later in the cooperative communications' literature were first exposited in [29]. Nevertheless, the cooperative communication that is considered in the recent literature is somewhat different from the relay channel of [29]. In general, there are three types of nodes in the cooperative communications' terminology. The (S)ource, the (D)estination and the (R)elay(s) nodes, as it was already mentioned. The aim is to transmit information from the source to the destination node via employing the relay network in order to improve the quality of the overall transmission. The relay(s) simply forward(s) the information of the source node to the destination node without actually being an information source or sink. Conceptually, information is relayed in two phases or modes: In the first phase, the source node transmits and the relay(s) and destination nodes receive, which is commonly referenced as the broadcast phase; In the second phase, the source and relay(s) nodes transmit and the destination one receives. This phase is commonly known as the multiple-access one. It is noteworthy that the latter differentiation is only conceptual since simultaneous communication is possible in both phases. The following different models of relaying can be classified based on the above two phases.

1. $S \rightarrow(R, D) ;(S, R) \rightarrow D$ (The most general form);

2. $S \rightarrow R ;(S, R) \rightarrow D$ (D ignores the signal of $\mathrm{S}$ node in the first phase);

3. $S \rightarrow(R, D) ; R \rightarrow D$ (S does not transmit in the second phase);

4. $S \rightarrow R ; R \rightarrow D$ (Multi-hop communication).

The first model is the most general one and it was employed by the majority of the early works in the are of cooperative communications. The second and the third model are simplified models that were introduced mainly for analytical tractability. For example, they simplify the outage probability analysis and the design of the spacetime codes for fading relay channels [30]- [36]. The last model is much older as well as simpler than the other three and is commonly referenced as multi-hop communication. Contrariwise to the other three models, the multi-hop communication approach does 
not yields diversity benefits and is in general employed to combat signal attenuation in long-range communication. For example in wireless systems which suffer from severe signal attenuation in long-range communications, if the targeted destination node is placed in such a distance from the source node that direct communication is (almost) impossible, a chain of relay nodes may be employed in order to establish the desired communication link via short-range multi-hop transmissions. A characteristic of multi-hopping is that each node in the chain communicates only with the one before and the one after in the chain. It is interesting to note that, of all the modes of user cooperation, multi-hopping is the only one that is widely implemented today.

Relaying strategies can be discriminated according to the relay duplexing mode. That is, whether it involves half-duplex or full-duplex relay nodes. A relay node is characterized as a half-duplex one, if it cannot simultaneously transmit and receive data in the same band. In other words, the transmission and reception channels must be orthogonal. The latter orthogonality can be achieved in either in time domain or in frequency domain or by employing sets of signals that are orthogonal over the timefrequency plane. A simultaneous transmission in the same frequency band results in interference generation to the received signal. The interference cancellation is not a trivial task that makes the implementation of full-duplex techniques challenging. A number of approaches have been proposed so far in the literature of full-duplex relays that are built around different methods for cancelling the so to speak interference [37][40]. It is interesting though to note, that the early information theoretic literature was based on full-duplex relaying [29] whereas the majority of the recent advances in the field is based on half-duplex protocols [30]-[36] and [41]-[42].

In general, there are two fundamental approaches based on which the source and relay nodes share their resources to achieve the highest throughput possible for any known coding scheme. The cooperation strategies based on these different ideas have commonly referenced as relay protocols.

The first protocol involves decoding of the source transmissions at the relay. Then, the relay retransmits the decoded signal after possibly compressing or adding redundancy. This protocol is known as the Decode-and-Forward one, for obvious reasons. 
The DF protocol achieves performance close to the optimal one when the source-relay channel is in relative good condition, which practically happens when the source and the relay nodes are physically near to each other.

The second approach is important when the source-relay and the source-destination channels are comparable, and the relay destination link is good. If this is the case, the relay may not be able to decode successfully the source signal, though it has an independent observation of the source signal that can aid the destination in the decoding procedure. Thus, the relay sends its estimate of the source transmission to the destination. This approach is known as the estimate-and-forward (also known as compress-and-forward or quantize-and-forward) protocol.

A special case of the above strategy is the so-called Amplify-and-Forward (AF) protocol where the estimate of the source transmission is simply the signal received by the relay, scaled up or down before retransmission. The amplification factor is dictated by the relative strengths of the source-relay and source-destination links $[30]$.

In the past years, the field of cooperative communications has been extensively researched and numerous cooperative based communication techniques have been developed. Multi-antenna relay techniques were developed for either the half- or the full-duplex case [43]-[45]. Relay-assisted cognitive radio techniques have been proposed where the secondary users act as relays to the primary user in order to assist the latter's communication [46]-[49]. Beamforming techniques have been also developed for cooperative systems [50]-[54] and cooperative cognitive systems [55]-[57]. The distributed nature of the cooperative systems, the individual power constraints of each relay node and the possible half-duplex constraint are in general the reasons that make challenging the development of an efficient cooperative communication scheme. For example problems that are well-studied in literature such as channel estimation, beamforming weight computation e.t.c., may prove to be very challenging and hard to solve when cooperative systems are considered. Therefore, a part of this dissertation has been devoted to the study of two specific problems that are raised in the cooperative systems literature and corresponding solutions are proposed. 


\subsection{Distributed Estimation}

Over the past years, a large number of adaptive estimation techniques have been developed for communication systems in literature, such as adaptive channel estimation/equalization schemes, adaptive beamforming schemes e.t.c. In several cases, as for example when the involved channels are time-varying, the employment of an adaptive scheme provides significant performance improvement compared to one of the corresponding batch scheme. Nevertheless, the distributed nature of the cooperative systems render challenging the application of adaptive schemes and a significant part of the recent signal processing literature deals with the matter.

Clearly, on the most significant parts in a distributed processing scheme is the way the cooperating nodes interact so as to estimate the require quantity. The previous "interaction" term refers to the type of the information that is interchanged among the nodes (analogue or digital) and the way the latter information passes around at the nodes (which nodes communicate and with what strategy, i.e. is a fusion center assumed or a decentralized approach is considered). In general, the way the information is circulated among the network nodes is dictated by the corresponding network topology. Large number of the published works deal with completely decentralized solutions that can be discriminated in two major categories: the incremental based strategies and the diffusion - consensus based strategies.

In the incremental approach, the information is interchanged only between adjacent nodes in a sequential manner. This mode requires a cyclic pattern of collaboration among the nodes and tends to require the least amount of communication overhead and power [58]-[61]. On the contrary, in the diffusion [62]-[64] and the consensus [65]-[68] approaches, each of the nodes communicates with all its neighbours. As a result, the communication overhead in these cases is higher than in an incremental one. Nevertheless, each node have obviously access to more data from the adjacent ones. The communication overhead in the diffusion / consensus approaches can be reduced by permitting each node to communicate with only a subset of the its adjacent ones. The choice of the subset can be randomized according to some 
performance criterion. A detailed discussion regarding the differences between the diffusion and consensus approaches is beyond the scopes of the present dissertation. A comparative study can be found in [69].

\subsection{Contributions and Outline}

In this chapter, the motivation and the contributions of the dissertation are summarized.

The first contribution is a novel interweave communication scheme for multiantenna CR systems. The majority of the interweave approach literature concern techniques that exploit frequency or time domain spectrum holes. Multi-antenna systems provide spectrum holes on the spatial domain that can be exploited by SUs in the CR context. To the best of our knowledge, the majority of the existing techniques were developed assuming perfect Channel State Information (CSI) knowledge. In a more realistic approach one should examine also an estimation scheme for the aforementioned CSI and examine its impact on the performance of the technique. In fact, the amount of CSI that a technique requires could render it impractical. For example, according to Section 1.3.3, it is evident that in the interweave approach, the PUs desire the minimum possible interaction with the corresponding SUs. That is, if the technique to be applied requires full CSI (that is, exact knowledge of the fading coefficients) of the PU-SU cross channels, a training based estimation scheme must be employed. The aforementioned scheme requires the $\mathrm{SU}$ to have access to the $\mathrm{PU}$ training symbols and moreover, to be somehow synchronized to its training symbol periods. The previous of course, contradicts with the interweave approach philosophy. Therefore, at first we propose a novel interweave scheme that requires CSI that can be estimated in a completely blind manner. Then, the corresponding blind estimation scheme is developed. Another significant contribution is the theoretical results that were derived for both the perfect CSI case and the imperfect CSI case (that is when the blind estimation scheme is employed for obtaining the corresponding CSI). Especially the theoretical results that concern the imperfect CSI case is one of the 
first ones that appear in the relevant literature, to the best of our knowledge. The previous results are presented in Chapter 2 and in [70]-[71].

The second contribution is a decentralized adaptive eigenvalue-based spectrum sensing technique for multi-antenna CR Systems. As it was presented in Section 1.4, spectrum sensing is a fundamental functionality in CR systems that are based on the interweave approach. Eigenvalue-Based Spectrum Sensing (EBSS) techniques are known to achieve good performance and also to be applicable in a completely blind manner. In the literature so far, only batch and centralized cooperative EBBS techniques have been considered which, however, suffer from limitations that render them impractical in several cases such as, when time-varying channels are involved or when continuous spectrum monitoring is required (dual-radio) architectures. Thus, the aim here is to develop practical cooperative adaptive versions of typical EigenvalueBased Spectrum Sensing (EBBS) techniques which could be applied in a completely decentralized manner and cope well in time-varying scenarios. To this end, at first, novel adaptive EBSS techniques are developed for a single-user (no cooperation) case. Then, a novel distributed subspace tracking method is proposed which enables the cooperating nodes to track the joint subspace of their received signals. Based on this method, cooperative decentralized versions of the adaptive EBSS techniques are subsequently developed that overcome the limitations of the existing batch centralized approaches. The results are presented in Chapter 3 and in [72]-[73].

The third contribution is a new cooperative scheme for half-duplex uplink transmission. Half-duplex relaying schemes suffer from multiplexing gain loss due to the two phase transmission they require. In literature so far, there are several approaches that address the issue though they present limitations such as the loss of the orthogonality of the two-phase transmissions resulting in interference generation (for a detailed survey please check Section 3.1). In this dissertation a novel technique is developed based on a virtual MIMO structure formed by the single antenna source and relays nodes along with the multi-antenna base station which is the destination node. The new technique aims at providing the opportunity to the cooperative system to exhibit increased diversity gains without suffering the multiplexing gain loss that is 
common in the majority of the existing cooperative techniques. The theoretical outage probability and the corresponding Diversity Multiplexing Trade-off (DMT) curve of the proposed technique are derived so as to verify its performance. The latter results are presented in Chapter 4 and in [74]- [76].

The final contribution is a set of two novel algorithms which enable the relay cooperation for the distributed computation of the beamforming weights in a blind and adaptive manner, without the need to forward the data to a fusion center. Cooperative beamforming schemes consist a significant part in cooperative systems' and CR cooperative systems' literature. While the computation of the beamforming weights is quite simple under perfect CSI, it becomes very challenging when the required CSI must be estimated due to the distributed nature of the cooperative systems. Therefore, we were motivated to develop of a decentralized estimation scheme for the computation of the weights of known cooperative beamforming schemes. The proposed algorithms are constituent parts of two corresponding distributed beamforming schemes for relay networks that distribute the computational overhead equally among the relay nodes. In the first scheme, the beamforming vector is computed through minimization of the total transmit power subject to a receiver quality-of-service constraint (QoS). In the second scheme, the beamforming weights are obtained through maximization of the receiver signal-to-noise-ration (SNR) subject to a total transmit power constraint. The proposed approaches achieve close performance to the one of the optimal beamforming solutions derived assuming perfect channel state information at the relays' side. The previous results are presented in Chapter 5 and in $[77]$.

Finally, in Chapter 6, general conclusions are drawn and open research issues are discussed.

\subsection{Conclusion}

In this chapter, a brief survey of literature topics relevant to the ones studied in this dissertation was given along with a presentation of our contributions to the 
field. In the beginning the basic characteristics of the cognitive radio systems were described. Then, a more detailed reference to the spectrum sensing techniques was given. After that, the key features of the cooperative systems where presented. After that, a brief survey of the adaptive distributed estimation literature was given. Then, the contributions and the outline of the dissertation where presented. Finally the contributions and the outline of the dissertation was given We can now proceed to the description of the problems considered in the present dissertation. 


\section{Chapter 2}

\section{Blind Opportunistic Interference Alignment in MIMO Cognitive Radio Systems}

\section{$2.1 \quad$ Introduction}

As it was discussed in Section 1.3 the first techniques developed in the CR context were based on the interweave approach. Initially, interweave CR techniques that were proposed in literature were concerned with the exploitation of spectral holes that may exist either in the time or in the frequency domain. Subsequently, it was suggested that multiple antenna wireless systems may provide exploitable degrees of freedom in the spatial domain since it is possible that some of them are not employed by the corresponding PUs. Proper exploitation of these spatial holes may be achieved by applying the so-called Interference Alignment (IA) concept [78]-[79]. Generally speaking, an IA technique aligns the interference signals to a subspace orthogonal to the one(s) of the signals of interest at the intended receiver. A common characteristic of most of the IA techniques is, that some kind of cooperation [79]-[80] between the nodes of the network is assumed. Such an assumption may be appropriate for networks of nodes with equal access rights to the spectrum area of interest but it 
may be not valid for typical interweave CR scenarios. Typically, in CR networks employing interweave schemes, the PUs should operate without any interaction with the SUs. Thus, it is of interest to design IA techniques in which the alignment of the signals to orthogonal subspaces is handled completely by the SUs without degrading the PU system's performance.

Motivated by the IA concept, the authors in [81] proposed an Opportunistic Interference Alignment (OIA) technique appropriate for Multiple-Input Multiple-Output (MIMO) CR networks, that is, to CR networks with multi-antenna nodes. In their approach, the PU applies the Water-Filling (WF) algorithm to optimize its transmission through the corresponding singular channels [82]. It is, however, possible that the WF algorithm allocates zero power to some of the singular channels due to their bad condition. That is, there are free spatial degrees of freedom that can be opportunistically exploited by the SU. Thus, the SU aligns its signals to these free degrees of freedom by appropriately pre-coding the data to be transmitted.

It should be noted that, most of the existing IA / OIA techniques [78]-[80] have been developed by considering perfect CSI at the SUs. Therefore, it is of great importance to develop practical CSI estimation schemes and study their performance so as to gain insight into the applicability of the IA techniques. Recently, a number of works appeared in the literature which study the performance of IA techniques under imperfect CSI conditions, e.g., when CSI is estimated by training-based techniques (see [83]-[84] and references therein). Note, that a training-based approach assumes knowledge of the PU training-symbols at the SU receiver. However, in a CR network, it would be highly desirable for the SU to have the minimum possible interaction with the PU and, moreover, the PU network to completely ignore the SU one. A possible means for eliminating the need of training symbols transmission (known also to the SUs) would be to estimate the CSI required to the SU in a blind manner.

However, the technique in [81] is not suitable for blind estimation of the required CSI since the SU post-coding matrix cannot be estimated without training symbols send by the PU. Moreover, in [81], the SU deals exclusively with the alignment of the PU and SU signals to orthogonal subspaces at the PU receiver only, whereas, the 
alignment of the signals at the SU receiver is not considered at all. Thus, the received SU signals are always corrupted by the PU transmissions and noticeable performance loss may be observed in several cases. The above observations motivated us to seek for an OIA technique in which a) two different IA constraints are satisfied, one for the PU and one for the SU link and b) all the required CSI can be estimated in a blind manner requiring zero interaction with the corresponding PU. That is, appropriate pre- and post-coding matrices are derived so as to align the signals to orthogonal subspaces at the PU and SU receivers simultaneously.

Let us now summarize the contributions. At first, a new OIA method is developed that is based on a similar method proposed in [81]. In the proposed approach [70]-[71] the PU communication technique is based on a SVD parallel channel decomposition scheme that employs the space-time version of the Water-Filling (WF) Algorithm for power allocation whereas in [81] the space-only WF algorithm was used. As it is shown, the space-time version of the WF algorithm provides the opportunity to the SU to achieve a slightly improved performance and is also more tractable to theoretical analysis. In addition, the proposed SU communication scheme is designed so as to satisfy two different IA constraints, as described above. The performance of the proposed method is studied theoretically and close approximations of the ergodic capacity of the PU and the SU systems are derived. As it is proved, the proposed approach achieves significantly better performance for SU systems with larger number of antennas than the corresponding PU systems. Moreover, a major advantage of the proposed version over the original one, is that the CSI required at the SU system can be blindly estimated from the second order statistics of the received signals. To this end, a novel blind estimation scheme has been developed for both of the SU ends. The impact of the aforementioned blind estimation technique on the performance of the proposed OIA scheme is investigated and some theoretical results are also derived. It turns out that the proposed blind scheme, even with few received samples, may provide very accurate estimates of the pre- and post-coding matrices that align the SU signals to orthogonal subspaces to the PU ones. The rest of the chapter is organized as follows. In Section 2.2, the system description is provided. In Section 


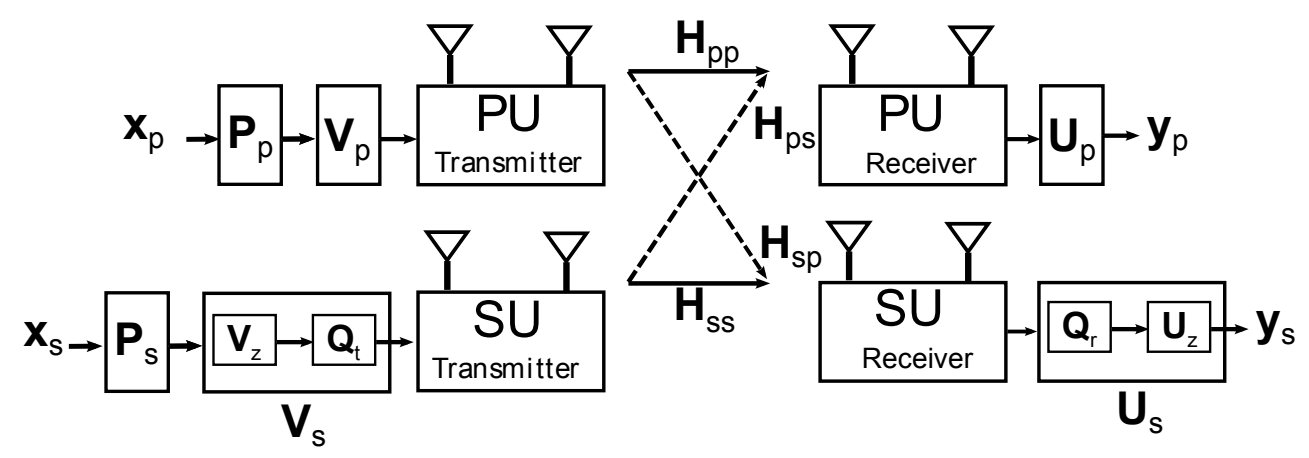

Figure 2-1: System Model

2.3, the original OIA approach of [81] and the proposed extension are presented. In Section 2.4, a novel technique that enables the SU to blindly estimate the required information is derived. Section 2.5 provides the performance analysis of the proposed technique. In Section 2.6, some representative simulations are presented and Section 2.7 concludes the chapter.

\subsection{System Description}

Let us assume that a $T_{p} \times R_{p}$ MIMO PU and a $T_{s} \times R_{s}$ MIMO SU are operating in the same frequency band (Fig.2-1). Each user employs a pair of pre-coding and postcoding matrices, denoted as $\mathbf{V}_{p}, \mathbf{U}_{p}$ and $\mathbf{V}_{s}, \mathbf{U}_{s}$ for the $\mathrm{PU}$ and the SU, respectively. The input-output relationship is given by

$$
\left[\begin{array}{c}
\mathbf{y}_{p} \\
\mathbf{y}_{s}
\end{array}\right]=\left[\begin{array}{c}
\mathbf{U}_{p}^{H} \\
\mathbf{U}_{s}^{H}
\end{array}\right]\left(\left[\begin{array}{ll}
\mathbf{H}_{p p} & \mathbf{H}_{p s} \\
\mathbf{H}_{s p} & \mathbf{H}_{s s}
\end{array}\right]\left[\begin{array}{c}
\mathbf{V}_{p} \mathbf{P}_{p} \mathbf{x}_{p} \\
\mathbf{V}_{s} \mathbf{P}_{s} \mathbf{x}_{s}
\end{array}\right]+\left[\begin{array}{c}
\mathbf{z}_{p} \\
\mathbf{z}_{s}
\end{array}\right]\right)
$$

where matrices $\mathbf{H}_{p p}, \mathbf{H}_{s s}, \mathbf{H}_{p s}$ and $\mathbf{H}_{s p}$ denote the flat fading channels between the $\mathrm{PU}$ transmitter and the $\mathrm{PU}$ receiver, the $\mathrm{SU}$ transmitter and the $\mathrm{SU}$ receiver, the SU transmitter and the PU receiver, and the PU transmitter and the SU receiver, respectively, $\mathbf{P}_{p}$ and $\mathbf{P}_{s}$ are the $\mathrm{PU}$ and $\mathrm{SU}$ diagonal power allocation matrices, $\mathbf{x}_{p}$ and $\mathbf{x}_{s}$ are the vectors of the symbols transmitted by PU and SU, respectively, and they are i.i.d. zero mean random variables, i.e. $\mathbb{E}\left\{\mathbf{x}_{p} \mathbf{x}_{p}^{H}\right\}=\mathbf{I}_{T_{p}}$ and $\mathbb{E}\left\{\mathbf{x}_{s} \mathbf{x}_{s}^{H}\right\}=\mathbf{I}_{T_{s}}$,

where $\mathbb{E}\{\cdot\}$ denotes the expected value operator, $\mathbf{y}_{p}$ and $\mathbf{y}_{s}$ are the received $\mathrm{PU}$ and 
$\mathrm{SU}$ vectors and $\mathbf{z}_{p}$ and $\mathbf{z}_{s}$ are vectors of Additive White Gaussian Noise (AWGN) samples of zero mean and variance $\sigma_{n}^{2}$. The transmissions are subject to the following power constraints

$$
\begin{aligned}
& \operatorname{tr}\left(\mathbf{V}_{p} \mathbf{P}_{p}^{2} \mathbf{V}_{p}^{H}\right) \leq P_{p} \\
& \operatorname{tr}\left(\mathbf{V}_{s} \mathbf{P}_{s}^{2} \mathbf{V}_{s}^{H}\right) \leq P_{s}
\end{aligned}
$$

where $\operatorname{tr}(\mathbf{A})$ is the trace and $\mathbf{A}^{H}$ the Hermitian of matrix $\mathbf{A}$.

\subsection{Opportunistic Interference Alignment}

In the OIA techniques, pre/post-coding matrices are properly designed so that the SU transmission is aligned to the PU's unexploited degrees of freedom and is also optimized without degrading the performance of the latter. In this section, first, the PU and SU transmission schemes of [81] are briefly described and then, the proposed approach is presented. Note that from now on, the abbreviation SU refers to the whole SU link, which includes both the SU transmitter and the SU receiver (and similarly for the PU) unless it is otherwise explicitly stated.

\subsubsection{The Opportunistic Scheme of the Original Approach}

According to the IA concept, the SU transmissions should not degrade the PU ones. Therefore, the PU's pre- and post-coding matrices are designed as if there were no interfering signals. Thus, a capacity achieving parallel channel decomposition scheme is applied [82] in which the involved pre- and post-coding matrices are computed by the Singular Value Decomposition (SVD) of $\mathbf{H}_{p p}$ given by $\mathbf{H}_{p p}=\mathbf{U}_{p} \boldsymbol{\Sigma}_{p} \mathbf{V}_{p}^{H}$, where $\mathbf{V}_{p}$ and $\mathbf{U}_{p}$ are the $T_{p} \times T_{p}$ and $R_{p} \times R_{p}$ singular vectors' matrices and $\Sigma_{p}$ is a $R_{p} \times T_{p}$ diagonal matrix containing the singular values. Therefore, the data are transmitted via the parallel independent channels as

$$
\mathbf{y}_{p}=\Sigma_{p} \mathbf{P}_{p} \mathbf{x}_{p}+\tilde{\mathbf{z}}_{p}
$$


where $\tilde{\mathbf{z}}_{p}=\mathbf{U}_{p}^{H} \mathbf{z}_{p}$ is an AWGN vector, due to the unitary structure of $\mathbf{U}_{p}$. Matrix $\mathbf{P}_{p}$ is computed by applying the so-called Water-filling (WF) algorithm [82]. That is, the power $p_{p}^{(l)}$ allocated to the channel associated to the $l-t h$ singular value $\sigma_{p_{l}}$ of matrix $\mathbf{H}_{p p}$ is given by

$$
p_{p}^{(l)}=\left(\beta_{p}-\sigma_{n}^{2} / \lambda_{l}\right)^{+}, 1 \leq l \leq \min \left(T_{p}, R_{p}\right)
$$

where, $(\cdot)^{+}=\max \{\cdot, 0\}, \lambda_{l}=\sigma_{p_{l}}^{2}$ and the water-level $\beta_{p}$ is computed so that the power constraint of (2.2) is satisfied. Due to the involved WF algorithm, those channels which are relatively bad are not employed by the PU system. Thus, the main objective of an OIA technique is to align the SU transmissions to these free channels, so that zero interference is generated to the PUs.

Let us now proceed with the description of the SU transmission scheme. Each one of the SU pre- and post-coding matrices is expressed as a product of two orthonormal matrices, that is, $\mathbf{V}_{s}=\mathbf{Q}_{t} \mathbf{V}_{z}$ and $\mathbf{U}_{s}=\mathbf{Q}_{r} \mathbf{U}_{z}$, respectively (Fig. 2-1). Matrices $\mathbf{Q}_{r}$ and $\mathbf{Q}_{t}$ are used in order the interference constraints to be imposed, while matrices $\mathbf{U}_{z}$ and $\mathbf{V}_{z}$ are applied so as the $\mathrm{SU}$ transmission to be optimized by diagonalizing the equivalent channel matrix. More specifically, in order to satisfy the zero interference constraint to the PU receiver (IA condition), the SU transmitter pre-codes the transmitted data by a matrix $\mathbf{Q}_{t}$. Then, according to eq.(2.1) the received signal at the PU receiver is written as

$$
\mathbf{y}_{p}=\underbrace{\boldsymbol{\Sigma}_{p} \mathbf{P}_{p} \mathbf{x}_{p}}_{\text {signal }}+\underbrace{\mathbf{U}_{p}^{H} \mathbf{H}_{p s} \mathbf{Q}_{t} \mathbf{V}_{z} \mathbf{P}_{s} \mathbf{x}_{s}}_{\text {interference }}+\tilde{\mathbf{z}}_{p},
$$

If $L_{p} \leq \min \left\{T_{p}, R_{p}\right\}$ is the number of the employed PU channels, the interference term of eq.(2.5) is nulled-out if

$$
\mathbf{Q}_{t} \in \mathcal{N} \mathcal{U} \mathcal{L} \mathcal{L}\left\{\mathbf{U}_{p}^{H}\left(1: R_{p}, 1: L_{p}\right) \mathbf{H}_{p s}\right\}
$$

where $\mathcal{N} \mathcal{U L} \mathcal{L}\{\mathbf{A}\}$ denotes the null space of matrix $\mathbf{A}$. Assuming now that $\mathbf{Q}_{t}$ satisfies 
eq.(2.6), the next goal is to optimize the SU transmissions. At first, the received data are post-coded by a matrix $\mathbf{Q}_{r}$ so that the received SINR is maximized. The latter matrix is given by [81]

$$
\mathbf{Q}_{r}=\left(\mathbf{H}_{s p} \mathbf{V}_{p} \mathbf{P}_{p}^{2} \mathbf{V}_{p}^{H} \mathbf{H}_{s p}^{H}+\sigma_{n}^{2} \mathbf{I}_{R_{s}}\right)^{-1 / 2}
$$

Thus, according to (2.6) and (2.7) the $\mathrm{SU}$ operates as an equivalent $\left(T_{s}-L_{p}\right) \times R_{s}$ MIMO system with channel matrix $\mathbf{Z}=\mathbf{Q}_{r}^{H} \mathbf{H}_{s s} \mathbf{Q}_{t}$. If $\mathbf{Z}=\mathbf{U}_{z} \boldsymbol{\Sigma}_{z} \mathbf{V}_{z}^{H}$, the $\mathrm{SU}$ can use as pre- and post-coding matrices the $\mathbf{V}_{s}=\mathbf{Q}_{t} \mathbf{V}_{z}$ and $\mathbf{U}_{s}=\mathbf{Q}_{r} \mathbf{U}_{z}$ ones, respectively, and apply a parallel channel decomposition scheme, similar to the one described in the present subsection for the PU system.

Remark 2.1 One may consider the above technique more as an opportunistic zeroforcing beamformer rather than an IA technique. However, since our technique was motivated by the work in [81], which was termed as an IA one, we prefer to keep the same terminology in order to avoid possible confusion.

Remark 2.2 The OIA technique can be applied as long as $T_{s}>L_{p}$. If the latter inequality does not hold, then the required null-space has zero dimensions and the $S U$ remains silent during these timeslots. Of course if $T_{s}>\min \left\{T_{p}, R_{p}\right\}$ there is always a non-zero null-space of at least $\left(T_{s}-\min \left\{T_{p}, R_{p}\right\}\right) \times R_{s}$ dimensions.

Remark 2.3 A direct way to compute matrices $\mathbf{Q}_{t}$ and $\mathbf{Q}_{r}$ would require CSI (which is available to the PU nodes) to be provided to the $S U$ nodes as well. To avoid such a demanding interaction between the two networks we seek here a blind scheme for estimating these matrices. It has already been mentioned that matrix $\mathbf{Q}_{t}$ is a nullspace basis of $\mathbf{U}_{p}^{H}\left(1: R_{p}, 1: L_{p}\right) \mathbf{H}_{p s}$. Due to this fact, as shown in Section 2.4, it is possible to estimate this matrix blindly from the covariance matrix of the received signal at the SU transmitter. On the contrary, a blind estimation scheme for $\mathbf{Q}_{r}$ would not be possible, since full knowledge of matrices $\mathbf{H}_{s p}, \mathbf{V}_{p}$ and $\mathbf{P}_{p}$ is required according to eq.(2.7). To circumvent this problem, we propose in the subsection III.B an alternative approach to compute $\mathbf{Q}_{r}$. 


\subsubsection{The Proposed Approach}

Let us first consider the case of the PU scheme whose basic structure will be the same with the original one described in Subsection 2.3.1. That is, a SVD-based parallel channel decomposition is applied and the input-output relationship of the system is given by eq.(2.3). The only difference in the PU system is on the computation of the diagonal power allocation matrix $\mathbf{P}_{p}$ which is now obtained by applying the socalled space-time version of the WF algorithm [85] instead of the spatial-only version considered in [81]. The space-time WF algorithm is more tractable to theoretical analysis and, as shown in the simulations section, it may offer a sightly improved SU performance for the same PU performance. A comparative study between the space-time and the space-only approach for general MIMO systems can be found in [86].

In order to apply the space-time WF algorithm, the instantaneous constraints of eq.(2.2) are replaced by mean ones. That is, the PU and $\mathrm{SU}$ transmissions are subject to

$$
\begin{aligned}
& \mathbb{E}\left\{\operatorname{tr}\left(\mathbf{V}_{p} \mathbf{P}_{p}^{2}\left(\boldsymbol{\Sigma}_{p}\right) \mathbf{V}_{p}^{H}\right)\right\} \leq \bar{P}_{p} \\
& \mathbb{E}\left\{\operatorname{tr}\left(\mathbf{V}_{s} \mathbf{P}_{s}^{2}\left(\boldsymbol{\Sigma}_{z}\right) \mathbf{V}_{s}^{H}\right)\right\} \leq \bar{P}_{s}
\end{aligned}
$$

where $\boldsymbol{\Sigma}_{z}$ is the diagonal matrix of the singular values of the equivalent SU channel matrix $\mathbf{Z}$ defined later in this section. Thus, the power $p_{p}^{(i)}$, allocated to the channel associated to the $i-t h$ singular value $\sigma_{p_{l}}$ of matrix $\mathbf{H}_{p p}$, is given by

$$
p_{p}^{(l)}=\left(\bar{\beta}_{p}-\sigma_{n}^{2} / \lambda_{l}\right)^{+}, 1 \leq l \leq \min \left(T_{p}, R_{p}\right)
$$

where now the mean water-level $\bar{\beta}_{p}$ is computed by numerically solving eq.(2.10) so as the PU power constraint of eq.(2.8) is satisfied

$$
\sum_{l=1}^{L_{p}} \int_{\sigma_{n}^{2} / \bar{\beta}_{p}}^{\infty}\left(\bar{\beta}_{p}-\frac{\sigma_{n}^{2}}{\lambda_{l}}\right) f\left(\lambda_{l}\right) d \lambda_{l}=
$$




$$
\sum_{l=1}^{L_{p}} \frac{\bar{\beta}_{p} \bar{\lambda}_{l} \Gamma\left(K_{l}, \frac{K_{l} \sigma_{n}^{2}}{\bar{\beta}_{p} \bar{\lambda}_{l}}\right)-K_{l} \sigma_{n}^{2} \Gamma\left(K_{l}-1, \frac{K_{l} \sigma_{n}^{2}}{\bar{\beta}_{p} \lambda_{l}}\right)}{\bar{\lambda}_{l}\left(K_{l}-1\right) !}=\bar{P}_{p}
$$

where $f\left(\lambda_{l}\right)$ is the marginal probability density function (pdf) of the random variable (rv) $\lambda_{l}, \bar{\lambda}_{l}=\mathbb{E}\left\{\lambda_{l}\right\}, L_{p}=\min \left\{T_{p}, R_{p}\right\}, K_{l}=\left(T_{p}-l+1\right)\left(R_{p}-l+1\right)$ and $\Gamma(\cdot, \cdot)$ is the upper incomplete gamma function [87].

Let us now proceed with the description of the proposed SU transmission scheme. The main goal of the proposed approach is to derive appropriate pre- and post-coding $\mathrm{SU}$ matrices which align the $\mathrm{SU}$ and $\mathrm{PU}$ signals to orthogonal subspaces at both the PU and SU receivers. That is, an additional IA constraint should be satisfied as opposed to the original approach of [81], where the pre-coding matrix of the $\mathrm{SU}$ $\mathrm{Q}_{t}$ (eq.(2.6)) was used to achieve the aforementioned orthogonality only at the PU receiver. Since the pre-coding matrix of eq.(2.6) satisfies the IA condition at the PU receiver, we propose an alternative approach for the design of the SU receiver's post-coding matrix $\mathbf{Q}_{r}$ so as to satisfy the second IA constraint.

Let us assume that the SU transmitter pre-codes the transmitted data by using $\mathbf{Q}_{t}$ only. According to the system description in eq.(2.1), the received signal at the $\mathrm{SU}$ receiver is

$$
\mathbf{y}_{s}=\underbrace{\mathbf{H}_{s s} \mathbf{Q}_{t} \mathbf{P}_{s} \mathbf{x}_{s}}_{\text {signal }}+\underbrace{\mathbf{H}_{s p} \mathbf{V}_{p} \mathbf{P}_{p} \mathbf{x}_{p}}_{\text {interference }}+\mathbf{z}_{s} .
$$

Similarly to matrix $\mathbf{Q}_{t}$ derivation, the interference term of eq.(2.11) is nulled-out if $\mathbf{Q}_{r}^{H} \mathbf{H}_{s p} \mathbf{V}_{p}=\mathbf{0}_{R_{s} \times T_{p}}$. Therefore matrix $\mathbf{Q}_{r}$ must satisfy,

$$
\mathbf{Q}_{r} \in \mathcal{N} \mathcal{U} \mathcal{L} \mathcal{L}\left\{\mathbf{H}_{s p} \mathbf{V}_{p}\left(1: T_{p}, 1: L_{p}\right)\right\}
$$

Thus, the symbols $\mathbf{x}_{s}$ are transmitted through an equivalent $\left(T_{s}-L_{p}\right) \times\left(R_{s}-L_{p}\right)$ MIMO system with channel matrix $\mathbf{Z}=\mathbf{Q}_{r}^{H} \mathbf{H}_{s s} \mathbf{Q}_{t}$ and a SVD-based parallel channel decomposition can be applied as described in Subsection 2.3.1 for the original SU approach.

Remark 2.4 A common approach in the literature is to use in eq.(2.10) the empirical 
pdf $f(\lambda)$ of the unordered eigenvalues ([85]-[86]). However, in the present work, we have adopted using the close approximations of the ordered marginal eigenvalue pdfs derived in [88]. The latter approach enables us to derive the second part of eq.(2.10) which is applicable to any MIMO system with an arbitrary number of transmitter and receiver antennas. To the best of our knowledge, a similar general result cannot be derived in the case of the empirical eigenvalue pdf, mainly due to its complex structure [85]. It is noteworthy that the proposed approach has identical performance with the one based on the empirical eigenvalue pdf.

Remark 2.5 As shown in Section 2.4, the proposed approach enables the SU to use a completely blind procedure to estimate the required CSI information. However, it exhibits an extra loss of $L_{p}$ degrees of freedom at the $S U$ receiver due to the interference nulling procedure. Thus, a degradation in the SU performance is expected for systems where $\min \left\{T_{s}, R_{s}\right\}$ is close to $\min \left\{T_{p}, R_{p}\right\}$. On the contrary, in the proposed approach, the interference caused by the PU transmission at the $S U$ receiver is completely nulled-out and the equivalent system matrix $\mathbf{Z}=\mathbf{Q}_{r}^{H} \mathbf{H}_{s s} \mathbf{Q}_{t}$ shares the same statistical properties with $\mathbf{H}_{s s}$ due to the orthonormal properties of matrices $\mathbf{Q}_{r}$ and $\mathrm{Q}_{t}$. Therefore, the proposed approach is expected to achieve better performance as long as $\min \left\{T_{s}, R_{s}\right\}$ is not close to $\min \left\{T_{p}, R_{p}\right\}$.

\subsection{Blind Opportunistic Interference Alignment}

In this section a novel scheme is developed that enables the $\mathrm{SU}$ to estimate the required CSI information in a blind manner. According to Section 2.3, the SU requires knowledge of $\mathbf{Q}_{t}$ and $\mathbf{V}_{z}$ at its transmitter side, and knowledge of $\mathbf{Q}_{r}$ and $\mathbf{U}_{z}$ at its receiver side. The challenging task here is to design an estimation scheme for matrices $\mathrm{Q}_{r}$ and $\mathbf{Q}_{t}$ which contain information concerning the $\mathrm{PU}$ transmission. Recall that most of the IA techniques assume that the SU has somehow access to information concerning the PU transmission via, for example, a PU-to-SU feedback mechanism.

At first, a blind estimation technique is proposed for matrices $\mathbf{Q}_{r}$ and $\mathbf{Q}_{t}$ by observing that the latter matrices can be derived via the null-space of the received 
signals' covariance matrix at the SU receiver and transmitter, respectively. Then, it is shown that a similar blind scheme can also be derived for estimating $\mathbf{V}_{z}$ and $\mathbf{U}_{z}$. The complete estimation procedure is summarized at Table 2-I.

Let us consider that the PU system uses a Time Division Duplex (TDD) mechanism and hence channel reciprocity may be assumed between the forward (transmitter to receiver) and the reverse (receiver to transmitter) channels. The PU receiver periodically transmits feedback or data to the transmitter through the reverse channel $\mathbf{H}_{p p}^{T}$ in blocks (timeslots) of $N$ symbols. The block's duration is relatively small compared to the involved channels' coherence time. Let us also assume that the SU knows which timeslot is used for a regular PU communication (i.e., when the PU transmitter forwards data to PU receiver) and which one for a reverse PU communication (i.e., when the PU receiver transmits back to the PU transmitter). This is in fact the only knowledge that the SU requires so as to compute the pre/post-coding matrices.

\subsubsection{Estimation of Matrices $\mathrm{Q}_{r}$ and $\mathrm{Q}_{t}$}

First, it is shown that matrix $\mathbf{Q}_{r}$ can be blindly estimated at regular PU timeslots. Let us assume that the PU transmitter employs $L_{p}$ singular channels to transmit its data as described in Subsection 2.3.2. If we consider that the SU transmitter remains silent during this timeslot, then the received signal at the SU receiver is given by

$$
\mathbf{y}_{s r}=\mathbf{H}_{s p} \mathbf{V}_{p}\left(1: T_{p}, 1: L_{p}\right) \mathbf{P}_{p} \mathbf{x}_{p}+\mathbf{z}_{s r}
$$

where $\mathbf{z}_{s r}$ is an $R_{s} \times 1$ noise vector. Under the assumption that $\mathbb{E}\left\{\mathbf{x}_{p} \mathbf{x}_{p}^{H}\right\}=\mathbf{I}_{L_{p}}$, and that $\mathbf{z}_{s r}$ is statistically independent to $\mathbf{x}_{p}$, the covariance matrix of the received data is given by

$$
\mathbf{R}_{s r}=\mathbb{E}\left\{\mathbf{y}_{s r} \mathbf{y}_{s r}^{H}\right\}=\left(\mathbf{H}_{s p} \mathbf{V}_{p}\left(1: T_{p}, 1: L_{p}\right) \mathbf{P}_{p}\right)^{2}+\sigma_{n}^{2} \mathbf{I}_{R_{s}} .
$$

By defining now the $R_{s} \times L_{p}$ matrix $\mathbf{D}_{s p}$ as $\mathbf{D}_{s p}=\mathbf{H}_{s p} \mathbf{V}_{p}\left(1: T_{p}, 1: L_{p}\right) \sqrt{\mathbf{P}_{p}}$ and using its SVD $\mathbf{D}_{s p}=\mathbf{U}_{D_{s p}} \boldsymbol{\Sigma}_{D_{s p}} \mathbf{V}_{D_{s p}}^{H}$ in (2.14) we get

$$
\mathbf{R}_{s r}=\mathbf{U}_{D_{s p}} \Sigma_{D_{s p}}^{2} \mathbf{U}_{D_{s p}}^{H}+\sigma_{n}^{2} \mathbf{I}_{R_{s}}
$$


From equation (2.15) it is evident now that $\mathbf{Q}_{r}$ can be estimated via the noise subspace vectors of the covariance matrix $\mathbf{R}_{s r}$. Thus, the SU receiver computes $\mathbf{Q}_{r}$ by the SVD of the sample covariance matrix, i.e., $\hat{\mathbf{R}}_{s r}=\hat{\mathbf{U}}_{s r} \hat{\Sigma}_{s r} \hat{\mathbf{U}}_{s r}^{H}$, as

$$
\hat{\mathbf{Q}}_{r}=\hat{\mathbf{U}}_{s r}\left(1: R_{s}, L_{p}+1: R_{s}\right)
$$

In a similar manner, matrix $\mathbf{Q}_{t}$ can be blindly estimated at the PU's reverse communication timeslots. Let us assume that the PU receiver employs the $L_{p}$ singular channels to transmit the feedback data $\mathbf{x}_{p}$. If we consider that the $\mathrm{SU}$ receiver remains silent, the received signal at the SU transmitter is given by,

$$
\mathbf{y}_{s t}=\mathbf{H}_{p s}^{T} \mathbf{U}_{p}^{*}\left(1: R_{p}, 1: L_{p}\right) \mathbf{P}_{p} \mathbf{x}_{p}+\mathbf{z}_{s t}
$$

where $\mathbf{z}_{s t}$ is a $T_{s} \times 1$ noise vector and $\mathbf{A}^{T}$ and $\mathbf{A}^{*}$ denote the transpose and the conjugate of a matrix $\mathbf{A}$, respectively. Then, the received data covariance matrix is written as

$$
\mathbf{R}_{s t}=\mathbb{E}\left\{\mathbf{y}_{s t} \mathbf{y}_{s t}^{H}\right\}=\mathbf{V}_{D_{p s}}^{*} \Sigma_{D_{p s}}^{2} \mathbf{V}_{D_{p s}}^{T}+\sigma_{n}^{2} \mathbf{I}_{R_{s}}
$$

where

$$
\mathbf{D}_{p s}=\mathbf{U}_{p}^{H}\left(1: R_{p}, 1: L_{p}\right) \mathbf{H}_{p s} \mathbf{P}_{p}=\mathbf{U}_{D_{p s}} \boldsymbol{\Sigma}_{D_{p s}} \mathbf{V}_{D_{p s}}^{H}
$$

Thus, similarly to the estimation of $\mathbf{Q}_{r}$, the SU transmitter may employ the SVD of the sample covariance matrix, i.e., $\hat{\mathbf{R}}_{s t}=\hat{\mathbf{V}}_{s t} \hat{\boldsymbol{\Sigma}}_{s t} \hat{\mathbf{V}}_{s t}^{H}$, to compute $\mathbf{Q}_{t}$ as

$$
\hat{\mathbf{Q}}_{t}=\hat{\mathbf{V}}_{s t}^{*}\left(1: T_{s}, L_{p}+1: T_{s}\right) .
$$

\subsubsection{Detection of the PU's Signal Subspace Rank}

In the previous subsection we implicitly assumed that the SU knows the number of the employed PU singular channels $L_{p}$ and thus it can determine the required null-spaces' size. If this is not the case, then a rank detection technique must be employed prior the computation of $\mathbf{Q}_{t}$ and $\mathbf{Q}_{r}$. In general, a common approach 
followed in the relevant literature, is to employ Information Theoretic Criteria (i.e. Minimum Description Length (MDL) [89]) in order to detect the rank of the signal subspace. An alternative approach would be to use a sequence of hypothesis tests on the sample covariance matrix's eigenvalues (or on functions of them), in a NeymanPearson sense [90]-[91]. The latter approach is considered more suitable here due to its adaptable nature. For example, let us assume that the interference channels, given by matrices $\mathbf{H}_{s p}$ and $\mathbf{H}_{p s}$, are in relatively bad condition thus degrading the performance of rank detection schemes. The advantage of using an eigenvalue-based hypothesis test is that the corresponding thresholds can be re-designed assuming a greater probability of false alarm $\left(P_{f}\right)$. An increase in the targeted $P_{f}$ value reduces the probability of rank under-estimation and increases the probability of rank-overestimation. Therefore, by designing a less "aggressive" SU system, the PU system transmissions are protected from interference that could be generated by a possible rank under-estimation. Moreover, there exist some useful analytical results concerning the probability of rank-over-estimation for the eigenvalue-based approach which enable us to derive a number of corresponding theoretical results concerning the performance of the SU system. In the present work we properly apply the approach of [90] due to its simplicity. A brief description of the employed rank detection scheme is provided in the following.

Let us assume that the PU (transmitter or receiver) transmits a signal of rank $L_{p}$ and that the SU remains silent. If the ordered eigenvalues of the SU transmitter's (or receiver's) received data covariance matrix are given by $\lambda_{1}, \ldots, \lambda_{T_{s}}$, we define as $\mathcal{H}_{L_{p}}$ the hypothesis that the PU signal's rank is $L_{p}$ i.e.,

$$
\mathcal{H}_{L_{p}}=\lambda_{1} \geq \cdots \geq \lambda_{L_{p}} \geq \lambda_{L_{p}+1}=\cdots=\lambda_{T_{s}}=\sigma_{n}^{2}
$$

Let us now assume that the SU transmitter's (or receiver's) received data covariance matrix is estimated by the corresponding sample covariance matrix by a number of $N$ samples. The authors in [90] have proved that the sum of the noise eigenvalues $S_{L_{p}}$ of the sample covariance matrix, normalized by the noise variance, is approximately 
distributed as a chi-squared variable with $\left(T_{s}-L_{p}\right)\left(N-L_{p}\right)$ degrees of freedom. That is,

$$
\frac{2}{\sigma_{n}^{2}} S_{L_{p}}=\sum_{n=L_{p}+1}^{T_{s}} \lambda_{n} \sim \chi_{\left(T_{s}-L_{p}\right)\left(N-L_{p}\right)}^{2}
$$

Now, if we define $\left\{S_{k}\right\}_{k=0}^{T_{s}-1}$ as the cumulative sums of the last $T_{s}-k-1$ singular values of the sample covariance matrix, we can determine the signal rank by applying the following nested sequence of hypothesis testings

$$
\begin{array}{r}
k^{\text {th }} \text { test }: \mathcal{H}_{k}^{0}: \quad \text { Choose } \mathcal{H}_{0} \cup \cdots \cup \mathcal{H}_{T_{s}-k} \\
\text { if } S_{m-k}<T_{m-k} \\
\mathcal{H}_{k}^{1}: \quad \text { Choose } \mathcal{H}_{T_{s}-m+1} \\
\text { if } S_{m-k} \geq T_{m-k},
\end{array}
$$

Therefore, we start from the $S_{T_{s}-1}$ and keep testing until the $\mathcal{H}_{k}^{1}$ is chosen for some $k$ and then we decide that the rank is $T_{s}-k+1$. The sequence of thresholds $\left\{T_{k}\right\}_{k=0}^{m-1}$ is selected according to a level $\alpha$ such that

$$
\begin{aligned}
P\left(S_{0} \geq T_{0} \mid \mathcal{H}_{0}\right) & =P\left(S_{1} \geq T_{1} \mid \mathcal{H}_{1}\right) \\
& =\cdots=P\left(S_{m-1} \geq T_{m-1} \mid \mathcal{H}_{m-1}\right) \\
& =\alpha .
\end{aligned}
$$

At any given test, $\alpha$ is the corresponding individual false alarm level for that test since $P\left(S_{k} \geq T_{k} \mid \mathcal{H}_{k}\right)$ is the probability of choosing $\mathcal{H}_{k+1}$ when $\mathcal{H}_{k}$ is true. Under hypothesis $\mathcal{H}_{k}$, which corresponds to the case where the rank of the signal subspace of the covariance matrix is $k, S_{k}$ is the energy of the perturbed noise subspace. The thresholds in (2.22) are computed for a predefined probability of false alarm level by using eqs.(2.21)-(2.23). 


\subsubsection{Estimation of Matrices $\mathrm{V}_{z}$ and $\mathrm{U}_{z}$}

The SU system, having estimated matrices $\mathbf{Q}_{t}$ and $\mathbf{Q}_{r}$ can now compute matrices $\mathbf{V}_{z}$ and $\mathbf{U}_{z}$. Let us first see the case of matrix $\mathbf{V}_{z}$. At a reverse $\mathrm{PU}$ communication timeslot, the $\mathrm{SU}$ receiver transmits a data vector pre-coded by $\hat{\mathbf{Q}}_{r}^{*}$ and the $\mathrm{SU}$ transmitter receives a signal which given by

$$
\mathbf{y}_{s t}=\frac{1}{\sqrt{R_{s}-L_{p}}} \mathbf{H}_{s s}^{T} \hat{\mathbf{Q}}_{r}^{*} \mathbf{x}_{s}+\mathbf{H}_{p s}^{T} \mathbf{U}_{p}^{*} \mathbf{P}_{p} \mathbf{x}_{p}+\mathbf{z}_{s t} .
$$

Since $\mathbf{Q}_{r}^{H} \mathbf{H}_{s p} \mathbf{V}_{p}=\mathbf{V}_{p}^{T} \mathbf{H}_{s p}^{T} \mathbf{Q}_{r}^{*}=\mathbf{0}_{T_{p}}$, we can assume that the signal received by the $\mathrm{PU}$ transmitter is interference-free. On the other hand, the signal received by the SU transmitter (see (2.24)) is post-coded by matrix $\hat{\mathbf{Q}}_{t}^{T}$ to remove the interference from the PU receiver since $\mathbf{U}_{p}^{H} \mathbf{H}_{p s} \mathbf{Q}_{t}=\mathbf{Q}_{t}^{T} \mathbf{H}_{p s}^{T} \mathbf{U}_{p}^{*}=\mathbf{0}_{T_{s}}$. That is,

$$
\begin{aligned}
\tilde{\mathbf{y}}_{s t} & =\hat{\mathbf{Q}}_{t}^{T} \mathbf{y}_{s t}=\frac{1}{\sqrt{R_{s}-L_{p}}} \hat{\mathbf{Q}}_{t}^{T} \mathbf{H}_{s s}^{T} \hat{\mathbf{Q}}_{r}^{*} \mathbf{x}_{s}+\tilde{\mathbf{z}}_{s t} \\
& =\frac{1}{\sqrt{R_{s}-L_{p}}} \mathbf{Z}^{T} \mathbf{x}_{s}+\tilde{\mathbf{z}}_{s t} .
\end{aligned}
$$

Now, the corresponding covariance matrix can be written as

$$
\begin{aligned}
\mathbf{R}_{s t} & =\mathbb{E}\left\{\tilde{\mathbf{y}}_{s t} \tilde{\mathbf{y}}_{s t}^{H}\right\}=\frac{1}{R_{s}-L_{p}} \mathbf{Z}^{T} \mathbf{Z}^{*}+\sigma_{n}^{2} \mathbf{I}_{T_{s}} \\
& =\frac{1}{R_{s}-L_{p}} \mathbf{V}_{z}^{*} \Sigma_{z}^{2} \mathbf{V}_{z}^{T}+\sigma_{n}^{2} \mathbf{I}_{T_{s}},
\end{aligned}
$$

where we used the SVD of matrix $\mathbf{Z}=\mathbf{U}_{z} \boldsymbol{\Sigma}_{z} \mathbf{V}_{z}^{H}$. Thus, from eq.(2.26), the sample covariance matrix $\hat{\mathbf{R}}_{s t}=\hat{\mathbf{V}}_{s t} \hat{\boldsymbol{\Sigma}}_{s t} \hat{\mathbf{V}}_{s t}^{H}$ can be used to estimate matrix $\mathbf{V}_{z}$ as $\hat{\mathbf{V}}_{z}=\hat{\mathbf{V}}_{s t}^{*}$. Note that matrix $\hat{\boldsymbol{\Sigma}}_{s t}$ provides an estimation of the singular values of $\mathbf{Z}$ that can be used by the transmitter for the WF-based power allocation scheme.

Finally, in a similar way, it can be shown that matrix $\mathbf{U}_{z}$ can be estimated based on the signal received by the SU receiver during a regular PU communication timeslot. Considering that the precoded symbols $\hat{\mathbf{Q}}_{t} \hat{\mathbf{V}}_{z} \mathbf{P}_{s} \mathbf{x}_{s}$ are transmitted by the $\mathrm{SU}$ 
transmitter, the received signal at the $\mathrm{SU}$ receiver is given by

$$
\mathbf{y}_{s r}=\mathbf{H}_{s s} \hat{\mathbf{Q}}_{t} \hat{\mathbf{V}}_{z} \mathbf{P}_{s} \mathbf{x}_{s}+\mathbf{H}_{s p} \mathbf{V}_{p} \mathbf{P}_{p} \mathbf{x}_{p}+\mathbf{z}_{s r} .
$$

The received signal of eq.(2.27) is post-coded by $\hat{\mathbf{Q}}_{r}^{H}$ so as to remove the interference. Thus, equation (2.27) becomes

$$
\tilde{\mathbf{y}}_{s r}=\hat{\mathbf{Q}}_{r}^{H} \mathbf{y}_{s r}=\mathbf{Z} \hat{\mathbf{V}}_{z} \mathbf{P}_{s} \mathbf{x}_{s}+\tilde{\mathbf{z}}_{s r}
$$

Now the corresponding covariance matrix can be written as

$$
\mathbf{R}_{s r}=\mathbb{E}\left\{\tilde{\mathbf{y}}_{s r} \tilde{\mathbf{y}}_{s r}^{H}\right\}=\mathbf{Z} \mathbf{Z}^{H}+\sigma_{n}^{2} \mathbf{I}_{R_{s}}=\mathbf{U}_{z} \Sigma_{z}^{2} \mathbf{U}_{z}^{H}+\sigma_{n}^{2} \mathbf{I}_{R_{s}}
$$

Therefore, from eq.(2.26), it can be seen that the sample covariance matrix $\hat{\mathbf{R}}_{s r}=$ $\hat{\mathbf{U}}_{s r} \hat{\mathbf{\Sigma}}_{s r} \hat{\mathbf{U}}_{s r}^{H}$ can be used to estimate the required matrix $\mathbf{U}_{z}$ as $\hat{\mathbf{U}}_{z}=\hat{\mathbf{U}}_{s r}$.

Remark 2.6 As it was stated in the beginning of the present section, the $S U$ requires at least knowledge of the type of the PU timeslot (regular or reverse) in order to estimate the required pre/post-coding matrices. Moreover, from time to time the latter matrices may be needed to be re-estimated, for example, in cases where the involved channels undergo a sudden variation. More generally, for several reasons related to the PU network functionalities (periods for synchronization, scheduling etc.) the $S U$ should seize its transmission for a specific amount of time. In general, such problems are addressed by the MAC layer of the network, i.e. with low rate control channels via which the $S U$ is aware of the PU transmissions. MAC protocols, that handle problems similar to the ones raised here, are subjects of current research and several approaches appeared in literature over the last few years [92].

Remark 2.7 Given the fact that all the involved pre/post-coding matrices are computed via the subspace bases of the associated correlation matrices, one may directly extend the proposed approach so as to track the variations of the pre/post-coding matrices by employing well-studied subspace tracking techniques [93]. 


\section{Table 2-I: The Complete Estimation Scheme}

1: (TS 1) PU Regular Communication Timeslot: The SU transmitter remains silent for $N$ symbol slots during which matrix $\mathbf{Q}_{r}$ is estimated

2: $\quad$ Compute $\hat{\mathbf{R}}_{s r}=(1 / N) \sum_{n=1}^{N} \mathbf{y}_{s r}(n) \mathbf{y}_{s r}^{H}(n)$ at the SU receiver.

3: $\quad$ Estimate the Rank $\hat{L}_{p}$ of the PU signal subspace.

$4: \quad$ Set $\hat{\mathbf{Q}}_{r}=\hat{\mathbf{U}}_{s r}\left(1: R_{s}, \hat{L}_{p}+1: R_{s}\right)$ from $\hat{\mathbf{R}}_{s r}=\hat{\mathbf{U}}_{s r} \hat{\boldsymbol{\Sigma}}_{s r}^{2} \hat{\mathbf{U}}_{s r}^{H}$

5: (TS 2) PU Reverse Communication Timeslot:

6: 1st Phase: The SU receiver remains silent for $N_{1}$ symbol slots during which $\mathbf{Q}_{t}$ is estimated

7: $\bullet$ Compute $\hat{\mathbf{R}}_{s t}=(1 / N) \sum_{n=1}^{N} \mathbf{y}_{s t}(n) \mathbf{y}_{s t}^{H}(n)$ at the SU transmitter.

8: $\quad$ - Re-Estimate the Rank $\hat{L}_{p}^{\prime}$ of the PU signal subspace. If $\hat{L}_{p}^{\prime}>\hat{L}_{p}$

inform the SU receiver to set $\hat{\mathbf{Q}}_{r}=\hat{\mathbf{U}}_{s r}\left(1: R_{s}, \hat{L}_{p}^{\prime}+1: R_{s}\right)$

9: $\quad$ Set $\hat{L}_{p}=\max \left\{\hat{L}_{p}, \hat{L}_{p}^{\prime}\right\}$ and $\hat{\mathbf{Q}}_{t}=\hat{\mathbf{V}}_{s t}^{*}\left(1: T_{s}, \hat{L}_{p}+1: T_{s}\right)$ from $\hat{\mathbf{R}}_{s t}=\hat{\mathbf{V}}_{s t} \hat{\Sigma}_{s t}^{2} \hat{\mathbf{V}}_{s t}^{H}$

10: 2nd Phase: The $S U$ receiver transmits data for $N_{2}=N-N_{1}$ symbol slots during which $\mathbf{Q}_{t}$ is estimated

11: $\quad$ The SU receiver transmits an appropriately precoded signal $\hat{\mathbf{Q}}_{r}^{*} \mathbf{x}_{s} / \sqrt{R_{s}-L_{p}}$

12: $\quad$ The SU transmitter post-codes the received signal with matrix $\hat{\mathbf{Q}}_{t}^{T}$

13: - Re-compute the sample covariance matrix at the SU transmitter $\hat{\mathbf{R}}_{s t}$

14: $\bullet$ Set $\hat{\mathbf{V}}_{z}=\hat{\mathbf{V}}_{s t}^{*}$ and $\hat{\Sigma}_{z}=\hat{\Sigma}_{s t}$ from $\hat{\mathbf{R}}_{s t}=\hat{\mathbf{V}}_{s t} \hat{\Sigma}_{s t}^{2} \hat{\mathbf{V}}_{s t}^{H}$

15: (TS 3) PU Regular Communication Timeslot: The transmission of the $S U$ transmitter's data starts, matrix $\mathbf{U}_{z}$ is also estimated

16: - The SU TX transmits an appropriately precoded signal $\hat{\mathbf{Q}}_{t} \hat{\mathbf{V}}_{z} \mathbf{P}_{s} \mathbf{x}_{s}$

17: $\quad$ The $\mathrm{SU}$ receiver post-codes the received signal with matrix $\hat{\mathbf{Q}}_{r}^{H}$

18: - Re-compute the sample covariance matrix at the SU receiver $\hat{\mathbf{R}}_{s r}$

19: $\bullet$ Set $\hat{\mathbf{U}}_{z}=\hat{\mathbf{U}}_{s r}$ from $\hat{\mathbf{R}}_{s r}=\hat{\mathbf{U}}_{s r} \hat{\boldsymbol{\Sigma}}_{s r}^{2} \hat{\mathbf{U}}_{s r}^{H}$ 


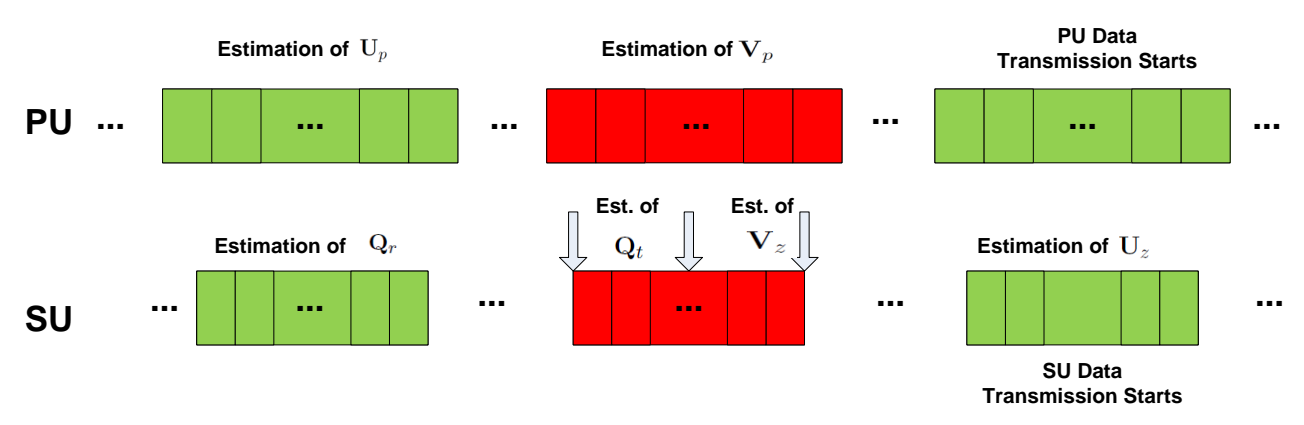

Figure 2-2: Frame Structure Diagram

\subsubsection{The Complete Estimation Scheme}

In Table 2-I the complete estimation procedure is presented. As already discussed, the SU must first determine the rank of the transmitted PU signal (or equivalently the rank of the available null-spaces at the PU and SU receivers) so as to avoid causing interference to (or experiencing interference from) the PU transmitted signals, by properly aligning the SU signals to the corresponding null-spaces. The required rank is determined via the rank detection scheme described in Subsection 2.4.2. Then matrices $\mathbf{Q}_{r}$ and $\mathbf{Q}_{t}$ can be derived by the null-spaces of the received signals sample covariance matrix at the SU ends, as it has already been described in Subsection 2.4.1. Finally, the estimation of matrices $\mathbf{V}_{z}$ and $\mathbf{U}_{z}$ is done according to the steps given in Subsection 2.4.3. The whole estimation procedure can be executed in a sequence of three PU timeslots (TS) in which $N$ data symbols are transmitted. A schematic frame structure diagram is depicted in Fig.2-2. The green timeslots correspond to regular communication and the red ones to reverse communication. Note that the order that the aforementioned timeslots appear in the estimation scheme of Table 2-I and in Fig. 2-2 is not unique. The SU requires only two regular and one reverse timeslot so as to estimate the required pre/post-coding matrices. It is easy to see that for example, the first two (estimation) timeslots may appear in reverse order. Moreover, these three timeslots do not have to be consecutive, provided that the channel variations permit to do so. It should also be stressed that the SU timeslots do not need to be perfectly synchronized since, in the proposed technique, the pre/post-coding matrices may be estimated blindly (hence no synchronization to the PU training slots is actually 
required).

The first one of the three time slots (corresponding to lines 1-4 of Table 2-I), is a PU regular transmission timeslot. The PU transmitter transmits its data to the PU receiver. As it is shown, in this timeslot the $\mathrm{PU}$ can also estimate its post-coding matrix $\mathbf{U}_{p}$ via a pilot-based or a blind method (as it is marked in Figure 2). In any case, since a blind method is employed at the SU side, the SU can always estimate its post-coding matrices independently of what data the PU system transmits. At this timeslot, both the SU transmitter and receiver remain silent. The SU receiver computes the sample covariance matrix $\hat{\mathbf{R}}_{s r}$ and estimates the rank $\hat{L}_{p}$ of the PU signal by using the eigenvalue based rank detection scheme of [90]. Then, it estimates matrix $\mathbf{Q}_{r}$ as described in eqs.(2.13)-(2.16). The second timeslot is a reverse $\mathrm{PU}$ transmission slot (lines 5-14 of Table 2-I) where the PU receiver sends back data to the PU transmitter and it is divided into two phases. In the 1st phase (lines 6-9), of duration $N_{1}$ symbol slots, the SU receiver remains silent and the SU transmitter computes the sample covariance matrix $\hat{\mathbf{R}}_{s r}$ and re-estimates the rank $\hat{L}_{p}^{\prime}$ of the PU signal. In this phase, we assume that there is some type of interaction between the SU receiver and the SU transmitter via a low rate feedback channel in order to determine the rank of the PU signal. That is, the PU signal rank is assumed to be equal to $\hat{L}_{p}=\max \left\{\hat{L}_{p}, \hat{L}_{p}^{\prime}\right\}$ so as to protect the PU transmission from a rank-underestimation event. Then, the SU transmitter, estimates matrix $\mathbf{Q}_{t}$ as described in eqs.(2.17)(2.19). In the 2nd phase (lines 10-14), of duration $N_{2}=N-N_{1}$ symbol slots, the SU receiver transmits the precoded symbols $\left(\hat{\mathbf{Q}}_{r}^{*} \mathbf{x}_{s}\right) / \sqrt{R_{s}-\hat{L}_{p}}$ and the matrices $\mathbf{V}_{z}$ and $\boldsymbol{\Sigma}_{z}$ are estimated at the SU transmitter as described in eqs.(2.24)-(2.26). Note that during this timeslot the PU transmitter can also estimate the pre-coding matrix $\mathbf{V}_{p}$ as it is depicted again in Fig. 2-2.

Finally, the 3rd time slot (lines 15-19) is a PU regular transmission time slot, where the SU transmitter transmits $\hat{\mathbf{Q}}_{t} \hat{\mathbf{V}}_{z} \mathbf{P}_{s} \mathbf{x}_{s}$ and the SU receiver estimates the matrix $\mathbf{U}_{z}$ according to eqs.(2.27)-(2.29). Note that since the $\mathrm{SU}$ transmitter has already estimated $\mathbf{Q}_{t}, \mathbf{V}_{z}$ and $\Sigma_{z}$, the transmission of the $\mathrm{SU}$ data can start at this time slot (i.e., the third one), even though the transmission is used for the estimation 
of $\mathbf{U}_{z}$ at the receiver.

Remark 2.8 From the description of the proposed estimation scheme, it is evident that the $S U$ is now able to compute all required CSI related to the cross SU-PU channels (i.e., the rank of the PU transmitted signals, as well as the matrices $\mathbf{Q}_{r}$ and $\mathbf{Q}_{t}$ ) in a completely blind manner.

Remark 2.9 Matrices $\mathbf{V}_{p}$ and $\mathbf{U}_{p}$ of the PU can also be estimated blindly in a way similar to the one of $\mathbf{V}_{z}$ and $\mathbf{U}_{z}$ as it is already mentioned above. Of course both of the SU and the PU may also apply a training-based or a semi-blind estimation method for the aforementioned matrices in order to improve their performance.

Remark 2.10 As it is known, blind techniques provide estimates with some phase ambiguity. Phase ambiguity does not constitute a problem in the case of the interference cancellation matrices $\mathbf{Q}_{r}$ and $\mathbf{Q}_{t}$, since the multiplication of the latter matrices with a scalar complex number does not affect the null-space they span. However, this is not the case with matrices $\mathbf{V}_{z}$ and $\mathbf{U}_{z}$ where phase ambiguity is indeed a problem unless differential modulation schemes are employed by the SU. If other modulation schemes are used, then it can be shown, that the involved ambiguity can be easily resolved by employing a few training symbols per block of transmission.

\subsection{Performance Analysis}

In this section, a number of results concerning the performance of the proposed OIA technique are presented. First, close approximations of the PU and SU systems' ergodic capacities are provided assuming perfect CSI. Note that in [81] only an asymptotic performance analysis was conducted. Then, we present a theoretical result concerning the interference generated to the PU transmissions due to the imperfect estimation of matrix $\mathbf{Q}_{t}$ at the SU transmitter. Based on the previous results we derive the corresponding theoretical ergodic capacity of the PU system for the imperfect CSI case. Next, a lower bound on the capacity of the SU system is derived when the proposed estimation scheme of Subsection 2.4.4 is employed. Finally, theoretical 
results are presented that provide some insight on the impact of the rank detection schemes to the performance of the SU and PU systems.

\subsubsection{Perfect CSI Case}

First, the ergodic capacity of an arbitrary $T_{x} \times R_{x}$ MIMO system employing a SpaceTime WF algorithm is given by next Theorem.

Theorem 2.1 The ergodic capacity of a $R_{x} \times T_{x}$ MIMO system, employing the SpaceTime WF algorithm, under Rayleigh fading conditions and perfect CSI is given by

$$
\bar{C}\left(\min \left\{T_{x}, R_{x}\right\}, \bar{\beta}, \sigma_{n}^{2}, \overline{\boldsymbol{\lambda}}\right)=\sum_{l=1}^{\min \left\{T_{x}, R_{x}\right\}} \frac{1}{\left(K_{l}-1\right) !} G_{1,3}^{3,2}\left(\begin{array}{c}
1,1 \\
0,0, K_{l}
\end{array} \mid, \frac{K_{l} \sigma_{n}^{2}}{\bar{\beta} \bar{\lambda}_{l}}\right)
$$

where $\overline{\boldsymbol{\lambda}}=\left[\bar{\lambda}_{1}, \ldots, \bar{\lambda}_{L}\right]$ is a vector containing the expected values of the squared singular values, $\sigma_{n}^{2}$ is the noise variance, $\bar{\beta}$ is the water-level threshold computed via the space-time WF algorithm by numerically solving $(2.10), K_{l}=\left(T_{x}-l+1\right)\left(R_{x}-l+1\right)$ and $G_{p, q}^{m, n}$ is the so-called MeijerG function [87].

Proof The proof is given in Appendix 2.A.

Since the pre-coding matrix $\mathbf{Q}_{t}$ nulls-out completely the interference at the PU receiver, the ergodic capacity of the PU system is provided by Theorem 2.1 for $T_{x}=$ $T_{p}$ and $R_{x}=R_{p}$, respectively. In the following we derive the ergodic capacity of the SU system. At first, the concept of the "transmission opportunities number" is introduced.

Definition 2.1 Let us define as transmission opportunities number $T_{\text {opp }}$ the number of unused PU spatial dimensions in which zero power is allocated by the WF algorithm to a specific fading state. That is, for a given noise variance $\sigma_{n}^{2}$ and a WF threshold $\bar{\beta}_{p}$ computed by solving eq.(2.10), $T_{\text {opp }}$ is given by

$$
T_{\text {opp }}=\sum_{l=1}^{\min \left\{T_{p}, R_{p}\right\}} \mathbb{1}_{\left[0, \sigma_{n}^{2} / \bar{\beta}_{p}\right]}\left(\lambda_{l}\right)
$$


where $\lambda_{l}$ is the $l-$ th squared singular value of matrix $\mathbf{H}_{p p}$.

The following Lemma is concerned with the relation between the mean transmission opportunities number $\bar{T}_{o p p}$ and the probability that the WF algorithm allocates zero power to the $l-t h$ PU eigen-channel $\mathcal{P}_{\text {off }}^{(l)}$.

Lemma 2.1 The probability that the WF algorithm allocates zero power to the $l$ - th eigen-channel $\mathcal{P}_{\text {off }}^{(l)}$ is given by

$$
\mathcal{P}_{o f f}^{(l)}=1-\frac{\Gamma\left(K_{l}, \frac{K_{l} \sigma_{n}^{2}}{\bar{\beta}_{p} \bar{\lambda}_{l}}\right)}{\left(K_{l}-1\right) !} .
$$

The mean number of transmission opportunities is given by

$$
\bar{T}_{\text {opp }}=\sum_{l=1}^{\min \left\{T_{p}, R_{p}\right\}} \mathcal{P}_{o f f}^{(l)}
$$

Proof The proof is given in Appendix 2.B.

Finally, based on the results of Theorem 2.1 and Lemma 2.1 the ergodic capacity of the SU system is given by Theorem 2.2.

Theorem 2.2 The ergodic capacity of the SU system is given by

$$
\begin{aligned}
& \bar{C}_{S U}=\sum_{i=1}^{\min \left\{T_{p}, R_{p}\right\}} \prod_{l=1}^{i} \mathcal{P}_{o f f}^{(l)} \prod_{l=i+1}^{\min \left\{T_{p}, R_{p}\right\}}\left(1-\mathcal{P}_{o f f}^{(l)}\right) \times \\
& \bar{C}\left(L_{s}(i), \bar{\beta}_{i}, \sigma_{n}^{2}, \overline{\boldsymbol{\lambda}}_{s}(i)\right),\left(L_{p}, L_{s}, \overline{\boldsymbol{\beta}}_{s}, \overline{\boldsymbol{\Lambda}}_{s}\right)
\end{aligned}
$$

where $\bar{\beta}_{p}$ is the PU water-level threshold, $L_{s}(i)=\min \left\{\left(T_{s}-\min \left\{T_{p}, R_{p}\right\}+i\right),\left(R_{s}-\right.\right.$ $\left.\left.\min \left\{T_{p}, R_{p}\right\}+i\right)\right\}, \bar{\beta}_{i}$ and $\overline{\boldsymbol{\lambda}}_{s}(i)$ is the water-level threshold and the expected values vector of the squared singular values of a $\left(T_{s}-\min \left\{T_{p}, R_{p}\right\}+i\right),\left(R_{s}-\min \left\{T_{p}, R_{p}\right\}+i\right)$ SU system, respectively.

Proof The proof is given in Appendix 2.C. 


\subsubsection{The Imperfect CSI Case with Perfect PU Rank Knowl- edge}

In this subsection we study the performance of the PU and SU systems when the blind scheme of Table 2-I is employed for estimating the required pre- and postcoding matrices at the SU system. For simplicity, the analysis is conducted assuming perfect knowledge of the PU signal rank at the SU. In Subsection 2.5.3 we examine the impact of the rank detection scheme on the performance of the PU and SU systems.

Let us consider now the imperfect CSI case where the blind estimation scheme of Table 2-I provides imperfect estimates of matrices $\mathbf{Q}_{t}, \mathbf{Q}_{r}, \mathbf{V}_{z}, \mathbf{U}_{z}$ and $\boldsymbol{\Sigma}_{z}$ which can be modelled as $\hat{\mathbf{Q}}_{t}=\mathbf{Q}_{t}+\delta \mathbf{Q}_{t}, \hat{\mathbf{Q}}_{r}=\mathbf{Q}_{r}+\delta \mathbf{Q}_{r}, \hat{\mathbf{V}}_{z}=\mathbf{V}_{z}+\delta \mathbf{V}_{z}, \hat{\mathbf{U}}_{z}=\mathbf{U}_{z}+\delta \mathbf{U}_{z}$ and $\hat{\boldsymbol{\Sigma}}_{z}=\boldsymbol{\Sigma}_{z}+\delta \boldsymbol{\Sigma}_{z}$ respectively, where with $\delta \mathbf{X}$ we denote the estimation error of $\mathbf{X}$. At first, the impact of the imperfect estimation of matrix $\mathrm{Q}_{t}$ on the $\mathrm{PU}$ performance is investigated. In the following analysis, it is assumed that the PU has perfect knowledge of its own pre- and post-coding matrices. By applying the perturbation expression $\hat{\mathbf{Q}}_{t}=\mathbf{Q}_{t}+\delta \mathbf{Q}_{t}$ in (2.5) and keeping only the first order terms, we get

$$
\mathbf{y}_{p}=\boldsymbol{\Sigma}_{p} \mathbf{P}_{p} \mathbf{x}_{p}+\mathbf{U}_{p}^{H} \mathbf{H}_{p s} \delta \mathbf{Q}_{t} \mathbf{V}_{z} \mathbf{P}_{s} \mathbf{x}_{s}+\tilde{\mathbf{z}}_{p}
$$

Thus, due to estimation errors, the SU interference is not perfectly nulled-out at the PU since the received signal includes the term $\delta \mathbf{I}_{s}=\mathbf{U}_{p}^{H} \mathbf{H}_{p s} \delta \mathbf{Q}_{t} \mathbf{V}_{z} \mathbf{P}_{s} \mathbf{x}_{s}$. The following Lemma provides an asymptotic result concerning the mean interference power to the PU system due to the imperfect estimation of $\mathbf{Q}_{t}$.

Lemma 2.2 A first order approximation of the mean interference power generated to the PU transmissions due to imperfect estimation of matrix $\mathbf{Q}_{t}$, as $S N R \rightarrow \infty$ and $N \rightarrow \infty$, and assuming that $L_{s}=\min \left\{T_{s}, R_{s}\right\}>\min \left\{T_{p}, R_{p}\right\}=L_{p}$, is given by

$$
\mathbb{E}\left\{\left\|\mathbf{I}_{p}\right\|^{2}\right\} \approx \frac{\bar{P}_{s} L_{p} \sigma_{n}^{2}}{N}
$$

Proof The proof is given in Appendix 2.D.

Remark 2.11 The ergodic capacity of the PU system, under imperfect SU-related CSI, can be evaluated using Theorem 2.1 and considering as noise-plus-interference power the sum $\tilde{\sigma}_{n}^{2}=\sigma_{n}^{2}+\mathbb{E}\left\{\left\|\delta \mathbf{I}_{s}\right\|^{2}\right\}$. 
Next, the effect of estimation errors on the performance of the SU system is investigated by applying the perturbation equations $\hat{\mathbf{Q}}_{t}=\mathbf{Q}_{t}+\delta \mathbf{Q}_{t}, \hat{\mathbf{Q}}_{r}=\mathbf{Q}_{r}+\delta \mathbf{Q}_{r}$, $\hat{\mathbf{V}}_{z}=\mathbf{V}_{z}+\delta \mathbf{V}_{z}$ and $\hat{\mathbf{U}}_{z}=\mathbf{U}_{z}+\delta \mathbf{U}_{z}$ to (2.11). That is, we have

$$
\mathbf{y}_{s}=\boldsymbol{\Sigma}_{z} \mathbf{P}_{s} \mathbf{x}_{s}+\mathbf{F} \mathbf{P}_{s} \mathbf{x}_{s}+\delta \mathbf{Q}_{r}^{H} \mathbf{H}_{s p} \mathbf{V}_{p} \mathbf{P}_{p} \mathbf{x}_{p}+\tilde{\mathbf{z}}_{s}
$$

where

$$
\mathbf{F}=\boldsymbol{\Sigma}_{z} \mathbf{V}_{z} \delta \mathbf{V}_{z}^{H}+\delta \mathbf{U}_{z}^{H} \mathbf{U}_{z} \boldsymbol{\Sigma}_{z}+\delta \mathbf{U}_{z}^{H} \mathbf{U}_{z} \boldsymbol{\Sigma}_{z} \mathbf{V}_{z}^{H} \delta \mathbf{V}_{z}
$$

The first term is the desired parallel channel decomposition of the perfect CSI case. Due to estimation errors, the channels are no longer independent and are corrupted by inter-channel interference given by the second term of eq. (2.38). The third term is the remaining $\mathrm{PU}$ interference due to imperfect estimation of matrix $\mathbf{Q}_{r}$ (similar to the case of matrix $\mathbf{Q}_{t}$ ). Let us now assume for simplicity, that the elements of matrices $\delta \mathbf{U}_{z}$ and $\delta \mathbf{V}_{z}$ are independent and identically distributed random variables with zero mean and a covariance matrix which is diagonal with $\sigma_{\delta}^{2}(l)$ at its $l-t h$ diagonal entry. The latter assumption regarding the estimation errors, corresponds to the worst case scenario and can be used to derive a lower bound on the capacity of the SU system [94]. For simplicity, we assume that the variance of the interference generated by the imperfect estimation of matrix $\mathbf{Q}_{r}$ is given by re-casting the results of Lemma 2.2 to the SU case. Now, observe that the random variables $\mathbf{V}_{z} \delta \mathbf{V}_{z}^{H}$ and $\delta \mathbf{U}_{z}^{H} \mathbf{U}_{z}$ share the same statistical properties with the $\delta \mathbf{V}_{z}$ and $\delta \mathbf{U}_{z}$, respectively, due to the orthonormal structure of matrices $\mathbf{V}_{z}$ and $\mathbf{U}_{z}$. Therefore, from eq.(2.38) it is easy to compute the variance of the interference generated by the imperfect estimation of matrices $\mathbf{Q}_{r}, \mathbf{V}_{z}$ and $\mathbf{U}_{z}$ to the $l$ - th $\mathrm{SU}$ singular channel, by considering that the SU applies equal power allocation strategy. Thus, by ignoring second and higher order terms, the aforementioned interference variance is given by

$$
\sigma_{I}^{2}(l)=\frac{\sigma_{\delta}^{2}(l) \bar{P}_{s}}{L_{s}-L_{p}} \sum_{k=1}^{L_{s}-L_{p}} \xi_{k}+\sigma_{\delta}^{2}(l) \xi_{l} \bar{P}_{s}+\frac{\bar{P}_{p} \sigma_{n}^{2} L_{s}}{N}
$$

where $L_{s}=\min \left\{T_{s}, R_{s}\right\}, L_{p}$ is the number of the employed PU eigen-channels and 
$\xi_{l}$ is the $l-t h$ squared eigenvalue of the SU system equivalent matrix Z . Note that, by using the results of [95] and [96], where second order statistics of the estimation errors of the sample covariance eigenvectors are derived, we can obtain the following approximation for $\sigma_{\delta}^{2}(l)$. That is,

$$
\sigma_{\delta}^{2}(l)=\frac{\xi_{l}}{N} \sum_{\substack{k=1 \\ k \neq l}}^{L_{s}-L_{p}} \frac{\xi_{k}}{\left(\xi_{i}-\xi_{k}\right)^{2}}
$$

Thus from eqs.(2.37)-(2.40), we can deduce that the SU capacity is lower bounded by the capacity of a MIMO system of $L_{s}-L_{p}$ parallel independent channels. The gain of each channel is the $l-t h$ eigenvalue of the equivalent $\mathrm{SU}$ channel matrix $\mathbf{Z}$ and it is assumed to be corrupted by noise of power $\sigma_{n}^{2}$ plus interference of power $\sigma_{I}^{2}(l)$. That is

$$
C_{S U}^{i m p} \geq \sum_{l=1}^{L_{s}-L_{p}} \log _{2}\left(1+\frac{\xi_{l} \bar{P}_{s}}{\left(L_{s}-L_{p}\right)\left(\sigma_{n}^{2}+\sigma_{I}^{2}(l)\right)}\right)
$$

Deriving a corresponding bound for the ergodic capacity of the SU system, under imperfect CSI, remains, an open issue due to the fact that $\sigma_{I}^{2}(l)$ is a complicated function of the eigenvalues of matrix $\mathbf{Z}$, as it can be seen from eqs.(2.39)-(2.40).

\subsubsection{The Imperfect CSI Case with Imperfect Knowledge of PU's Signal Subspace Rank}

In this subsection, the impact of the PU signal subspace rank detection scheme on the performance of the proposed OIA technique is studied. The study will be performed for two separate cases, the rank over-estimation case and the rank under-estimation one.

Let us assume that at a specific fading state the PU employs $L_{p}$ eigen-channels for its transmission. A rank under-estimation event occurs when the estimated PU rank is $\hat{L}_{p}<L_{p}$. That is, the $\mathrm{SU}$ erroneously aligns its transmissions to a subspace that includes some of the PU signals dimensions, and the resulting interference corrupts the corresponding PU eigen-channels. Moreover, the SU transmissions, employing the SU 
channels that belong to the PU signal subspace, are also corrupted by the interference generated by the PU transmissions. A way to decrease the rank under-estimation probability is to compute the thresholds of the nested sequence of hypothesis tests eq.(2.22) for a greater probability of false alarm $\mathcal{P}_{f}$. Fortunately, as it is depicted in simulations section (Section 2.6), for a large number of samples $N$, the probability of rank under-estimation has non-negligible value only at very low SNR regimes even for a rather small value of $\mathcal{P}_{f}$. Moreover, at the low SNR regimes, the capacity of the systems is in general degraded by the high noise power and therefore the impact of the rank-underestimation on the performance of the systems is negligible. The latter statement is also verified by the simulations. Unfortunately, we cannot derive a closed result concerning the performance of the PU and SU systems due to the fact that there is no obvious way to compute theoretically the probability of rank under-estimation, since the latter requires the knowledge of the distribution function of the $l-t h$ eigenvalue of the sample covariance matrix under the hypothesis that the signal subspace has rank $L_{p}$, which, in general, is unknown.

We consider now the case where a rank over-estimation event occurs when the estimated PU rank is $\hat{L}_{p}>L_{p}$. Clearly, the latter event has no impact on the PU performance since the SU aligns its transmissions to a subspace of smaller dimensions than the ones of the available null-space. As we increase the value of the $\mathcal{P}_{f}$ which is used to compute the decision thresholds for the hypothesis tests, the probability of rank over-estimation is also increased and the SU performance is further degraded. In order to gain further insight regarding the impact of the rank over-estimation events on the performance of the SU system, the ergodic capacity of the SU system is derived by assuming that matrices $\mathbf{V}_{z}$ and $\mathbf{U}_{z}$ are perfectly known at the $\mathrm{SU}$ ends, and that matrices $\mathbf{Q}_{t}$ and $\mathbf{Q}_{r}$ are computed via the proposed estimation scheme. According to [91], the probability of rank over-estimation $\mathcal{P}_{\text {ov }}$ is given by $\mathcal{P}_{\text {ov }} \approx \mathcal{P}\left\{\hat{L}_{p}=L_{p}+1\right\}=$ $\mathcal{P}_{f}$. Now, as it is described in Subsection 2.4.4 and Table 2-I, the proposed estimation scheme runs the rank detection tests at both the SU receiver and the SU transmitter and sets the rank of the PU signal as the maximum of the results of the two previous tests, so as to protect the PU signal from a rank under-estimation event. Now, if 
we consider that the samples number $N$ is large enough (so that the probability of rank under-estimation tends rapidly to zero as SNR increases), then without loss of generality we may assume that each one of the SU ends detects correctly the rank of the PU signal with probability which approximately equals $1-\mathcal{P}_{f}$. Given that both the SU ends must correctly detect the rank of the PU signal, the overall probability of correct rank detection can be approximated by $\mathcal{P}_{d} \approx\left(1-\mathcal{P}_{f}\right)\left(1-\mathcal{P}_{f}\right)$. Now, since a rank over-estimation event, at least at one of the SU ends, leads the whole SU transceiver to an over-estimation event, the probability of rank over-estimation can be approximated as the sum of the probabilities that both the SU ends have a rank over-estimation event $\left(\mathcal{P}_{f}^{2}\right)$ plus the probabilities that one out of them has a rank over-estimation event and one has found the correct rank of the signal $\left(\left(1-\mathcal{P}_{f}\right) \mathcal{P}_{f}\right)$. That is, the overall probability of rank over-estimation may be approximately given by $\mathcal{P}_{\text {ov }} \approx \mathcal{P}_{f}^{2}+2\left(1-\mathcal{P}_{f}\right) \mathcal{P}_{f}$.

Therefore, it is clear that we can assume that the SU system transmits its data through an equivalent MIMO system of dimensions $\left(T_{s}-L_{p}-1\right) \times\left(R_{s}-L_{p}-1\right)$ with probability $\mathcal{P}_{o v}$, and through an equivalent MIMO system of dimensions $\left(T_{s}-\right.$ $\left.L_{p}\right) \times\left(R_{s}-L_{p}\right)$ with probability $\mathcal{P}_{d}$. By the previous discussion and the results of Theorem 2.2 and Lemma 2.2 the following theorem has just been proved which provides the ergodic capacity of the SU system when the rank detection scheme is used to determine the rank of the PU signal.

Theorem 2.3 The ergodic capacity of the SU system, when the PU signal rank and matrices $\mathbf{Q}_{t}$ and $\mathbf{Q}_{r}$ are estimated by the estimation scheme of Table 2-I, and matrices $\mathbf{V}_{z}$ and $\mathbf{U}_{z}$ are perfectly known to the SU system, is given by

$$
\begin{aligned}
\bar{C}_{S U}= & \sum_{i=1}^{\min \left\{T_{p}, R_{p}\right\}} \prod_{l=1}^{i} \mathcal{P}_{o f f}^{(l)} \prod_{l=i+1}^{\min \left\{T_{p}, R_{p}\right\}}\left(1-\mathcal{P}_{o f f}^{(l)}\right) \times \\
& {\left[\mathcal{P}_{d} \bar{C}_{i}+\mathcal{P}_{o v} \bar{C}_{i+1}\right] }
\end{aligned}
$$

where $\bar{C}_{i}=\bar{C}\left(L_{s}(i), \bar{\beta}_{i}, \sigma_{n}^{2}+\sigma_{I}^{2}, \overline{\boldsymbol{\lambda}}_{s}(i)\right), \mathcal{P}_{o v} \approx \mathcal{P}_{f}^{2}+2\left(1-\mathcal{P}_{f}\right) \mathcal{P}_{f}, \mathcal{P}_{d} \approx\left(1-\mathcal{P}_{f}\right)(1-$ $\left.\mathcal{P}_{f}\right), \sigma_{I}^{2}=\bar{P}_{p} \sigma_{n}^{2} / N$ from Lemma 2.2 and the rest of the variables are defined as in 
Theorem 2.2.

Remark 2.12 In case that matrices $\mathbf{V}_{z}$ and $\mathbf{U}_{z}$ are also computed by the estimation scheme of Table 2-I, eqs.(2.39)-(2.41) still provide a lower bound on the capacity of the $S U$ system, as long as we replace the actual PU signal rank $L_{p}$ with the estimated one $\hat{L}_{p}$.

\subsection{Simulation Results}

In this section some indicative simulation results are provided. Let us assume that a PU and a SU system coexist in the same frequency band. For example we can consider a set-up in terms of cellular systems where in the same cell there exist a licensed PU base-station and an unlicensed SU base-station that serve each of them the corresponding PUs and SUs respectively. The aim is to study the performance of both systems in terms of the achieved capacity under the proposed technique for the perfect and the imperfect CSI case. Let us assume also Rayleigh fading conditions and $P_{s}=P_{p}=\bar{P}_{s}=\bar{P}_{p}=1$.

In Fig. 2-3, the performance of the proposed technique is compared to the one of [81] in terms of their achieved ergodic capacity for a $3 \times 3$ PU system under perfect CSI and different values of SNR. We consider that a $6 \times 6$ SU system transmits at the same spectrum area having constant $S N R=20 \mathrm{~dB}$. In the same figure we plot also the theoretical curve that corresponds to the results of Theorem 2.1 and the curve that corresponds to the ergodic capacity of a typical $3 \times 3$ MIMO system without an interfering SU in the environment. As it is evident, all the curves practically coincide thus verifying that the existence of the SU system has no impact on the performance of the PU one.

In Figs. 2-4 and 2-5 the SU's performance of the proposed technique is compared to the one of [81] under perfect CSI. We consider two cases, one with a $3 \times 3 \mathrm{PU}$ system and a $3 \times 3 \mathrm{SU}$ system, and another one with a $3 \times 3 \mathrm{PU}$ and a $6 \times 6 \mathrm{SU}$ system. The PU system has a constant SNR. In Fig.4, the PU link has $S N R=0 d B$

whereas in Fig.5 it has $S N R=20 d B$. In general, as $\min \left\{T_{s}, R_{s}\right\}>\min \left\{T_{p}, R_{p}\right\}$, the 


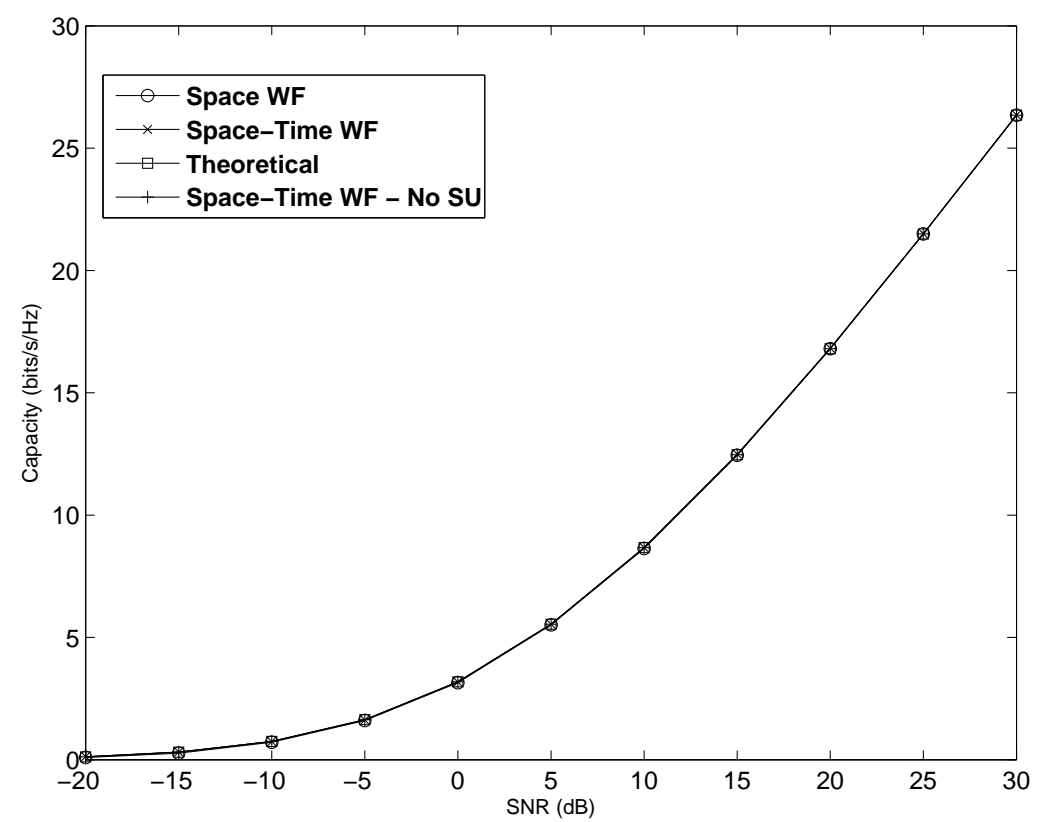

Figure 2-3: Performance of PU system under perfect CSI and SU SNR $=20 \mathrm{~dB}$

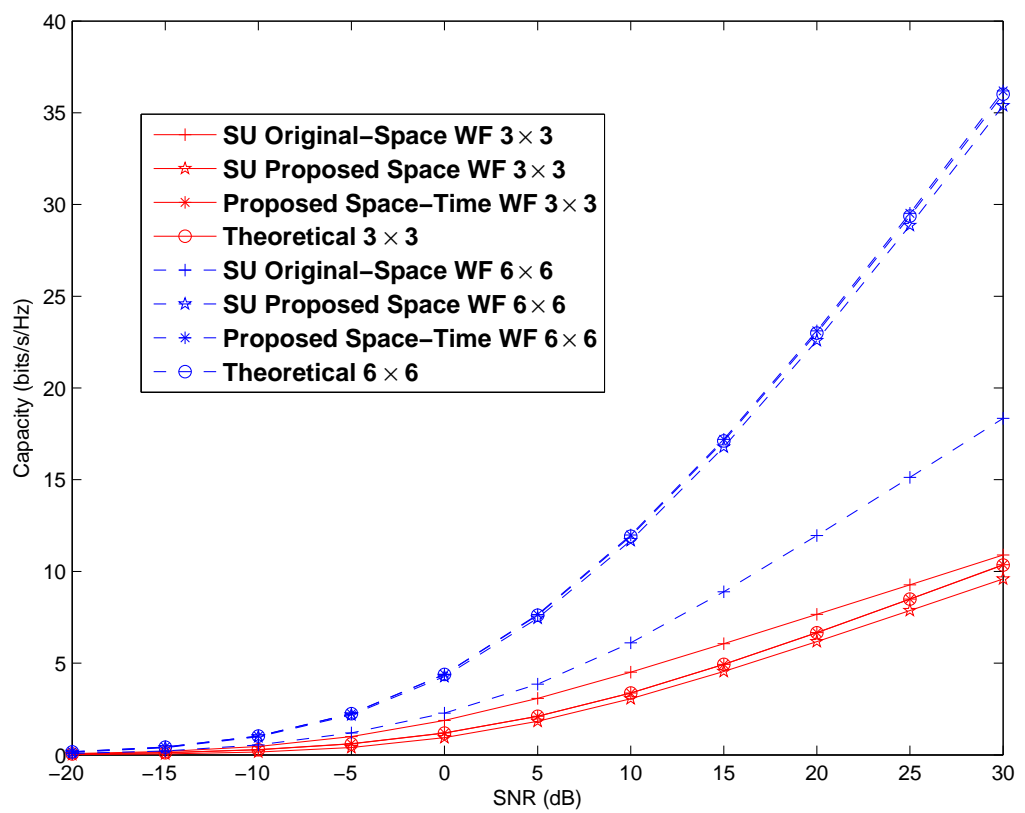

Figure 2-4: Performance of SU system under perfect CSI and PU SNR $=0 \mathrm{~dB}$

proposed technique exhibits a superior performance due to the fact that it nulls-out the PU interference in contrast to the SINR maximization approach of the original technique. On the other hand, the interference nulling approach requires additional degrees of freedom and thus the original technique achieves better performance in cases where $\min \left\{T_{s}, R_{s}\right\} \sim \min \left\{T_{p}, R_{p}\right\}$. 


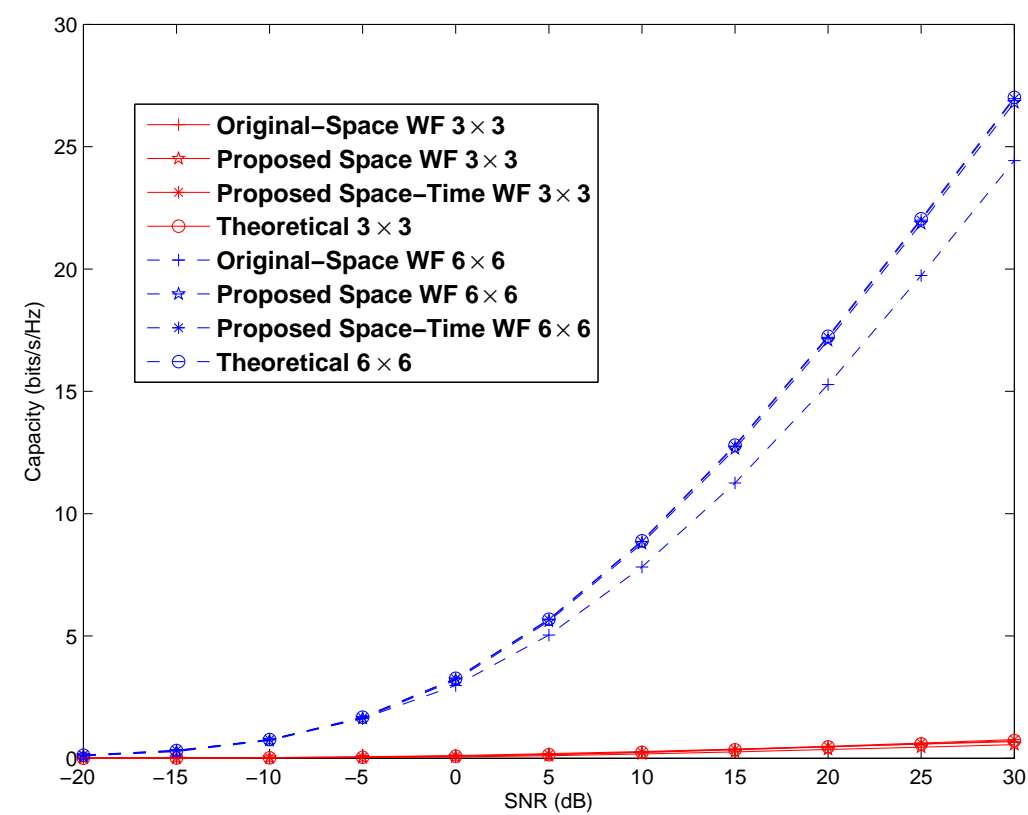

Figure 2-5: Performance of SU system under perfect CSI and PU SNR $=20 \mathrm{~dB}$

Note also that the PU performance of the proposed technique is similar for both cases of space and space-time WF. Moreover, observe that the SU achieves slightly better performance when the space-time WF algorithm is employed. Also, the simulation curves coincide with the theoretical ones given by Theorems 2.1-2.2. Moreover, as it is evident from Fig. 2-4 and 2-5, the gap between the proposed approach and the one of [81], increases with the decrease of the PU SNR. This is explained by the fact that in the proposed approach the extra degrees of freedom that are available due to the bad PU channels' condition are interference free improving more the performance of the SU system.

In order to gain further insight, the transmission opportunities (see Definition 2.1) are presented in Fig. 2-6. As it can be seen, the space-time version provides, in general, more transmission opportunities when the PU channel is at relatively bad condition (i.e. at low-SNR regimes). The latter observation is simply explained by the way the two algorithms work. The space-only version algorithm, allocates always power to the first eigen-channel no matter what is its condition. On the contrary, the space-time version can sometimes allocate zero power to all eigen-channels without degrading the performance of the PU system in terms of the achieved ergodic capacity and thus it may provide an increased number of available degrees of freedom to the 


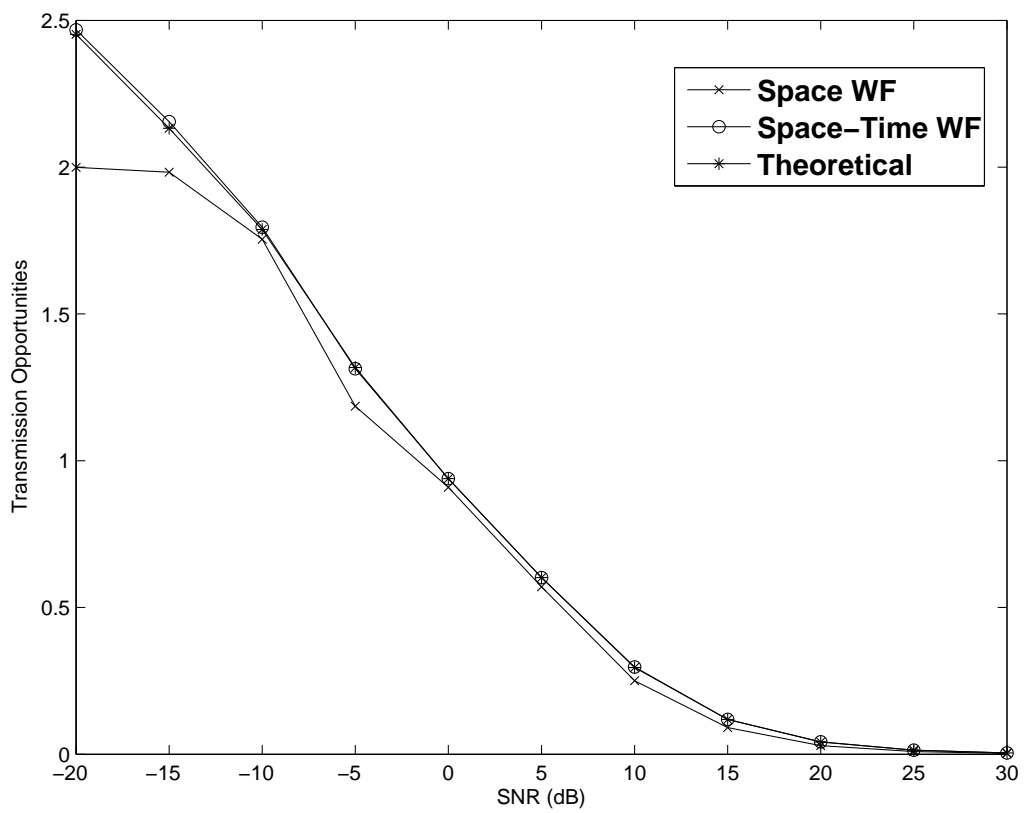

Figure 2-6: Transmission Opportunities

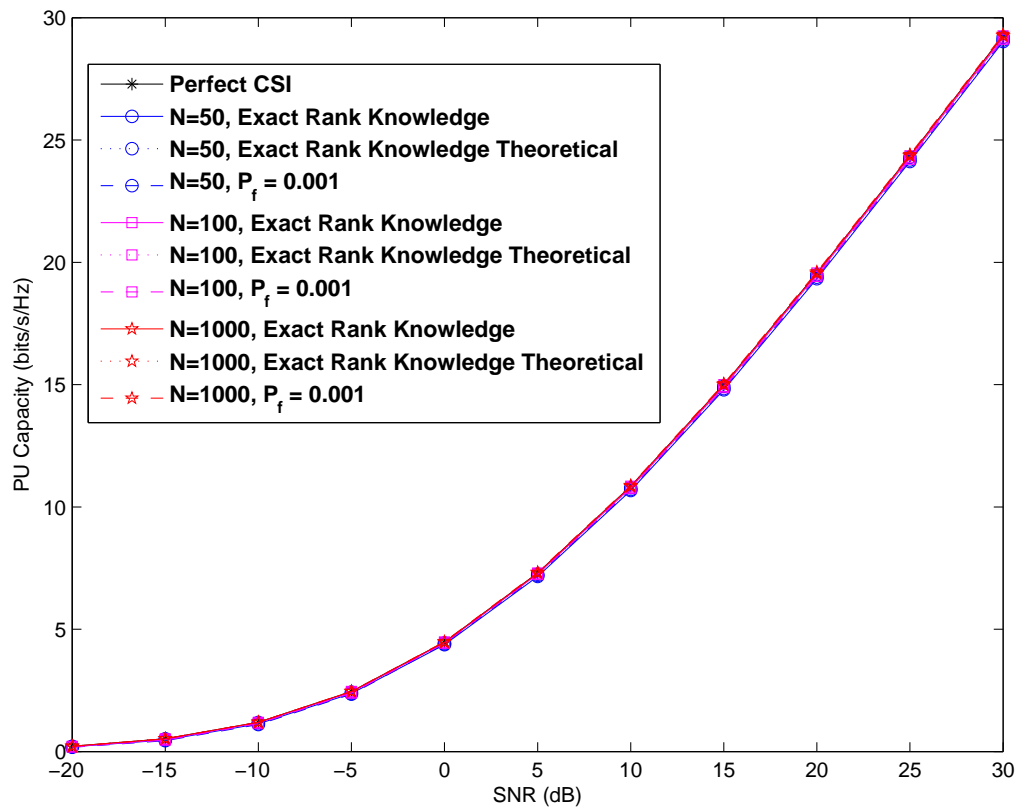

Figure 2-7: Performance of the PU system under imperfect CSI at SU

SU transmission. In the same figure we plot the theoretical results of Lemma 2.1 and it is clear that the theoretical values of transmission opportunities are almost identical to the experimental ones.

In Figs. 2-7 and 2-8 we consider the $3 \times 3 \mathrm{PU}$ and $6 \times 6 \mathrm{SU}$ system under imperfect CSI (blindly estimated). The PU and SU are assumed to transmit BPSK 


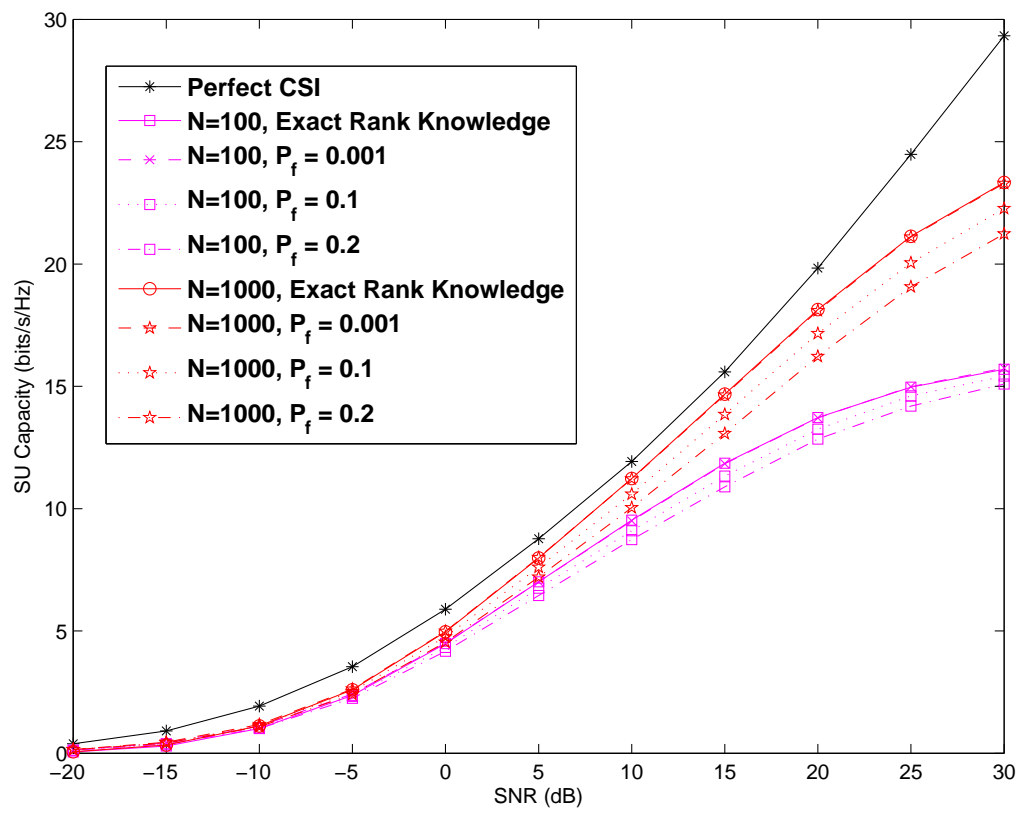

Figure 2-8: Performance of the SU system under imperfect CSI at SU

modulated data symbols in blocks of size $N$, and the corresponding received samples are used to compute the required sample covariance matrices as described in Section 2.4.4. First, we examine the impact of the imperfect estimation of matrix $\mathbf{Q}_{t}$ on the capacity of the PU system. In Fig. 2-7, the PU ergodic capacity is depicted for $N=50,100,1000, P_{f}=0.001$ and $N_{1}=0.3 N$. In the same figure, we present also the PU ergodic capacity when the PU signal subspace rank is known and the corresponding theoretical PU ergodic capacity is computed as described in Remark 2.11. As it can be seen, the theoretical results are very close to the ones obtained via simulations. It is noteworthy that even for a small value of $N$, the blind estimation procedure provides very accurate estimations of matrix $\mathbf{Q}_{t}$.

Next, the impact of the imperfect estimation of matrices $\mathbf{Q}_{r}, \mathbf{V}_{z}$ and $\mathbf{U}_{z}$ on the SU performance is examined. In Fig. 2-8 we present the lower bound for the SU capacity (see eq.(2.41)) under imperfect CSI for $N=100,1000$ and different values of probability of false alarm $\mathcal{P}_{f}=0.01,0.1,0.2$ (Remark 2.12). For comparison purposes we plot the lower bound for the PU ergodic capacity when perfect PU signal subspace rank knowledge is assumed. As it can be seen, the estimation errors of matrices $\mathbf{V}_{z}$ and $\mathbf{U}_{z}$ degrade significantly the $\mathrm{SU}$ performance. It is noteworthy 


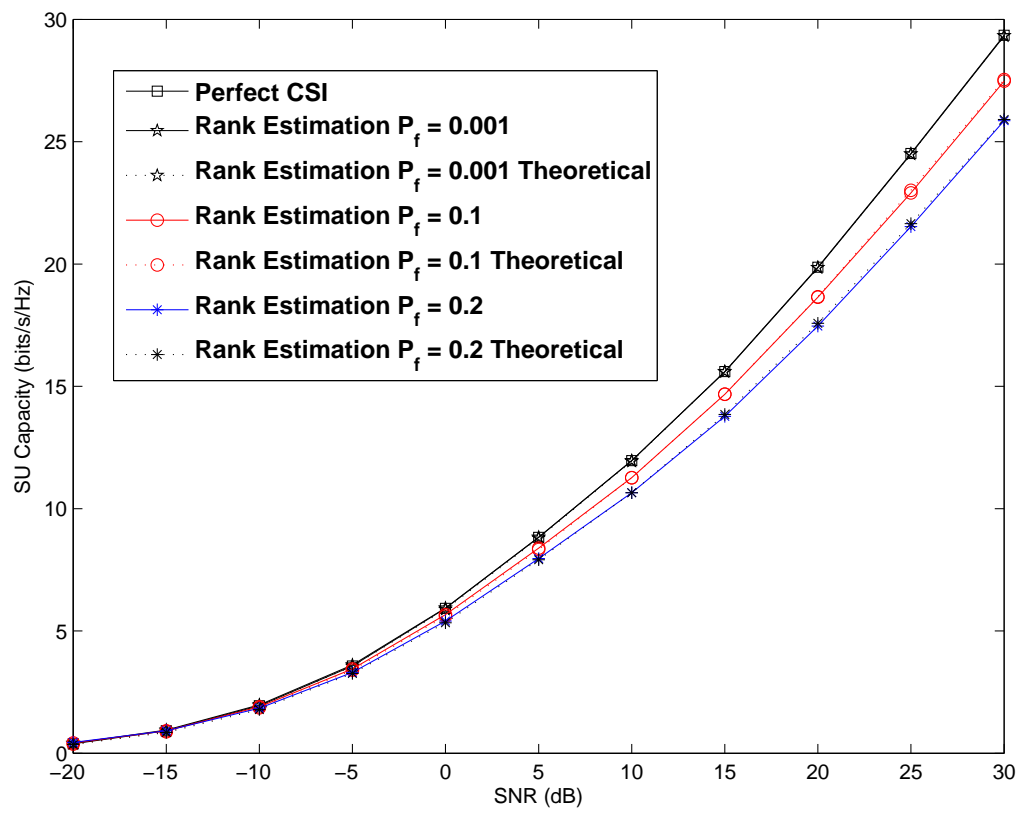

Figure 2-9: Impact of the PU's signal subspace rank detection scheme on the performance of the SU system

that the estimation errors of matrices $\mathbf{Q}_{r}$ and $\mathbf{Q}_{t}$ have smaller impact on the system performance than the ones of matrices $\mathbf{U}_{z}$ and $\mathbf{V}_{z}$. Note that the estimation errors in the latter matrices destroy their diagonal structure which implies that the signals at the receiver are corrupted by inter-channel interference and cannot be perfectly decoupled. Note that the larger the spatial dimension of the system is, the greater is the inter-channel interference, and therefore larger data blocks sizes $N$ are required in order to approximate the perfect CSI performance. Moreover, as we increase the $\mathcal{P}_{f}$ value, we observe that the lowest bound is decreasing due to the simultaneous increase in the probability of rank over-estimation, as stated in Subsection 2.5.3. That is, the remaining interference at the $\mathrm{PU}$ receiver is in general negligible compared to the noise variance and therefore it has almost no impact on the achieved PU capacity. The latter statement is in accordance with Lemma 2.2. (see eqs.(2.53) and (2.54)).

Finally, in order to gain better insight in the performance of the $\mathrm{PU}$ and $\mathrm{SU}$ systems when the PU signal's rank estimation procedure is employed, and verify the results of Theorem 2.3, we consider the same experimental set-up with the one of Figs. 2-7 and 2-8, however, now, we assume that matrices $\mathbf{V}_{z}$ and $\mathbf{U}_{z}$ are perfectly 
known at the SU transmitter and receiver, respectively. In Fig. 2-9, the SU ergodic capacity is depicted for $N=1000$ along with the theoretical results of Theorem 2.3 concerning the SU ergodic capacity. For comparison purposes we present in the same figure the ergodic capacity of the SU system when the rank of the PU signal $L_{p}$ is considered known. As it was described in Subsection 2.5.3, and verified by the results depicted in Fig. 2-7, the PU system does not exhibit any degradation in performance. On the other hand, as $\mathcal{P}_{f}$ increases the capacity of the SU system is degraded, as shown in Figs. 2-8 and 2-9. Note, that the theoretical results for the SU ergodic capacity (Theorem 2.3) are very close to the experimental ones.

\subsection{Conclusion}

In this Chapter a Blind OIA technique for Cognitive Radio systems was derived. First, the OIA technique of [81] was extended by properly re-designing the post-coding matrix of the SU receiver, so as to combat PU interference in a more effective manner. Unlike the original approach, the new one enables the SU to compute blindly the required CSI. To this end, a blind estimation scheme was derived. The performance of the proposed technique under perfect CSI was theoretically studied by computing close approximations of PU and SU ergodic capacities. The performance of the new technique was also studied under imperfect CSI, and lower bounds on the SU capacity were obtained along with some useful theoretical results under different assumptions regarding the known CSI at the SU system. Finally, the performance of the proposed technique was evaluated via extensive simulations in which the corresponding theoretical results were also verified. 


\subsection{Appendices}

\section{A Proof of Theorem 2.1}

The instantaneous capacity of a MIMO system is given in [82] as

$$
C_{l}\left(p_{p}^{(l)}, \sigma_{n}^{2}, \boldsymbol{\lambda}\right)=\sum_{l=1}^{\min \left\{T_{x}, R_{x}\right\}} \log _{2}\left(1+\frac{p_{p}^{(l)} \lambda_{l}}{\sigma_{n}^{2}}\right)
$$

where $p_{p}^{(l)}$ is given by eq.(2.9) and $\boldsymbol{\lambda}=\left[\lambda_{1}, \ldots, \lambda_{\min \left\{T_{x}, R_{x}\right\}}\right]$. From the previous equation and the approximation of [88] for the marginal pdf $f\left(\lambda_{l}\right)$ of the $l-t h$ squared singular value $\lambda_{\mathbf{l}}$, we can derive the ergodic capacity of a MIMO system by

$$
\begin{aligned}
& \bar{C}_{l}\left(\min \left\{T_{x}, R_{x}\right\}, \bar{\beta}, \sigma_{n}^{2}, \overline{\boldsymbol{\lambda}}\right) \\
& =\mathbb{E}_{\boldsymbol{\lambda}}\left\{\sum_{l=1}^{\min \left\{T_{x}, R_{x}\right\}} \log _{2}\left(1+\frac{p_{p}^{(l)} \lambda_{l}}{\sigma_{n}^{2}}\right)\right\} \\
& =\sum_{l=1}^{\min \left\{T_{x}, R_{x}\right\}} \int_{0}^{\infty} \log _{2}\left(1+\frac{p_{p}^{(l)} \lambda_{l}}{\sigma_{n}^{2}}\right) f\left(\lambda_{l}\right) d \lambda_{l} \\
& =\sum_{l=1}^{\min \left\{T_{x}, R_{x}\right\}} \int_{\frac{\sigma_{n}^{2}}{\beta}}^{\infty} \log _{2}\left(\frac{\bar{\beta} \lambda_{l}}{\sigma_{n}^{2}}\right) \frac{1}{\left(K_{l}-1\right) !} \frac{\lambda_{l}^{K_{l}-1}}{\bar{\lambda}_{l}^{K_{l}}} \exp \left(-\frac{\lambda_{l}}{\bar{\lambda}_{l}}\right) d \lambda_{l} \\
& =\sum_{l=1}^{\min \left\{T_{x}, R_{x}\right\}} \frac{1}{\left(K_{l}-1\right) !} G_{1,3}^{3,2}\left(\begin{array}{c|c}
1,1 & \left.\frac{K_{l} \sigma_{n}^{2}}{\bar{\beta} \bar{\lambda}_{l}}\right)
\end{array}\right.
\end{aligned}
$$

This completes the proof of Theorem 2.1. 


\section{B Proof of Lemma 2.1}

First, it is straightforward to show that the probability $\mathcal{P}_{\text {off }}^{(l)}$ is given by

$$
\mathcal{P}_{\text {off }}^{(l)}=\mathcal{P}\left\{\lambda_{l} \leq \frac{\sigma_{n}^{2}}{\bar{\beta}_{p}}\right\}=\int_{0}^{\frac{\sigma_{n}^{2}}{\beta_{p}}} f\left(\lambda_{l}\right) d \lambda_{l}=1-\frac{\Gamma\left(K_{l}, \frac{K_{l} \sigma_{n}^{2}}{\bar{\beta}_{p} \bar{\lambda}_{l}}\right)}{\left(K_{l}-1\right) !}
$$

where we used again the marginal eigenvalue pdfs $f\left(\lambda_{l}\right)$ of [88]. We proceed now with the derivation of an expression for the mean transmission opportunities number. From eqs.(2.31) and (2.45) we have

$$
\begin{aligned}
\bar{T}_{\text {opp }} & =\mathbb{E}_{\boldsymbol{\lambda}}\left\{T_{\text {opp }}\right\}=\sum_{l=1}^{\min \left\{T_{p}, R_{p}\right\}} \mathbb{E}_{\lambda_{l}}\left\{\mathbb{1}_{\left[0, \sigma_{n}^{2} / \bar{\beta}_{p}\right]}\left(\lambda_{l}\right)\right\} \\
& =\sum_{l=1}^{\min \left\{T_{p}, R_{p}\right\}} \mathcal{P}\left\{\lambda_{l} \leq \frac{\sigma_{n}^{2}}{\bar{\beta}_{p}}\right\}
\end{aligned}
$$

and therefore the proof of Lemma 2.1 is completed. 


\section{C Proof of Theorem 2.2}

Let us assume that at a fading state the WF algorithm allocates non-zero power to the last $i$ PU channels, where $0 \leq i \leq \min \left\{T_{p}, R_{p}\right\}$. Then, according to Subsection 2.3.2, where the proposed scheme for the $\mathrm{SU}$ is described, the $\mathrm{SU}$ establishes its communication link via an $\left(T_{s}-\min \left\{T_{p}, R_{p}\right\}+i\right) \times\left(R_{s}-\min \left\{T_{p}, R_{p}\right\}+i\right)$ equivalent MIMO system. Evidently, the probability $\mathcal{P}_{i}$ that the equivalent MIMO system is of $\left(T_{s}-\min \left\{T_{p}, R_{p}\right\}+i\right) \times\left(R_{s}-\min \left\{T_{p}, R_{p}\right\}+i\right)$ dimensions, equals to the probability that the WF algorithm allocates non-zero power to the $\left\{i+1, \ldots, \min \left\{T_{p}, R_{p}\right\}\right\} \mathrm{PU}$ eigen-channels and zero power to the $\{1, \ldots, i\}$ ones. By using Lemma 2.1 one can show, that the latter probability is given by

$$
\mathcal{P}_{i}=\prod_{l=1}^{i} \mathcal{P}_{o f f}^{(l)} \prod_{l=i+1}^{\min \left\{T_{p}, R_{p}\right\}}\left(1-\mathcal{P}_{o f f}^{(l)}\right)
$$

Therefore, by weighting the ergodic capacity $\bar{C}\left(L_{s}(i), \bar{\beta}_{i}, \sigma_{n}^{2}, \overline{\boldsymbol{\lambda}}_{s}(i)\right)$ of the equivalent SU MIMO system, for every possible $i$, with the corresponding probability given by eq.(2.47) and taking the sum for all possible $i, 0 \leq i \leq \min \left\{T_{p}, R_{p}\right\}$, the result of eq.(2.34) is derived and the proof is completed. 


\section{D Proof of Lemma 2.2}

In the present lemma we make the assumption that $S N R \rightarrow \infty$, which implies that the $\mathrm{SU}$ allocates equal power to its employed eigen-channels. Indeed, as $S N R \rightarrow \infty$ we have $\lambda_{k}>>\sigma_{n}^{2}$ and therefore the PU WF solution tends to that of equal power allocation one [82]. Therefore, from eq.(2.35) the interference term is given by

$$
\delta \mathbf{I}_{p}=\mathbf{U}_{p}^{H} \mathbf{H}_{s p} \delta \mathbf{Q}_{t} \hat{\mathbf{V}}_{z} \mathbf{x}_{s} \sqrt{\bar{P}_{s} /\left(L_{s}-L_{p}\right)},
$$

where $L_{s}=\min \left\{T_{s}, R_{s}\right\}$, and $L_{p}$ is the number of the employed PU eigen-channels in a fading state. Moreover, let us assume that the latter random variables are independent and that $\mathbb{E}\left\{\mathbf{x}_{s} \mathbf{x}_{s}^{H}\right\}=\mathbf{I}_{L_{s}}$. Then, by eq.(2.48) after some some calculations we have

$$
\mathbb{E}\left\{\delta \mathbf{I}_{p} \delta \mathbf{I}_{p}^{H}\right\}=\frac{\bar{P}_{s}}{L_{s}-L_{p}} \mathbf{U}_{p}^{H} \mathbf{H}_{s p} \mathbb{E}\left\{\delta \mathbf{Q}_{t} \delta \mathbf{Q}_{t}^{H}\right\} \mathbf{H}_{s p}^{H} \mathbf{U}_{p}
$$

where the orthonormal property of matrix $\hat{\mathbf{V}}_{z}$ was used. From the results of [95] and [96], where approximations of the second-order statistics concerning the estimation error of the eigen-vectors of sample covariance matrices were derived, we can compute $\mathbb{E}\left\{\delta \mathbf{q}_{i} \delta \mathbf{q}_{i}^{H}\right\}$ as

$$
\mathbb{E}\left\{\delta \mathbf{q}_{i} \delta \mathbf{q}_{i}^{H}\right\}=\frac{\sigma_{n}^{2}}{N} \sum_{k=1}^{L_{p}} \frac{\lambda_{k}+\sigma_{n}^{2}}{\lambda_{k}^{2}} \mathbf{v}_{k} \mathbf{v}_{k}^{H}
$$

where $\sigma_{n}^{2}$ is the noise variance, $N$ is the number of samples used to estimate the sample covariance matrix, $\lambda_{k}$ and $\mathbf{v}_{k}$ are the $k$-th eigenvalue and the corresponding $k-t h$ eigenvector of matrix $\mathbf{U}_{p}^{H} \mathbf{H}_{p s} \mathbf{H}_{p s}^{H} \mathbf{U}_{p}+\sigma_{n}^{2} \mathbf{I}_{R_{p}}$. Note that the approximation of $\mathbb{E}\left\{\delta \mathbf{q}_{i} \delta \mathbf{q}_{i}^{H}\right\}$ is $O\left(N^{-1}\right)$ and it is valid for large values of $N$. Now, by using eq.(2.50) we have

$$
\begin{aligned}
\mathbb{E}\left\{\delta \mathbf{Q}_{t} \delta \mathbf{Q}_{t}^{H}\right\} & =\mathbb{E}\left\{\delta \mathbf{q}_{1} \delta \mathbf{q}_{1}^{H}\right\}+\cdots+\mathbb{E}\left\{\delta \mathbf{q}_{\left(T_{s}-L\right)} \delta \mathbf{q}_{\left(T_{s}-L\right)}^{H}\right\} \\
& =\frac{\left(L_{s}-L_{p}\right) \sigma_{n}^{2}}{N} \sum_{k=1}^{L_{p}} \frac{\lambda_{k}+\sigma_{n}^{2}}{\lambda_{k}^{2}} \mathbf{v}_{k} \mathbf{v}_{k}^{H}
\end{aligned}
$$




$$
=\frac{\left(L_{s}-L_{p}\right) \sigma_{n}^{2}}{N} \mathbf{V}_{D_{p s}} \tilde{\Lambda}_{D_{p s}} \mathbf{V}_{D_{p s}}^{H}
$$

where $\mathbf{D}_{p s}=\mathbf{U}_{p}^{H}\left(1: R_{p}, 1: L_{p}\right) \mathbf{H}_{p s}=\mathbf{U}_{D_{p s}} \boldsymbol{\Sigma}_{D_{p s}} \mathbf{V}_{D_{p s}}^{H}$. Now, by plugging the previous result into eq.(2.49) and using the aforementioned SVD of matrix $\mathbf{D}_{p s}$ we have

$$
\mathbb{E}\left\{\delta \mathbf{I}_{p} \delta \mathbf{I}_{p}^{H}\right\}=\frac{\bar{P}_{s} \sigma_{n}^{2}}{N} \mathbf{U}_{D_{p s}} \tilde{\boldsymbol{\Lambda}}_{D_{p s}} \boldsymbol{\Lambda}_{D_{p s}} \mathbf{U}_{D_{p s}}^{H}
$$

From the previous equation, it easy now to see that

$$
\mathbb{E}\left\{\left\|\mathbf{I}_{p}\right\|^{2}\right\}=\frac{\bar{P}_{s} \sigma_{n}^{2}}{N}\left(L_{p}+\sum_{k=1}^{L_{p}} \frac{\sigma_{n}^{2}}{\lambda_{k}}\right)
$$

Now, as it was also stated above, as $S N R \rightarrow \infty$ we have $\lambda_{k}>>\sigma_{n}^{2}$ and $L_{p}=$ $\min \left\{T_{p}, R_{p}\right\}$, since the PU WF solution tends to the equal power allocation one. Therefore, the previous equation can be written as

$$
\mathbb{E}\left\{\left\|\mathbf{I}_{p}\right\|^{2}\right\} \approx \frac{\bar{P}_{s} L_{p} \sigma_{n}^{2}}{N}
$$

and this concludes the proof of Lemma 2 . 


\section{Chapter 3}

\section{Decentralized Adaptive}

Eigenvalue-Based Spectrum

Sensing for Multi-antenna

\section{Cognitive Radio Systems}

\subsection{Introduction}

In section 1.4 the concept of spectrum sensing was briefly introduced. Among the different approaches presented in that chapter, the Eigenvalue - Based Spectrum Sensing Techniques (EBSS) [18]-[19] were highlighted due to they desired characteristics: the fact that they can be implemented in a completely blind manner and the improved performance they present for specific signal categories. However, those features come at the expense of high complexity since they require the EigenValue Decomposition (EVD) of the received signal's sample covariance matrix. Moreover, the computation of the involved decision thresholds is generally based on the asymptotic (limiting) distributions of the corresponding test statistics and requires a large number of samples in order to attain a satisfactory performance. Recently, close approximations for the distribution functions of the test statistics have been proposed in the literature, [27] 
[97], though they have quite a complex form. Thus, the computation of the decision thresholds is actually performed by numerical methods which increase even further the computational complexity.

The high complexity required for implementing the batch EBSS techniques makes them impractical for cases where time-varying channels are involved and/or continuous monitoring of a specific spectrum band is required (dual radio approach - Section 1.4). That is, in cases where the involved channels are varying within the sensing period, the batch techniques exhibit performance degradation if the length of the sensing period is too large compared to channels' coherence time. For example, severe deep fading events within the sensing period, could lead to an increased number of PU miss-detection events. Therefore the batch EBSS techniques, for different reasons, are inappropriate for the sensing architectures discussed previously in this section (i.e., single radio and dual radio). Indeed, they are not suitable for single radio sensing due to their lacking of tracking abilities, and also they are not a viable solution for dual radio sensing due to the prohibitive complexity of continuous monitoring.

Apart from their high complexity, the existing EBSS techniques, to the best of our knowledge, are all centralized based approaches exhibiting the limitations that were referenced in Section 1.4. Therefore, the main goal here is to develop cooperative adaptive EBSS techniques that function in a completely decentralized manner and not exhibit the previously discussed limitations. It is noteworthy to mention, that a constituent part of the new cooperative EBSS technique is a new distributed subspace tracking algorithm which can be considered as a by-product of the present work. Note that little work has been published so far for the problem of distributed subspace tracking [98]-[99]. The existing approaches are suitable for large sensor networks rather than small scale topologies of adjacent SU nodes as it is typically assumed in cooperative spectrum sensing scenarios. Moreover, the existing approaches present slow convergence speed and bad performance since they do not guarantee the orthonormality of the tracked eigenvectors (see also Section 3.6.1). As it will be shown later the distributed subspace tracking algorithm does not suffer from the above limitations. 
More specifically, the contributions to research field are the following ones [72][73]. At first, novel single SU (non cooperative) adaptive EBSS techniques are derived based on well-known subspace tracking methods. Note that, to the best of our knowledge, this is the first time that adaptive versions of the EBSS techniques are derived, even for the non-cooperative case. Then, the distributions of the adaptive test-statistics are derived in order to compute the required decisions thresholds. It turns out that accurate approximations of the test statistics correspond to well-known tabulated functions improving further the practicality of the new adaptive schemes over the batch ones. Then, a novel cooperative adaptive EBSS method is proposed by first developing a novel distributed adaptive subspace tracking algorithm. The proposed adaptive EBSS and cooperative adaptive EBSS methods are compared to the corresponding batch EBSS approaches, in terms of performance, for static and timevarying channels and for single (fixed spectrum sensing time) and double (continuous spectrum monitoring) radio architectures.

The rest of the chapter is organized as follows. In Section 3.2 the system description is provided. In Section 3.3 a brief description is given for the EBSS techniques considered in the present paper. In Section 3.4, adaptive versions of the EBSS (ADEBSS) techniques are derived. In Section 3.5, the test statistics' distribution functions of the proposed AD-EBSS techniques and the corresponding decision thresholds are derived In Section 3.6, the cooperative EBSS scheme is presented. Section 3.7 presents the simulations, and Section 3.8 concludes the chapter.

\subsection{System Description}

Let us assume that a single-antenna PU node and $K \mathrm{SU}$ nodes of $R_{x}$ antennas each one, are operating in the same frequency band in a typical interweave CR scheme (Section 1.3.3). The system description is given for the single radio approach, though it can be appropriately modified so as to be applied to the double radio one. Thus, the time axis is assumed to be divided into transmit time intervals (time-slots). The time-slot is considered as the basic unit of time scheduling. At the beginning of each 
SU time-slot, the SUs sense the frequency band so as to determine if it is occupied by a PU transmission. Let us also assume that during each sensing period, each one of the $K$ SUs collects $N$ sample vectors $\mathbf{y}_{n}^{i}, 1 \leq n \leq N$ and $1 \leq i \leq K$, of dimensions $1 \times R_{x}$. In a centralized approach, a node that plays the role of the fusion center receives from the SUs all collected vector samples. Then, the fusion center processes the samples jointly and decides if active PU transmissions exist in the environment and notifies accordingly the SU nodes. More specifically, in the associated cooperative spectrum sensing problem the following hypothesis test is considered at the fusion center,

$$
\begin{aligned}
& \mathcal{H}_{0}: \mathbf{y}_{n}=\mathbf{z}_{n} \\
& \mathcal{H}_{1}: \mathbf{y}_{n}=\mathbf{h} x_{n}+\mathbf{z}_{n},
\end{aligned}
$$

where $\mathbf{y}_{n}=\left[\mathbf{y}_{n}^{1}, \ldots, \mathbf{y}_{n}^{K}\right]^{T}, \mathbf{z}_{n}$ is an $K R_{x} \times 1$ additive noise random variable modeled as $\mathcal{C N}\left(0, \sigma_{z}^{2}\right), \mathbf{h}$ is a $K R_{x} \times 1$ vector that contains all complex flat channel gains corresponding to the links between the PU and the antennas of all SUs, and $x_{n}$ is the transmitted PU symbol. That is, under the hypothesis $\mathcal{H}_{0}$, the PU is idle and the signals $\mathbf{y}_{n}^{i}$ received at the SUs contain only noise. On the other hand, under the hypothesis $\mathcal{H}_{1}$, the PU transmits data and the received SUs' signals are a superposition of these data (scaled by the channel gains) and noise. An EBSS technique decides between the two hypotheses by employing test statistics that are functions of the eigenvalues of the received signals' covariance matrix. In the following section a brief description of the existing EBSS techniques is given.

\subsection{Centralized Batch Cooperative Eigenvalue-Based Spectrum Sensing}

In literature so far, only batch EBSS techniques have been considered, in the sense that the derivation of the test statistics is based on the EVD of the sample covariance matrix formed by a number of $N$ received vectors. Moreover, only centralized cooperative extensions of the EBBS techniques have been proposed so far. As long as a 
fusion center exists to collect the $\mathrm{SU}$ vector samples $\mathbf{y}_{n}^{i}$ in the cooperative case, the application of a batch EBSS technique is almost identical in both the non-cooperative (single) SU and the cooperative cases.

We now provide a brief description of the existing approaches for the cooperative case, though they are directly applicable to the non-cooperative case by setting the number of SU nodes equal to $K=1$. We consider that the fusion center receives the SUs' sample vectors $\mathbf{y}_{n}^{i}$ through noise-free communication links and forms the aggregate vector $\mathbf{y}_{n}=\left[\mathbf{y}_{n}^{1}, \ldots, \mathbf{y}_{n}^{K}\right]^{T}$ as discussed in the previous section. The covariance matrix $\mathbf{R}$ of vector $\mathbf{y}_{n}$, under the two hypotheses, is given by,

$$
\mathbf{R}=\mathbb{E}\left\{\mathbf{y}_{n} \mathbf{y}_{n}^{H}\right\}=\left\{\begin{array}{cc}
\sigma_{z}^{2} \mathbf{I}_{R_{x}} & \mathcal{H}_{0} \\
\sigma_{x}^{2} \mathbf{h h}^{H}+\sigma_{z}^{2} \mathbf{I}_{R_{x}} & \mathcal{H}_{1}
\end{array}\right.
$$

where $\mathbb{E}(\cdot)$ denotes the expected value operator, $\sigma_{x}^{2}$ is the PU's transmitted signal's variance and $(\cdot)^{H}$ denotes the Hermitian of a matrix. Let us also denote as $\lambda_{1} \geq$ $\cdots \geq \lambda_{R_{x}}$ the ordered eigenvalues of matrix $\mathbf{R}$. Under the hypothesis $\mathcal{H}_{0}$, all the latter eigenvalues are due to noise only, that is, $\lambda_{1}=\cdots=\lambda_{R_{x}}=\sigma_{z}^{2}$. Under the hypothesis $\mathcal{H}_{1}$, the first eigenvalue is given by $\lambda_{1}=\sigma_{1}^{2}+\sigma_{z}^{2}$, where $\sigma_{1}^{2}$ is the received PU signal's noise power and the rest eigenvalues are given by $\lambda_{2}=\cdots=\lambda_{R_{x}}=\sigma_{z}^{2}$. It is clear that the eigenvalues of the covariance matrix can be used to form a sufficient test statistics for the spectrum sensing problem. In the following equations three different test statistics are defined.

$$
\begin{aligned}
T^{M E D}\left(\lambda_{1}\right) & =\frac{\lambda_{1}}{\sigma_{z}^{2}}, \\
T^{G L R T}\left(\lambda_{1}, \ldots, \lambda_{R_{x}}\right) & =\frac{\lambda_{1}}{\frac{1}{N-1} \sum_{m=2}^{R_{x}} \lambda_{m}}, \\
T^{M M E D}\left(\lambda_{1}, \lambda_{R_{x}}\right) & =\frac{\lambda_{1}}{\lambda_{R_{x}}} .
\end{aligned}
$$

If the test-statistics' value is above a predefined threshold, a decision in favor of hypothesis $\mathcal{H}_{1}$ is taken and vice versa. The test statistic $T^{M E D}$ is commonly known as the Maximum Eigenvalue Detector and it is proved to be the Generalized Likelihood 
Ratio Test (GLRT) for the hypothesis testing of Eqs.(3.1)-(3.2) under the assumption that only the noise variance is known to the SU (i.e., $\mathbf{h}$ and $\sigma_{x}^{2}$ are unknown) [100]. The test statistic $T^{G L R T}$ is proved to be the GLRT for the previous hypothesis testing without assuming that the noise variance is known to the SU [100], [101], [102]. Finally, the $T^{M M E D}$ test statistic is commonly known as the Maximum-Minimum Eigenvalue detector and it has obviously a simpler form as compared to $T^{G L R T}$.

In practice, the fusion center (or a single $\mathrm{SU}$ ) computes the sample covariance matrix of the received signals, which is given by,

$$
\hat{\mathbf{R}}=\frac{1}{N} \sum_{i=1}^{N} \mathbf{y}_{n} \mathbf{y}_{n}^{H} .
$$

The noise eigenvalues of the sample covariance matrix are random variables due to the finite number of samples that are used for its computation. The distribution of these noise eigenvalues is used to compute the decision threshold in a NeymanPearson sense for a pre-defined probability of false alarm. Detailed information on how to compute the decision thresholds is given in [18], [19] and [100].

We can now proceed with the development of the cooperative adaptive EBSS methods. For clarity purposes, we first deal with the non-cooperative case in which only a single SU is involved. Then the extension of the proposed adaptive approaches to the cooperative case is derived.

\subsection{Adaptive Eigenvalue-Based Spectrum Sensing for the Single SU Case}

As already mentioned in the Section 3.1, the batch EBBS techniques of the previous section are suiTable 3-In cases where constant channels during the timeslots and single radio architectures are assumed. However, if the channels are varying within the timeslot, as it is the case with fading channels, the EBBS techniques are impractical to use due to high complexity (of the regularly required EVD computation) and the fact that they demand a large number of samples as discussed in the Introduction. 
Indeed, within the sensing period, there is high probability of a deep fading event that could degrade the performance of the EBSS techniques. Therefore, the spectrum sensing techniques must be applied more than once within each PU time slot, in order to track the channel variations and avoid a PU "miss-detection" when a deep fading event occurs. Moreover, as again discussed in the Introduction, the complexity of the EBSS techniques is prohibitive for dual radio architectures where continuous spectrum monitoring is to be applied.

On-line implementations of the EBSS techniques, capable of tracking the fading channels' variations, can be derived by using low-complexity subspace tracking (ST) algorithms. Several ST algorithms have been proposed in the literature over the past years, in the context of other applications (e.g. Direction of Arrivals estimation and tracking), which are able to track with linear complexity the desired subset of the subspace of a covariance matrix.

In this paper the complex version of the Fast Data Projection Method (FDPM) is employed [93]. The FDPM steps are summarized in the following equations.

$$
\begin{aligned}
\mathbf{r}_{n} & =\mathbf{U}_{n-1}^{H} \mathbf{y}_{n} \\
\mathbf{U}_{n} & =\mathbf{U}_{n-1} \pm \mu \mathbf{y}_{n} \mathbf{r}_{n}^{H} \\
\mathbf{U}_{n} & =\operatorname{orth}\left\{\mathbf{U}_{n}\right\} \\
\boldsymbol{\Lambda}_{n} & =\alpha \boldsymbol{\Lambda}_{n-1}+(1-\alpha)\left|\mathbf{r}_{n}\right|^{2},
\end{aligned}
$$

where $\mathbf{U}_{n}$ and $\boldsymbol{\Lambda}_{n}$ are the $R_{x} \times L$ matrix and $L \times 1$ vector that contain the $L$ principal (minor) eigenvectors and eigenvalues, respectively, and $\alpha, \mu$ are step size parameters. Note that in equation $(3.9)$ the sign is a $(+)$ or $(-)$ depending on whether the signal or the noise subspace, respectively, is updated. Eq.(3.11) tracks the corresponding eigenvalues. An orthonormalization procedure is applied in Eq.(3.10), based on a low complexity Householder transformation [93], given by the following equations

$$
\begin{aligned}
\mathbf{a}_{n} & =\mathbf{r}_{n}-\left\|\mathbf{r}_{n}\right\| e^{j\left(\operatorname{angle}\left(\mathbf{e}_{1}^{H} \mathbf{y}_{n}\right)\right)} \mathbf{e}_{1} \\
\mathbf{U}_{n} & =\mathbf{U}_{n}-\frac{2}{\left\|\mathbf{a}_{n}\right\|^{2}}\left(\mathbf{U}_{n} \mathbf{a}_{n}\right) \mathbf{a}_{n}^{H}
\end{aligned}
$$




$$
\mathbf{U}_{n}=\operatorname{norm}\left\{\mathbf{U}_{n}\right\}
$$

In eq.(3.12) $\mathbf{e}_{1}=[1,0 \ldots 0,0]$ and in eq.(3.13), norm $\{\cdot\}$ denotes the operator that normalizes the columns of a matrix. For the MED test statistic, only the first (maximum) eigenvalue is required, so $\mathbf{U}_{n}$ is a $R_{x} \times 1$ matrix and $\boldsymbol{\Lambda}_{n}$ is a scalar that contains the current estimate of the maximum eigenvalue. The orthonormalization step is also reduced to a simple normalization one, i.e. $\mathbf{U}_{n}=\mathbf{U}_{n} /\left\|\mathbf{U}_{n}\right\|$. In a similar way, the MMED test statistic is estimated. At first, the signal subspace version of the FDPM is employed (with $(+)$ in $(3.9)$ ) to track the maximum eigenvalue and then the noise subspace version follows (with (-) in (3.9)) to track the minimum one. For the GLRT test statistic the complete FDPM (eqs.(3.8)-(3.11)) is used since all the $R_{x}$ eigenvalues of $\hat{\mathbf{R}}_{n}$ are required in eq.(3.5).

It is evident now that, by employing the FDPM method, the SU is capable of tracking, with low complexity, the value of any of the test-statistics under consideration at every time index within the sensing period. Therefore, once a new signal vector is received, the $\mathrm{SU}$ updates the employed test-statistic and decides if a change in the state has been occurred (from $\mathcal{H}_{0}$ to $\mathcal{H}_{1}$ and vice-versa).

Let us now elaborate on how the adaptive EBSS techniques are specifically applied to single and dual radio architectures. At first the single radio approach is considered. In this case, the adaptive EBSS techniques are applied within a fixed sensing period and the final decision is taken at the end based on the number of $\mathcal{H}_{1}$ decisions within the sensing period. That is, if a PU signal is detected for a percentage $p$ of the sensing period then the existence of a PU signal is decided. The percentage $p$ is used to calibrate the $\mathcal{P}_{f}$ to a desired value. An increase in the value of $p$ results in a decrease of $\mathcal{P}_{f}$ and $\mathcal{P}_{d}$ and vice versa. The latter approach improves the performance in cases where time-varying channels are involved. Let us now consider to the dual radio approach where continuous spectrum monitoring is applied. Here, the SU employs the algorithm of Table 3-I via which the required continuous spectrum monitoring is implemented. According to the aforementioned algorithm, the SU updates continuously the value of the corresponding test statistics and decides that a change 
Table 3-I: Adaptive EBSS for the single SU case

\section{Initialization:}

The SU detects the present state $\left(\mathcal{H}_{0}\right.$ or $\left.\mathcal{H}_{1}\right)$ by applying the adaptive EBSS technique for a sufficient number of received signals, until an initial decision is taken.

for $i=n \rightarrow \infty$ do

Update the employed Test-Statistic $T$ via the FDPM (3.8)-(3.11)

if $T \geq \eta, \forall n \in\left[n-N_{c}, n\right]$ under $\mathcal{H}_{0} \| T<\eta, \forall n \in[n-T c, n]$ under $\mathcal{H}_{1}$ then

Raise an alarm, change in the state has been detected;

end if

end for

in the state has occurred if the test statistic changes behaviour for a number of $N_{c}$ consecutive received signals. The parameter $N_{c}$ is used for calibrating the rate of false alarms and miss-detection events to desired values. A small value of $N_{c}$ results in small detection delays and increased rates of false alarm to abrupt changes in the PU behaviour and vice-versa.

\subsection{Test Statistics Distributions and Decision Thresh- olds}

In this section, the Cumulative Distribution Functions (CDF) of the three test statistics of Eqs.(3.4)-(3.6) are derived for the adaptive case, under the hypothesis $\mathcal{H}_{0}$, i.e., when no information signal is present in the signal received by the SU. Based on eq.(3.11) of the FDPM, the distribution functions of the involved test statistics can be tracked at every time index $n$. The following lemma provide expressions for the distribution functions of the corresponding test statistics.

Lemma 3.3 The distribution functions of the adaptive MED test-statistics updated by eq.(3.11) for a $S U$ of $R_{x}$ antennas under the hypothesis $\mathcal{H}_{0}$ can be approximated by the functions,

$$
\begin{aligned}
F_{T^{M E D}}(x ; \xi, \rho) & =\frac{\gamma(\xi x, \rho)}{\Gamma(\rho)}, \\
F_{T^{G L R T}}\left(x ; \rho, R_{x}\right) & =\mathcal{I}_{\frac{\left(R_{x}-1\right) x}{x+1}}\left(\rho,\left(R_{x}-1\right) \rho\right),
\end{aligned}
$$




$$
F_{T^{M M E D}}(x ; \rho)=\mathcal{I}_{\frac{x}{x+1}}(\rho, \rho)
$$

respectively, where $\xi=\frac{\left(1-\alpha^{n}\right)(1+\alpha)}{\left((1-\alpha)\left(1-\alpha^{2 n}\right)\right)}, \rho=\frac{\left(1-\alpha^{n}\right)^{2}(1+\alpha)}{\left((1-\alpha)\left(1-\alpha^{2 n}\right)\right)}, \gamma(x, \rho)=\int_{0}^{x} t^{\rho-1} e^{-t} d t$ is the lower incomplete gamma function, $\Gamma(\rho)=\int_{0}^{\infty} t^{\rho-1} e^{-t} d t$ denotes the ordinary gamma function and $\mathcal{I}_{x}\left(\rho_{1}, \rho_{2}\right)=\int_{0}^{x} t^{\rho_{1}-1}(1-t)^{\rho_{2}-1} d t$ is the incomplete beta function.

Proof: Observe that, under hypothesis $\mathcal{H}_{0}$, the signal vector $\mathbf{y}_{n}$ received by the SU consists of i.i.d. complex Gaussian noise samples $\mathcal{C N}\left(0, \sigma_{z}^{2}\right)$. Since matrix $\mathbf{U}_{n-1}$ is orthonormal, the entries $\mathbf{r}_{n}^{(i)}$ of vector $\mathbf{r}_{n}=\mathbf{U}_{n-1}^{H} \mathbf{y}_{n}$ are also i.i.d. $\mathcal{C N}\left(0, \sigma_{r}^{2}\right)$. From eq.(3.11), the $l$-th eigenvalue of the covariance matrix is estimated as,

$$
\lambda_{l}(n)=\sum_{i=0}^{n} \alpha^{i}(1-\alpha)\left|\mathbf{r}_{n}^{(i)}\right|^{2} .
$$

According to the previous equation, the MED test statistic can be expressed as a weighted sum of chi-squared variables with each one derived by squaring the absolute value of a random variable $\phi_{i} \sim \mathcal{C N}(0,1)$. A close approximation to the previous distribution can be derived by properly applying the results of [103] to our case. Let us consider the following RV

$$
T \triangleq \sum_{i=1}^{m} w_{i} \zeta_{i}^{2}
$$

where $\zeta_{i} \sim \mathcal{N}(0,1)$ and $w_{i} \in \mathbb{R}$. Welch, in a 1938 paper [103], proposed an approximation of the distribution of variable $T$ by a scaled chi-squared distribution of $\rho$ degrees of freedom. That is, $T \sim \frac{1}{\xi} \chi_{\rho}^{2}$, where $\xi=\frac{\sum_{i=1}^{m} w_{i}}{\sum_{i=1}^{m} w_{i}^{2}}, \xi=\frac{\left(\sum_{i=1}^{m} w_{i}\right)^{2}}{\sum_{i=1}^{m} w_{i}^{2}}$ and the corresponding CDF is given by

$$
F_{T}(x ; \xi, \rho)=\frac{\gamma(\xi / 2 x, \rho / 2)}{\Gamma(\rho / 2)} .
$$

Now, by defining $w_{i}=\alpha^{i}(1-\alpha) / 2$ and using eq.(3.20) in the case of complex normal variables $\phi_{i}$, we can apply the previous results to the MED test statistic (3.4). Moreover, observe that the weights $w_{i}$ are actually terms of a geometric sequence enabling as to compute closed forms for the parameters $\rho$ and $\xi$. Thus, it can be easily verified that $\xi=\frac{2\left(1-\alpha^{n}\right)(1+\alpha)}{\left((1-\alpha)\left(1-\alpha^{2 n}\right)\right)}$ and $\rho=\frac{2\left(1-\alpha^{n}\right)^{2}(1+\alpha)}{\left((1-\alpha)\left(1-\alpha^{2 n}\right)\right)}$. Finally, by combining these expressions 
with eq.(3.20) the proof for MED is completed.

In the case of the MMED test statistic, we seek for the distribution of the ratio of two eigenvalues of matrix $\hat{\mathbf{R}}_{n}$, that are estimated via Eq.(3.11). The required distribution is equivalent to the distribution of the ratio $\frac{T^{M E D}\left(\lambda_{1}\right)}{T^{M E D}\left(\lambda_{R_{x}}\right)}$. Therefore, the corresponding CDF is equal to the one of the ratio of two independent $T^{M E D}$ random variables. It turns out that the distribution of the MMED statistic can be approximated by the beta prime distribution with the corresponding CDF given by eq.(3.17). The detailed proof is given at Appendix 3.A.

Finally, the CDF of the GLRT test statistic can be computed by firstly observing that the random variable $\sum_{m=2}^{R_{x}} \lambda_{m} \sim \frac{1}{\xi} \chi_{\left(R_{x}-1\right) \rho}^{2}$, where the parameters $\xi$ and $\rho$ are defined as in the case of the MED distribution. Then the CDF of the ratio of the two independent random variables $\frac{\lambda_{1}}{\sum_{m=2}^{R} \lambda_{m}}$ is computed in a similar way to the MMED case. As long as the CDF of the ratio under consideration is computed, it is easy then to compute the CDF of the scaled random variable $T^{G L R T}=\frac{\lambda_{1}}{\frac{1}{R_{x}-1} \sum_{m=2}^{R_{x}} \lambda_{m}}$ which is given by eq.(3.16).

As it can be seen from the results of Lemma 3.1, the CDFs of the adaptive test statistics involve the computation of well-known tabulated functions and the same comment is true for their corresponding inverse functions. The latter observation enables the SU to easily compute the decision thresholds in a Neyman-Pearson sense, for a pre-defined probability of false alarm $\mathcal{P}_{f}$, as opposed to the case of the batch test statistics where usually numerical methods are required. At first, the decision threshold $\eta^{M E D}$ for the $M E D$ test statistic is computed. We equivalently have

$$
\begin{aligned}
& P_{f}=P\left\{T^{M E D}>\eta^{M E D} \mid \mathcal{H}_{0}\right\}=1-F_{T^{M E D}}(x ; \xi, \rho) \Rightarrow \\
& \eta^{M E D}=F_{T^{M E D}}^{-1}\left(1-P_{f} ; \xi, \rho\right) .
\end{aligned}
$$

The decision thresholds for the adaptive MMED and GLRT test statistics can be computed in a similar way, and are given by the following equations,

$$
\eta^{M M E D}=\frac{\mathcal{I}^{-1}\left(1-P_{f} ; \rho, \rho\right)}{1-\mathcal{I}^{-1}\left(1-P_{f} ; \rho, \rho\right)}
$$




$$
\eta^{G L R T}=\frac{\mathcal{I}^{-1}\left(1-P_{f} ; \rho,\left(R_{x}-1\right) \rho\right)}{\left(R_{x}-1\right)-\mathcal{I}^{-1}\left(1-P_{f} ; \rho,\left(R_{x}-1\right) \rho\right)}
$$

\subsection{Cooperative Decentralized Adaptive Eigenvalue- Based Spectrum Sensing}

In this section, the previously derived adaptive EBSS technique is extended to the cooperative case, in which multiple SUs sense the spectrum in a collaborative manner. The new distributed technique is designed so as, a) to improve the sensing performance by forming a virtual multiple antenna system that performs a joint EBSS technique, b) to distribute the computation overhead among the SU nodes, and c) to enable each user to track the joint test statistics at each time index within the sensing period so as to reach a common decision in a decentralized manner. Apart from the benefits of a decentralized approach discussed in the Introduction, the latter feature is crucial in scenarios where the multiple nodes employ a cooperative transmission scheme (e.g. cooperative beamforming [50]), and thus all the involved nodes should reach a common decision concerning the PU existence. Clearly, the proposed decentralized adaptive EBSS methods require the development of a distributed adaptive subspace tracking method in order to track the corresponding test statistics of eqs.(3.4) - (3.6). Therefore, a corresponding novel distributed subspace tracking scheme is first developed by extending the FDPM of [93]. The present section is divided into three subsections. Subsection 3.6.1 provides a brief discussion on distributed subspace tracking and the related work that has published in literature. In Subsection 3.6.2 the proposed distributed subspace tracking method is described. Finally, in Subsection 3.6.3, the cooperative EBSS methods are described.

\subsubsection{Related Work}

To the best of our knowledge, little work has been published in the literature concerning the problem of distributed subspace tracking. The authors in [98] develop a 
diffusion based technique of OJA rule in which several subspace tracking methods are based. In [99] a distributed version of the well-known PAST technique is presented. The previous methods are more suitable for ad hoc wireless sensor networks with a large number of nodes since they are based on diffusion based strategies. Since in the scenario studied in this paper, the number of nodes that are involved in the network topology is relative small, we seek for suitable distributed strategies that minimize the required communication overhead. Moreover, the approaches of [98] and [99] provide estimations for the eigenvectors only and not for the corresponding eigenvalues. Also, they converge rather slowly since they are gradient flow based approaches [93], and finally they do not guarantee the orthogonality of the estimated eigenvectors resulting in severe performance degradation.

In the following, a novel approach is proposed for solving the distributed subspace tracking problem by extending the FDPM approach [93]. The new approach is based on an incremental strategy that requires no fusion center, it exhibits minimum communication overhead [104] and distributes evenly the computation cost among the SU nodes, thus enabling each one of them to perform the spectrum sensing test at every time index within the sensing period. Moreover, it converges faster since the Data Projection Method (DPM) is based on the power method [105] and guarantees the orthogonality of the tracked eigenvectors. Note, that most of the cooperative spectrum sensing methods proposed in literatue are fusion center based, hence they exhibit high networking cost and create a computational and storage bottleneck for the whole system. In the following subsection the new distributed subspace tracking scheme is derived.

\subsubsection{Distributed Data Projection Method (DDPM)}

Let us assume that $K \mathrm{SU}$ nodes form a network where the $i$-th node is connected via direct links only to the $(i-1)$-th and the $(i+1)$-th nodes. The communication links are assumed to be established via ideal (noise free) channels. Note that

the latter assumption is typical in both the distributed adaptive signal processing [104] and cooperative spectrum sensing literature [22] - [26]. For simplicity, we as- 
sume that each SU node has the same number of $R_{x}$ antennas, though the results are directly applicable in cases where the nodes may have different numbers of antennas. At each time index of the sensing period, the $i$-th $\mathrm{SU}$ obtains a $R_{x} \times 1$ vector of samples $\mathbf{y}_{n}^{i}$. Let us now assume that we are interested in tracking the $L$ first principal (or minor) components of the correlation matrix $\mathbf{R}$ of the aggregate vector $\left[\mathbf{y}_{n}^{1}, \ldots, \mathbf{y}_{n}^{K}\right]^{T}$. The task of tracking each of the $L$ eigenvectors is properly distributed to the $\mathrm{SU}$ nodes in a way that the $i$-th node tracks the elements of the sub-matrix $\mathbf{U}_{n}\left((i-1) R_{x}+1: i R_{x}, 1: L\right)$ of the aggregate eigenvectors matrix $\mathbf{U}_{n}$. For simplicity, the later sub-matrix will be denoted by $\mathbf{U}_{n}^{i}$. Thus, each node needs to update only a specific part of the subspace. Moreover, each node is capable of tracking the corresponding $L$ principal (minor) eigenvalues of the aggregate system, denoted by $\boldsymbol{\Lambda}_{n}^{i}$. As explained below, at each time index $n$, the DDPM requires three spatial iterations in order to update the eigenvectors and eigenvalues matrices.

By observing eq.(3.9), the subspace update can be done in a local computation step at node $i$ given by

$$
\mathbf{U}_{n}^{i}=\mathbf{U}_{n-1}^{i} \pm \mu \mathbf{y}_{n}^{i} \mathbf{r}_{n}^{H}
$$

Now, in order to execute the local update step of eq.(3.24) each node must know the value of the $\mathbf{r}_{n}$. Observe that the computation of $\mathbf{r}_{n}$ requires a spatial iteration in which the $i$-th node performs the local computation of eq.(3.25) and sends the quantity $\mathbf{r}_{l o c}^{i}$ to the $(i+1)$-th node. That is,

$$
\mathbf{r}_{l o c}^{i}=\mathbf{r}_{l o c}^{i-1}+\mathbf{U}_{n-1}^{*^{i}} \mathbf{y}_{n}^{i}
$$

Thus, starting from the 1st node the variable $\mathbf{r}_{l o c}^{i}$ is updated in an incremental manner and at the end of this first spatial iteration the $K$-th node has the exact value of $\mathbf{r}_{n}$ given by

$$
\mathbf{r}_{n}=\sum_{i=1}^{K} \mathbf{r}_{l o c}^{i}
$$

The second spatial iteration starts from the last node $K$ of the network. As it was mentioned the $K$-th node has already the exact value of $\mathbf{r}_{n}$ eq.(3.26) and therefore 
Table 3-II: The DDPM Algorithm

1: Initialization: $\mathbf{U}_{0} \leftarrow \mathbf{I}_{R_{x} \times L} \& \boldsymbol{\Lambda}_{0} \leftarrow \mathbf{0}_{L \times 1}$

2: Previous Instant Data: Node $i$ has $\mathbf{U}_{n-1}^{i}$ and $\boldsymbol{\Lambda}_{n-1}^{i}$

3: New Data: Node $i$ has a new vector sample $\mathbf{y}_{n}^{i}$

4: Apply:

5: 1st Spatial Iteration:

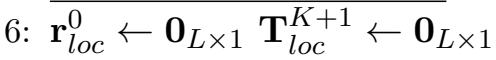

7: for $i=1 \rightarrow K$ do

8: $\quad \mathbf{r}_{l o c}^{i} \leftarrow \mathbf{r}_{l o c}^{i-1}+\mathbf{U}_{n-1}^{i^{*}} \mathbf{y}_{n}(i) \quad \%$ Incremental Comp. of $\mathbf{r}_{n}$

9: end for

10: 2nd Spatial Iteration:

11: for $i=K \rightarrow 1$ do

12: $\quad \boldsymbol{\Lambda}_{n}^{i}=\alpha \boldsymbol{\Lambda}_{n-1}^{i}+(1-\alpha)\left|\mathbf{r}_{n}\right|^{2}, \quad \%$ Update of $\boldsymbol{\Lambda}_{n}^{i}$

13: $\quad \mathbf{U}_{n}^{i} \leftarrow \mathbf{U}_{n-1}^{i} \pm \mu \mathbf{y}_{n}^{i} \mathbf{r}_{n}^{H} \quad \%$ Update of $\mathbf{U}_{n}^{i}$

14: $\quad \mathbf{a}_{n} \leftarrow \mathbf{r}_{n}-\left\|\mathbf{r}_{n}\right\| \mathbf{e}_{1} \quad \%$ Distributed Orthogonalization of $\mathbf{U}_{n}$

15: $\quad \mathbf{U}_{n}^{i} \leftarrow \mathbf{U}_{n}^{i}-\frac{2}{\left\|\mathbf{a}_{n}\right\|^{2}}\left(\mathbf{U}_{n}^{i} \mathbf{a}_{n}\right) \mathbf{a}_{n}^{H}$

16: $\quad \mathbf{T}_{l o c}^{i} \leftarrow \mathbf{T}_{l o c}^{i-1}+\sum_{l=1}^{L}\left|\mathbf{U}_{n}^{j}(:, l)\right|^{2} \quad \%$ Incr. Comp. of $\mathbf{T}_{n}$

17: end for

18: 3rd Spatial Iteration:

19: for $i=1 \rightarrow K$ do

20: $\quad \mathbf{U}_{n}^{i} \leftarrow \operatorname{diag}\left(\mathbf{T}_{n}\right)^{1 / 2} \mathbf{U}_{n}^{i} \quad \%$ Distr. Normalization of $\mathbf{U}_{n}$

21: end for

it is able to update the corresponding sub-matrix of eigenvectors as determined by eq.(3.24), then update the eigenvalues by using eq.(3.11) locally and apply the orthogonalization transformation to the updated eigenvectors sub-matrix, that is

$$
\mathbf{U}_{n}^{i}=\mathbf{U}_{n}^{i}-\frac{2}{\left\|\mathbf{a}_{n}\right\|^{2}}\left(\mathbf{U}_{n}^{i} \mathbf{a}_{n}\right) \mathbf{a}_{n}^{H}
$$

where the quantity $\mathbf{a}_{n}$ is computed by applying eq.(3.12) locally. Clearly, in order to complete the eigenvectors update, the normalization step of eq.(3.13) is required. Therefore, the last local computation of the $i-t h$ node at the 2 nd spatial iteration is given by

$$
\mathbf{T}_{l o c}^{i}=\mathbf{T}_{l o c}^{i-1}+\sum_{l=1}^{L}\left|\mathbf{U}_{n}^{j}(:, l)\right|^{2}
$$

Now observe that, by incrementally applying eq.(3.28) at the end of the 2nd spatial iteration the 1 st node has the vector $\mathbf{T}_{n}=\left[\left\|\mathbf{U}_{n}(:, l)\right\|^{2}, \ldots,\left\|\mathbf{U}_{n}(:, L)\right\|^{2}\right]$ whose $i$-th element is the squared normed of the $i$-th eigenvector. Note that as already mentioned, 
the incremental strategy requires the transmission of the quantities $\mathbf{r}_{n}$ and $\mathbf{T}_{l o c}^{i}$ from the $i$-th node to the $(i+1)$-th one. The aim of the third iteration is to normalize the updated eigenvalues matrix $\mathbf{U}_{n}$. It is easy to see that the latter can be done by performing sequentially the following steps at the $i$-th node

$$
\mathbf{U}_{n}^{i}=\operatorname{diag}\left(\mathbf{T}_{n}\right)^{1 / 2} \mathbf{U}_{n}^{i}
$$

where $\operatorname{diag}(\cdot)$ denotes the operator that transforms the vector operand to a diagonal matrix. The complete DDPM algorithm is summarized in Table 3-II.

\subsubsection{Cooperative EBSS techniques}

In this subsection the extension of the adaptive EBSS technique to the case of multiple SUs is described. Let us consider that the nodes of SUs network apply the DDPM algorithm of Table 3-II so as to jointly track the subspace of the received data covariance matrix of the aggregate virtual multi-antenna SU system. As it is evident from line 13 of the Table 3-II, each node, at each time index, updates the local estimates of the eigenvalues of the sample covariance matrix. Therefore, each node is also able to compute the test statistics of eqs.(3.4)-(3.6) of the aggregate system and can perform the detection tests independently. Moreover, the test statistics distributions, under the hypothesis $H_{0}$, are again given by Lemma 3.1, by replacing $R_{x}$ with $K * R_{x}$. Observe also that in a dual radio approach, each SU node can employ independently the algorithm of Table 3-I to reach a common decision concerning a detection of a change in the PU activity.

Let us now comment on the specificities of each one of the cooperative EBSS techniques. If the MED method is employed, then it is sufficient to track only the maximum eigenvalue and thus in the DDPM algorithm the cooperating SUs may set $L=1$. Moreover, the distributed orthogonalization steps (lines 15-16 on Table 3-II) are not performed at all, in a way similar to the single SU case. In the case of the MMED method, again in a similar way to the single SU one, each of the SUs tracks

the maximum and the minimum eigenvalues of the covariance matrix by employing 
twice the DDPM for $L=1$. Specifically, first the signal subspace version (with $(+)$ in (3.9)) is employed so as to track the maximum eigenvalue and then the noise subspace version follows (with $(-)$ in $(3.9)$ ) in order to track the minimum one. Recall, that this approach has been adopted in order to avoid the complexity of tracking the complete subspace of the covariance matrix. Of course the steps of each one of the two versions of the DDPM can be applied simultaneously at each time index. Finally, for the adaptive GLRT EBSS method the SU nodes should apply the complete DDPM algorithm with $L=K * R_{x}$.

\subsection{Simulation Results}

In this section, some indicative simulation results are presented in order to evaluate the performance of the adaptive EBSS methods. We assume that the PU transmits a BPSK modulated signal. The step of the FDPM algorithm is set to $\mu=0.8 /\left\|\mathbf{y}_{n}\right\|^{2}$.First, the methods for the single SU case are tested.

In Fig.3-1, the theoretical CDFs of the MED, MMED and GLRT adaptive test statistics under the $\mathcal{H}_{0}$ (Lemma 3.1) are compared to the empirical ones when a block of $N=20$ received signal vectors at the $\mathrm{SU}$ is used to estimate them. The results of 10000 simulations are averaged so as to compute the empirical CDFs for a SU receiver with $R_{x}=4$. As it is shown, the derived theoretical CDFs are very close to the empirical ones even for this small number of received signals.

In Fig.3-2, the performance of the AD-EBSS techniques is compared to the one of the batch techniques in terms of the achieved probability of detection $\mathcal{P}_{d}$ under different SNR values for probability of false alarm $\mathcal{P}_{f}=0.1$. The parameter $a$ of the FDPM algorithm (3.11) is set to $a=0.98$. The performance is examined considering constant channels within each timeslot of duration $N=100$ symbols. The taps of all the involved channels are derived as $\mathcal{C N}(0,1)$ and the results of 10000 realizations are averaged. The decision of the AD-EBSS techniques is taken at the end of each timeslot. That is, we first examine the performance of the AD-EBSS techniques in a batch sense. Note that, even in the batch approach, the AD-EBSS techniques 


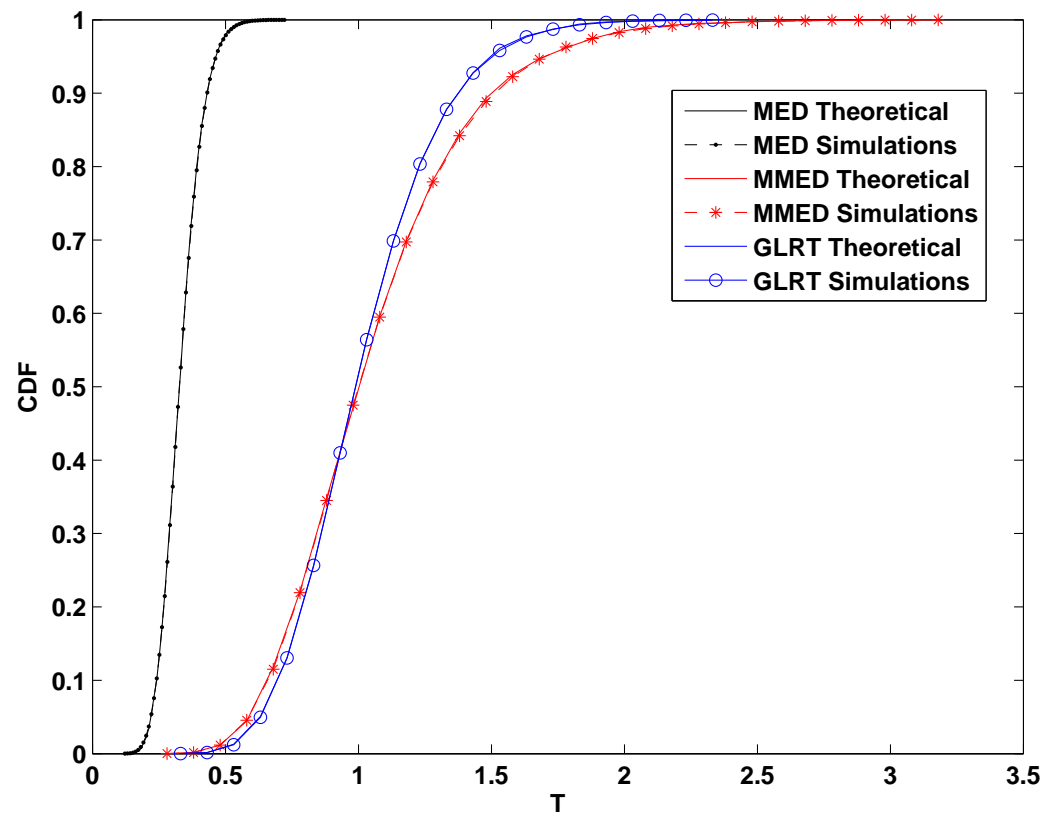

Figure 3-1: CDFs of the test statistics under $\mathcal{H}_{0} \quad$ (Eq.(3.15)-(3.17))

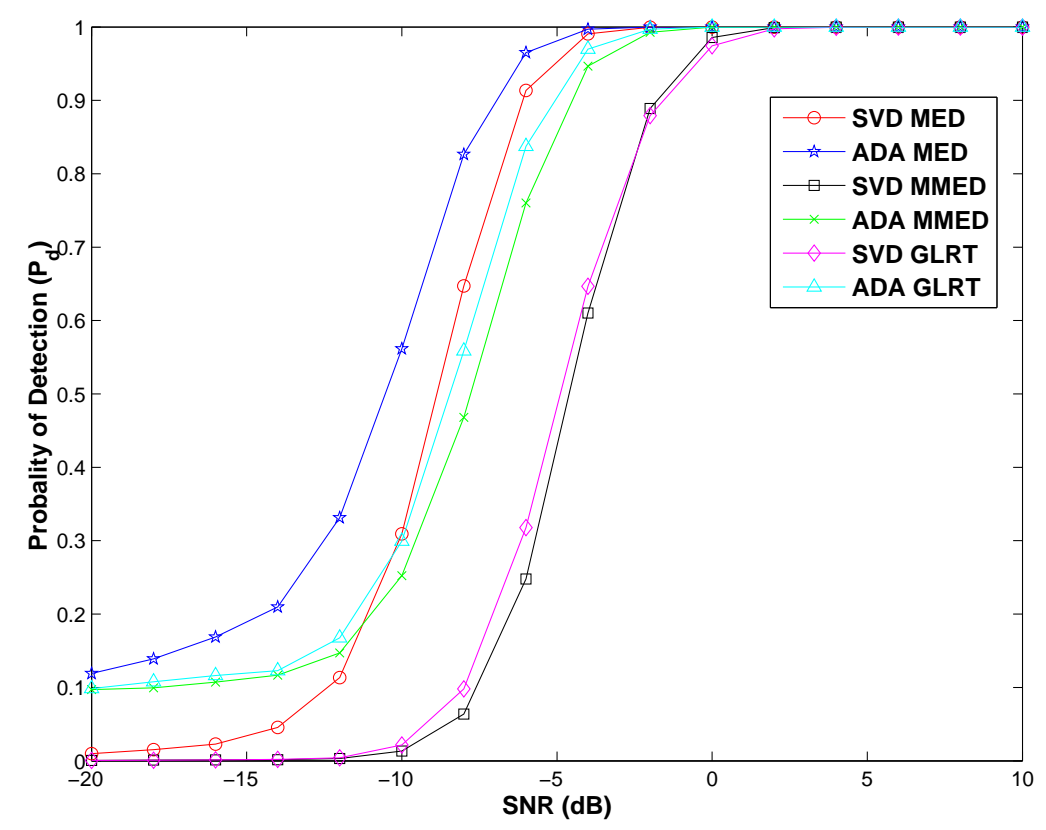

Figure 3-2: Probability of Detection $\left(\mathcal{P}_{d}\right)$ for time-varying channels

exhibit reduced complexity compared to the batch ones, as they do not require the computation of the update of the sample covariance matrix given by Eq.(3.7). As it is shown, the adaptive versions of the test statistics achieve, in general, performance close to the batch ones (or even better, in some cases). This is due to the fact that 


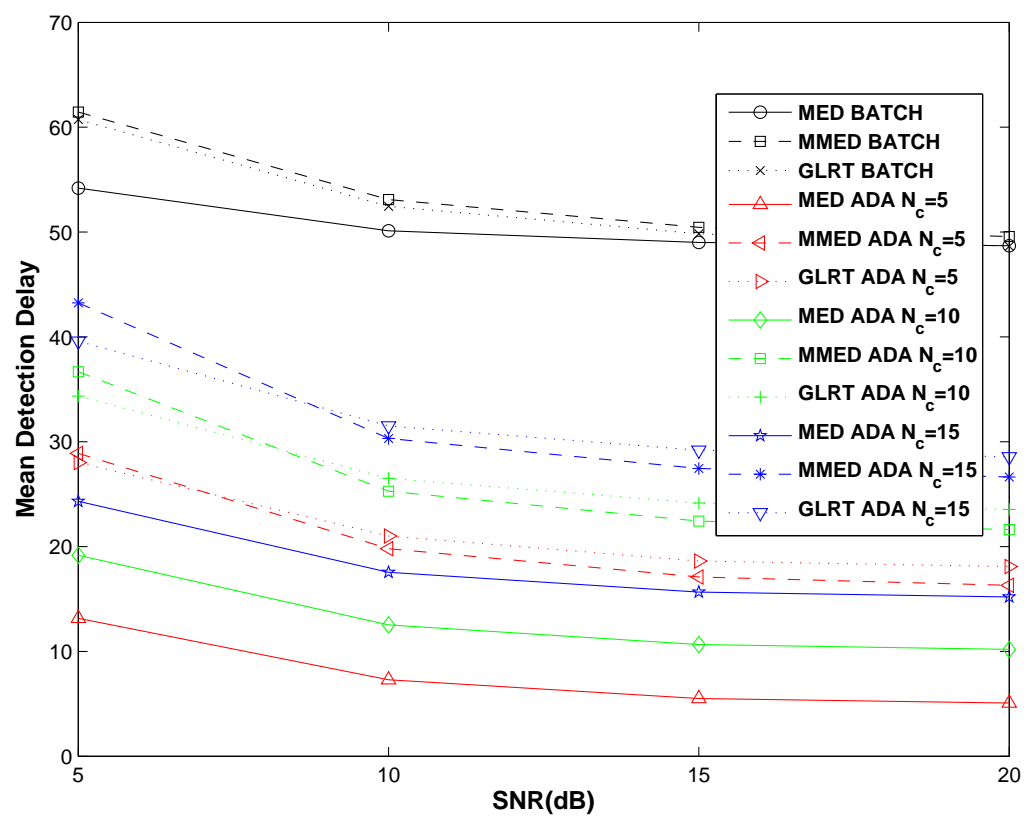

Figure 3-3: Mean Detection Delay of an abrupt change

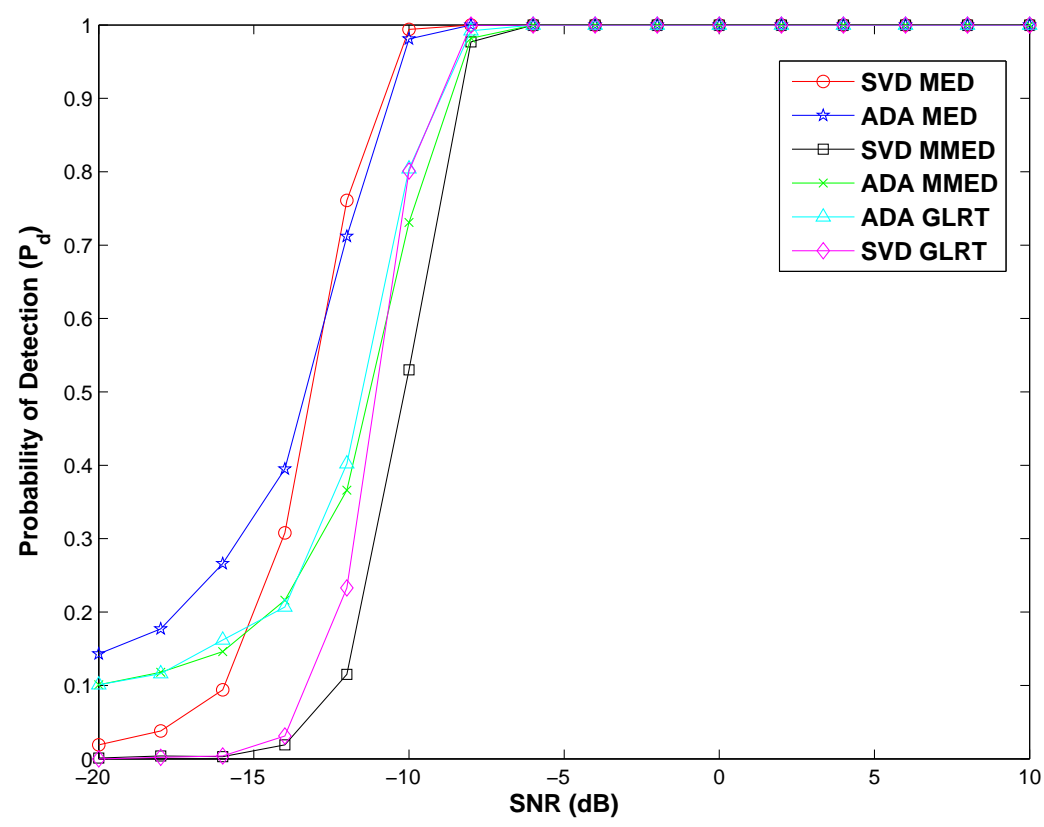

Figure 3-4: Probability of Detection $\left(\mathcal{P}_{d}\right)$ for static channels

the CDFs of the adaptive test statistics Eq.(3.15)-(3.17) are close approximations to the exact ones, whereas for the batch case asymptotic expressions are used.

In Fig.3-3, the same experiment is repeated, though now, time-varying channels are considered. The channels are derived by using a typical Jakes fading simulator 
with $f_{d} T_{s}=0.01$, where $f_{d}$ is the Doppler frequency and $T_{s}$ is the symbol period. Under this fading model, the involved channels are rapidly varying within each sensing period resulting in degradation on the batch detectors performance. In the present simulations, we set $p=0.18$ for a targeted $\mathcal{P}_{f}=0.1$ and $a=0.8$. As it is depicted in Fig.3, the AD-EBSS techniques outperform the batch ones since the former are able to track the variations of the test statistics within the sensing period (see Section $3.3)$.

In Fig.3-4, the mean detection delay in an abrupt change is compared for each one of the batch and the adaptive EBSS techniques for different SNR values in order to evaluate the performance of the method in the dual radio case. We consider timeslots of 1000 symbols in which an abrupt change in the channel state occurred at a random symbol time. The time at which a change occurs is assumed to follow a uniform distribution. The batch EBSS techniques are employed for a block of a 100 symbols. That is, at every block the sample covariance matrix is computed and then, the EVD is applied so as the test statistics to be computed. In the AD-EBSS techniques the algorithm of Table 3-I is used in order to detect a change in the PU activity. The simulations were derived for $N_{c}=5,10$ and 15 respectively, $a=0.98$ and $\mathcal{P}_{f}=0.01$. As it is shown, the adaptive versions detect the change significantly faster than the corresponding batch ones.

Let us now study the multiple SUs case. In Figs.3-5 and 3-6, the experiments of Fig.3-1 for static and Fig.3-2 for time varying channels are repeated, though now 7 SUs of 4 antennas each one are cooperating. In both figures the performance of the cooperative adaptive EBSS methods that employ the DDPM of Table 3-II is compared to the one of the single user adaptive EBSS methods. The performance improvement offered by the cooperative methods is evident and it is due to the fact that they process jointly the samples of the aggregate system of the $K * N U$ receive antennas. In the same figures the performance of a fusion based method, that collects the samples for all the users and performs the batch EBSS techniques, is shown for comparison purposes. As it is shown, the cooperative adaptive EBSS schemes, apart from their benefits due to their completely decentralized nature and their low 


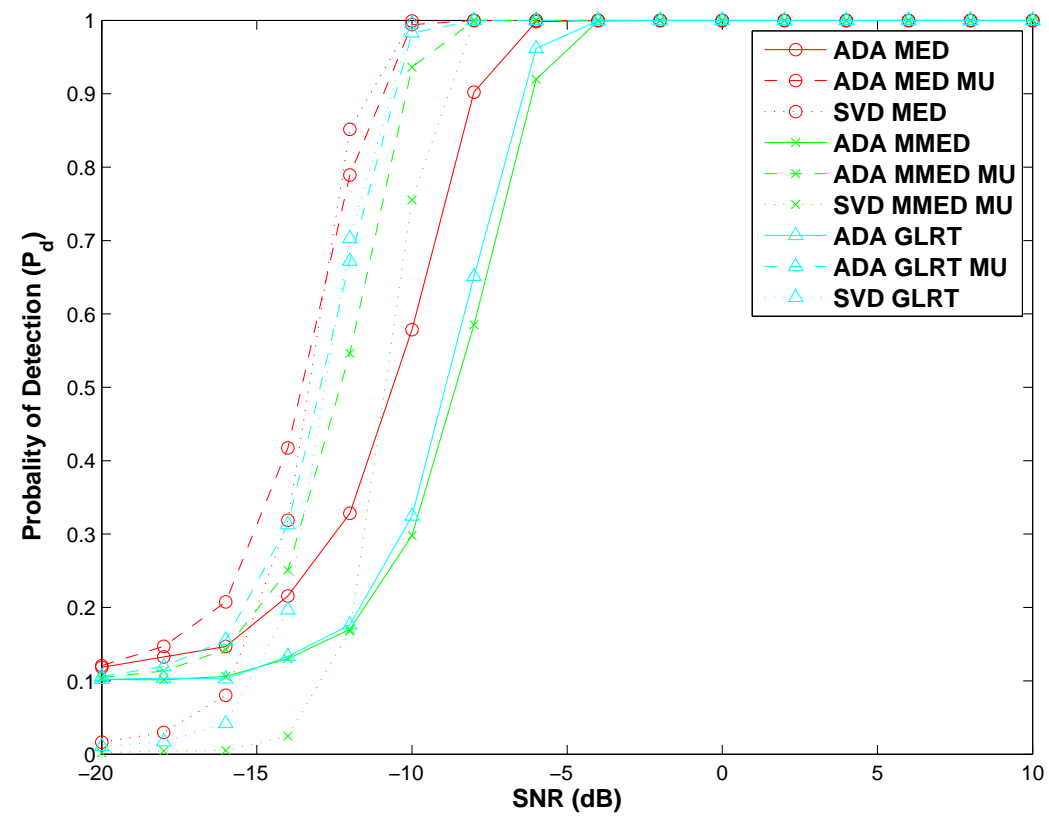

Figure 3-5: Probability of Detection $\left(\mathcal{P}_{d}\right)$ for static channels and $K=7$ SUs

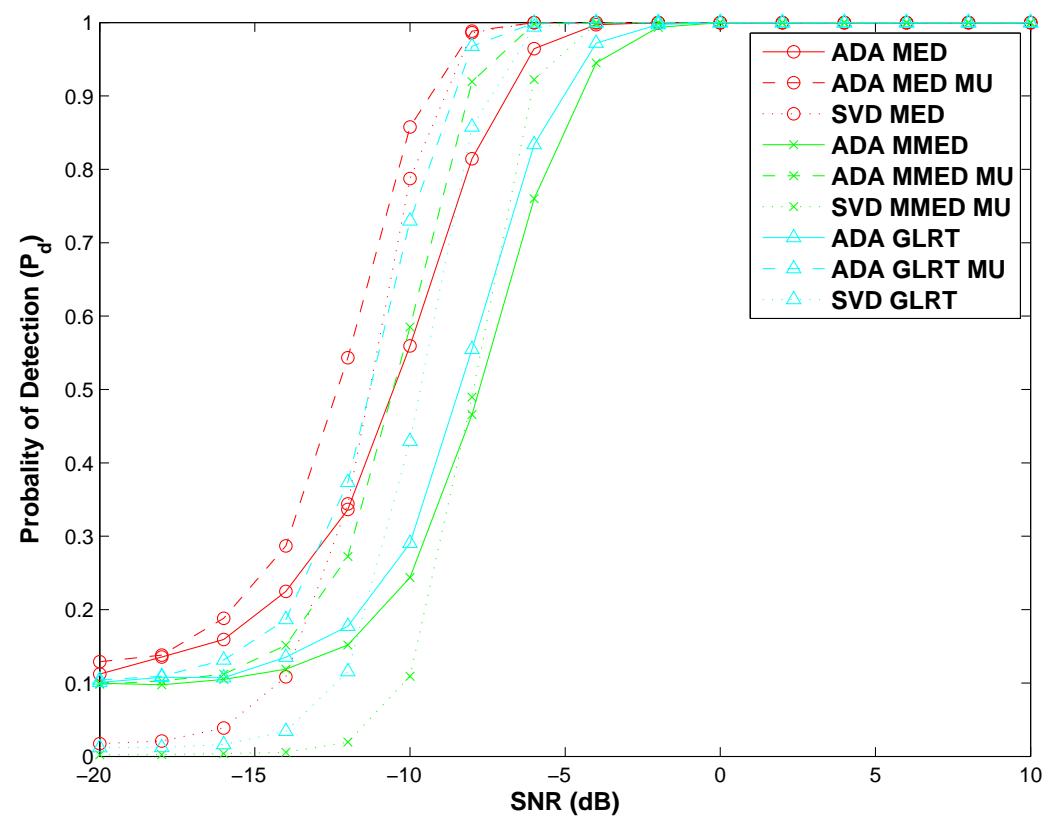

Figure 3-6: Detection Probability $\left(\mathcal{P}_{d}\right)$ for time-varying channels and $K=7$ SUs

complexity, exhibit also improved performance compared to the centralized batch ones at both the static and the time-varying channels, as it was also shown in the single SU case (see Figs.3-1 and 3-2). 


\subsection{Conclusion}

In this chapter, cooperative decentralized adaptive versions of the well-known EBSS techniques were developed for multi-antenna cognitive receivers. The proposed techniques offer low complexity and improved performance especially in cases when time varying channels are involved and/or continuous spectrum monitoring is applied. Moreover due to their decentralized nature the proposed approaches exhibit reduced transmission power consumption during the sensing period and provide robustness against node and link failures. In order to compute the decision thresholds for each one of the adaptive test statistics, close approximations for the associated distribution functions were derived. A novel distributed subspace tracking method was also derived as a constituent part of the proposed decentralized EBSS techniques. The new distsributed ST method enable the SUs to track jointly the subspace of their received signals in a completely decentralized manner. The performance of the proposed EBSS techniques was verified via indicative simulations and compared to those of the corresponding batch centralized approaches. 


\subsection{Appendix}

\section{A Derivation of the Distribution of the MMED Test Statis- tic under the $\mathcal{H}_{0}$ Hypothesis}

Let us assume that two RVs $T_{1}$ and $T_{2}$ follow the same distribution with that of the MED test statistic. The CDF of the later distribution is given by eq.(3.15). The corresponding Probability Density Function (PDF) is computed by taking the derivative of eq.(3.15). That is,

$$
f_{T_{i}}\left(\tau_{i}\right)=\frac{\xi^{\rho}}{\Gamma(\rho)} \tau_{i}^{\rho-1} e^{-\rho \tau_{i}}
$$

We are interested in the distribution of the random variable $Y_{1}=T_{1} / T_{2}$. Let us also define the auxiliary random variable $Y_{2}=T_{2}$. Thus we have,

$$
\begin{aligned}
& f_{1}\left(t_{1}, t_{2}\right)=\frac{t_{1}}{t_{2}} \\
& f_{2}\left(t_{1}, t_{2}\right)=t_{2},
\end{aligned}
$$

where $\tau_{i} \in \mathbb{R}^{+}$. The inverse functions of the ones of eq.(3.31) are given by

$$
\begin{aligned}
& f_{1}^{-1}\left(t_{1}, t_{2}\right)=t_{1} \tau_{2} \\
& f_{2}^{-1}\left(t_{1}, t_{2}\right)=t_{2},
\end{aligned}
$$

The joint PDF of variables $Y_{1}$ and $Y_{2}$ is given by

$$
f_{Y_{1}, Y_{2}}\left(y_{1}, y_{2}\right)=f_{T_{1}, T_{2}}\left(f_{1}^{-1}\left(y_{1}, y_{2}\right), f_{2}^{-1}\left(y_{1}, y_{2}\right)\right)\left|J\left(y_{1}, y_{2}\right)\right|,
$$

where $J\left(y_{1}, y_{2}\right)=\frac{\partial\left(t_{1}, t_{2}\right)}{\partial\left(y_{1}, y_{2}\right)}$ is the Jacobian matrix of the transformation and $\left|J\left(y_{1}, y_{2}\right)\right|=$ $y_{2}$ is its determinant.

Observe now that, since the eigenvalues are estimated via eq.(3.11), we can assume statistical independence between them. That is, the joint PDF of the variables under consideration $T_{1}$ and $T_{2}$ can be computed as the product of the corresponding marginal 
ones (eq.(3.15)). Therefore, from Eq.(3.33) the joint PDF of $Y_{1}$ and $Y_{2}$ is given by

$$
\begin{array}{r}
f_{Y_{1}, Y_{2}}\left(y_{1}, y_{2}\right)=f_{T_{1}}\left(y_{1} y_{2}\right) f_{T_{2}}\left(y_{2}\right) y_{2}= \\
\frac{\xi^{2 \rho}}{\Gamma^{2}(\rho)} y_{1}^{\rho-1} y_{2}^{2 \rho-1} e^{-\xi y_{2}\left(y_{1}+1\right)}
\end{array}
$$

In order to compute the marginal PDF of RV $Y_{1}$, we integrate the joint one of eq.(3.35) with respect to $y_{2}$. That is

$$
\begin{aligned}
f_{Y_{1}}\left(y_{1}\right) & =\frac{y_{1}^{\rho-1}}{\xi^{2 \rho} \Gamma^{2}(\rho)} \int_{0}^{+\infty} y_{2}^{2 \rho-1} e^{-\xi y_{2}\left(y_{1}+1\right)} d y_{2} \\
& =\frac{y_{1}^{\rho-1} \xi^{2 \rho} \Gamma(2 \rho)}{\left(1+y_{1}\right)^{2 \rho} \xi^{2 \rho} \Gamma^{2}(\rho)} \\
& =\frac{y_{1}^{\rho-1}}{B(\rho, \rho)\left(1+y_{1}\right)^{2 \rho}}
\end{aligned}
$$

where the following property of the beta function [106] was used

$$
B\left(\rho_{1}, \rho_{2}\right)=\int_{0}^{1} x^{\rho_{1}}(1-x)^{\rho_{2}-1} d x=\frac{\Gamma\left(\rho_{1}+\rho_{2}\right)}{\Gamma\left(\rho_{1}\right) \Gamma\left(\rho_{2}\right)} .
$$

By integrating eq.(3.36) we derive the corresponding CDF of the beta prime distribution given by Eq.(3.17) of Lemma 1 and the proof is completed. 


\section{Chapter 4}

\section{A Cooperative Uplink Transmission Technique with Improved Diversity-Multiplexing Tradeoff}

\subsection{Introduction}

In Section 1.5 the concept of cooperative systems was described as an alternative way to achieve spatial diversity via virtual antenna arrays formed by single antenna nodes. As it was mentioned, the cooperative communications field was extensively researched the past years and a number of different papers were cited.

The majority of the aforementioned papers aim to develop techniques that provide cooperative diversity in half-duplex nodes at the expense of multiplexing gain. In fact, the transmissions exhibit severe multiplexing gain loss since a transmission from the source to the destination node requires two orthogonal channels (source to relay(s) and relays(s) to destination) that in general are created via a typical time division approach [30].

To that end a number of different approaches have already been suggested in 
order to improve the multiplexing gain of the resulting cooperative techniques. In the first one [107]-[109], cooperative schemes are proposed in which the source to relay(s) and the relays(s) to destination channels are non-orthogonal. The resulting techniques exhibit a better DMT than the initial cooperative schemes [30] at the expense of noise interference caused by the simultaneous transmissions of the nodes. In [110]-[111] a superposition coding approach was proposed where the multiplexing gain is improved by permitting each user to transmit a linear combination of its own information and other users' information. At the destination node, the superposition decoder extracts multiple modulated symbols by using log-likelihood ratios which requires precise knowledge of the instantaneous fading gains, the noise variance and the linear combination coefficients that the superposition codeword employs. Therefore, an improper knowledge of the combination coefficients leads to severe performance degradation. Moreover, a method that provides and efficient design for the latter coefficients in in general unknown.

In typical wireless systems (i.e. cellular networks), the mobile users are communicating via base stations that are deployed in specific areas. The base stations can in general be complex systems based on large multi-antenna arrays, whereas the mobile users' terminals are based on simple single antenna structures having also power restrictions. Therefore, cooperative protocols can be employed so as to improve the quality / reduce the required power of the uplink transmissions by establishing cooperation among single-antenna users that are in close proximity to each other. The motivation behind the present work is to develop uplink techniques for half duplex systems that apply single-antenna user cooperation in order to create a virtual MIMO system along with the multi-antenna base-station. The proposed techniques aim to provide spatial diversity in order to improve the quality of the uplink transmissions and also improved multiplexing gains.

The contributions presented in this chapter are the following ones.

At first, we develop and study the performance of the uplink cooperative transmission technique suggested in [74]-[76] for DF cooperative systems. The proposed technique exploits properly the structure of the virtual MIMO channel matrix [82] to 
create multiple independent channels (singular channels) through which the symbols are transmitted simultaneously to the destination. Theoretical results are derived for the outage probability and the DMT [112] of the proposed scheme. As it is shown the proposed technique achieves greater diversity than a SIMO system where each user transmits without cooperation its data to the multi-antenna destination and greater multiplexing gain than the conventional DF protocol of [30]. In fact, it is noteworthy that the system is able to choose among different options concerning the achieved diversity and multiplexing gain by properly configuring its parameters. That is the proposed technique is one of the most flexible ones since the majority of the existing techniques present fixed diversity and multiplexing gains. The theoretical results are verified by typical simulations in which the performance of the proposed scheme is evaluated for different values of the involved parameters and compared to the one of a non cooperative SIMO system.

The rest of the chapter is organized as follows. In Section 4.2, the proposed technique is described. In Section 4.3 we present the corresponding theoretical performance analysis. In Section 4.4, representative simulation results are shown and discussed, and finally, Section 4.5 concludes the chapter.

\subsection{System's Description}

In this section the system description is given. Let us consider a system with a singleantenna source and a $M$ antenna destination, and let us also assume that $N$ relays are available to participate in a cooperative transmission scheme. The relays are assumed to be other adjacent users that help the scheduled one to transmit its data to the destination via employing a cooperative transmission protocol. The idea is based on the fact, that each user can find other users in its close proximity that have strong channels and form a virtual MIMO system with the multiple antennas of the base station (destination) in order to improve the quality of the uplink transmission.

The "matching" of a user with its partners can be done by employing opportunistic strategies, i.e. relay selection schemes [113], though any further discussion is beyond 


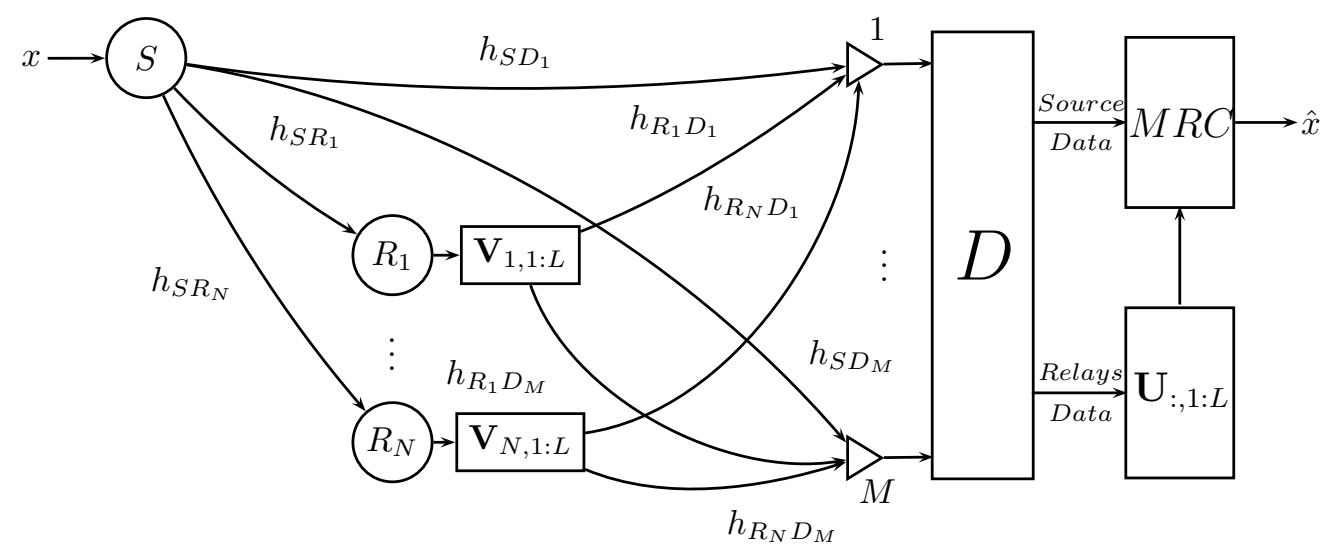

Figure 4-1: The proposed system.

$$
\begin{array}{|c|c|}
\hline S \rightarrow R_{1 \ldots N}, S \rightarrow D & R_{1 \ldots N} \rightarrow D \\
\hline T_{1} & T_{2} \\
\hline
\end{array}
$$

Table 4-I: Medium access control.

the scopes of the present paper.

Let us now proceed to the system description. A system with $N$ relays and $M$ antennas at the receiver is depicted in Figure 4-1. The time frame is divided into two periods (see Table 4.I). During time period $T_{1}$ the source transmits $L$ symbols $x(n), 1 \leq n \leq L \in[1, \min \{M, N\}]$, in $L$ consecutive time slots, to the $N$ relays and to the destination. For the $n-t h$ symbol the samples received at the destination and the relay nodes are given by

$$
\begin{gathered}
\mathbf{y}_{D}(n)=\sqrt{\frac{P_{S}}{2}} \mathbf{h}_{S D} x(n)+\mathbf{w}(n) \\
y_{R_{i}}(n)=\sqrt{\frac{P_{S}}{2}} h_{S R_{i}} x(n)+w_{i}(n), \quad 1 \leq i \leq N,
\end{gathered}
$$

where $\mathbf{y}_{D}(n)=\left[y_{D_{1}}(n) \ldots y_{D_{M}}(n)\right]^{T}$ is the received vector at the destination, $\mathbf{h}_{S D}=\left[\begin{array}{lll}h_{S D_{1}} & \ldots & h_{S D_{M}}\end{array}\right]^{T}$ is a vector containing the taps of the flat fading channels between the source and the destination antennas, $h_{S R_{i}}$ is the tap of the flat fading channel between the source and the $i-t h$ relay, $x(n)$ is the $n-t h$ transmitted symbol, 
$\mathbf{w}(n) \in \mathcal{C}^{M \times 1}$ and $w_{i}(n) \in \mathcal{C}$ are variables of complex additive noise of variance $\sigma_{w}^{2}$ modelled as $\mathcal{C N}\left(0, \sigma_{w}^{2}\right)$ and $P_{S}$ is the power constraint of the source node. It is assumed that the involved channels undergo Rayleigh block fading with block size equal to $L+1$ time symbols. The corresponding flat fading coefficients capture the effects of path-loss and shadowing and are modelled as $\mathcal{C N}\left(0, \gamma_{S R}^{2}\right), \mathcal{C N}\left(0, \gamma_{S D}^{2}\right)$ and $\mathcal{C N}\left(0, \gamma_{R_{i} D}^{2}\right), 1 \leq i \leq N$ for the source-to-relays, source-to-destination and the relaysto-destination channels respectively. As it was described, we assume in general that the relays are other adjacent users that help the one that plays the role of the source to transmit its information to the base station (destination). Therefore, from now and on, we assume that $\mathcal{C N}\left(0, \gamma_{R_{i} D}^{2}\right)=\mathcal{C N}\left(0, \gamma_{S D}^{2}\right)$ for simplicity. The relay nodes detect the symbols based on the received signals described by (4.2), and subsequently, at the $L+1$ time slot (period $T_{2}$ in Figure 1.2), the relays that have correctly detected all the $L$ symbols forward them simultaneously to the destination. It is evident that, the number of the relays that correctly decoded all the symbols must be at least $L$, that is $N-K \geq L$. In case that $N-K<L$, we assume for simplicity, that the relays do not forward any symbol at all and only the direct source-destination transmission path is used.

Note that the assumption that a relay knows if a symbol is correctly detected in the DF protocol case, is typical in the relevant literature [30]. Of course as it is also evident, the relays should acknowledge the other ones and the destination if they will participate in the transmission period $T_{2}$. Similar assumptions are typical also in the literature of distributed space-time coding for cooperative networks where the problem is tackled by assuming 1-bit acknowledgement broadcasts from each relay. For example, if a relay detects an error in one of the $L$ received symbols sets the bit to one state in order to acknowledge the other ones that it will not be able to participate during transmission phase $T_{2}$ (see [114]) and references within).

Let us now assume that $N-K \geq L$, so that each relay is able to forward a superposition of the $L$ symbols (Fig. 1). The $i-t$ th relay's superposition weights are the first $L$ elements of the $i-t h$ row of the right singular vectors' matrix $\mathbf{V}_{1: N, 1: L}$. 
The latter is computed by the SVD of the virtual MIMO channel matrix

$$
\mathbf{H}_{R D}=\left[\begin{array}{ccc}
h_{R_{1} D_{1}} & \cdots & h_{R_{N} D_{1}} \\
\vdots & \ddots & \vdots \\
h_{R_{1} D_{M}} & \cdots & h_{R_{N} D_{M}}
\end{array}\right]
$$

where $h_{R_{i} D_{j}} \sim \mathcal{C N}\left(0, \gamma_{R D}^{2}\right)$ is the tap of the channel between the $i-t h$ relay and the $j-$ th destination antenna. Thus, during the transmission period $T_{2}$ the received samples at the destination node are

$$
\mathbf{y}_{D}(L+1)=\sqrt{\frac{P_{S}}{2}} \mathbf{H}_{R D} \mathbf{x}^{\prime}(L+1)+\mathbf{w}(L+1)
$$

where $\mathbf{x}^{\prime}(L+1)=\mathbf{V}_{1: N, 1: L} \mathbf{x}(L+1), \mathbf{H}_{R D}=\mathbf{U} \mathbf{\Sigma} \mathbf{V}^{H}, \mathbf{x}(L+1)=[\hat{x}(1), \cdots, \hat{x}(L)]^{T}$ is a vector with the $L$ symbols, and $\mathbf{w}(L+1) \in \mathcal{C}^{M \times 1}$. The received vector at the destination is post-coded with the corresponding left singular vector sub-matrix $\mathbf{U}_{1: M, 1: L}$, resulting in

$$
\mathbf{y}_{D}^{\prime}=\mathbf{U}_{1: M, 1: L}^{H} \mathbf{y}_{D}(L+1)=\sqrt{\frac{P_{S}}{2}} \mathbf{\Sigma} \mathbf{x}(L+1)+\mathbf{w}^{\prime}(L+1),
$$

where $\boldsymbol{\Sigma}$ is the singular values' diagonal matrix and $\mathbf{w}^{\prime}(L+1) \in \mathcal{C}^{L \times 1}$ due to the singular vectors' unitary property. Thus, we have an equivalent system in which the $L$ symbols are transmitted via the respective independent parallel singular channels employing equal power allocation strategy. Of-course the performance of the proposed technique can be further improved by employing a water-filing based power allocation approach at the relays-to destination transmission [82], though for simplicity we consider here the equal power allocation strategy only. Now, note that the $i-t h$ channel tap is equal to the $i-t h$ singular value. Since the singular values are independent of the taps $h_{S D_{i}}$ we can use a combination of (4.1) and (4.5) to extract higher diversity gain. More precisely, for each symbol that was correctly detected from the relays we have $M$ transmission paths from (4.1) and one path from (4.5). The latter path is the corresponding singular channel which contributes implicitly to 
the overall diversity gain. Thus, Maximum Ratio Combining (MRC) can be applied on the equivalent SIMO system, i.e.,

$$
\mathbf{y}_{D}^{i}=\mathbf{h}_{e q}^{i} \mathbf{x}_{i}+\mathbf{w}_{i}, \quad 1 \leq i \leq L
$$

where $\mathbf{y}_{D}^{i}=\left[\mathbf{y}_{D}^{T}(i), y_{D}^{\prime}{ }^{i}(L+1)\right]^{T}, y_{D}^{\prime i}(L+1)$ is the $i-t h$ element of $\mathbf{y}_{D}^{\prime}, \sigma_{i}$ is the $i-$ th singular value, $\mathbf{h}_{e q}^{i}=\sqrt{\frac{P_{S}}{2}}\left[\mathbf{h}_{S D}^{T}, \sigma_{i}\right]^{T}, \mathbf{w}_{i}=\left[\mathbf{w}^{T}(i), w_{i}^{\prime}(L+1)\right]^{T}$ and $w_{i}^{\prime}(L+1)$ is the $i-t h$ element of $\mathbf{w}^{\prime}(L+1)$.

\subsection{Performance Analysis}

In this section theoretical results concerning the performance of the proposed scheme are derived. At first the outage probability of the technique is derived. Then the corresponding DMT curve is computed.

\subsubsection{Outage Probability Analysis}

The following theorem provides the theoretical outage probability of the technique under Rayleigh fading.

Theorem 4.4 The outage probability of the proposed technique for a system with $M$ destination antennas, $N_{r}$ relays, block size $L$ and targeted data rate $R$ bits/sec under Rayleigh fading conditions is given by

$$
\begin{aligned}
P_{\text {out }}= & \sum_{K=0}^{N-L}\left(\begin{array}{l}
N \\
K
\end{array}\right)\left(1-e^{-\frac{{ }^{2} R-1}{0.5 \gamma_{S R}^{2} \xi}}\right)^{K} e^{-\frac{\left(2^{R}-1\right)(N-K)}{0.5 \gamma_{S R}^{2} \xi}} \times \\
& \left.\left(\frac{1}{0.5 \gamma_{S D}^{2}}\right)^{M}\left(\frac{1}{0.5 \gamma_{S D}^{2} \bar{\lambda}_{L}}\right)^{\beta(K)} G_{\beta(K)+M+1, \beta(K)+M+1}^{\beta(K)+M+1,0}\left(\begin{array}{c}
\boldsymbol{\Psi}_{1} \\
\boldsymbol{\Psi}_{2}
\end{array}\right), e^{-\frac{2^{R}-1}{\xi}}\right)+ \\
& \sum_{K=N-L+1}^{N}\left(\begin{array}{l}
N \\
K
\end{array}\right)\left(1-e^{-\frac{2^{R}-1}{0.5 \gamma_{S R}^{2} \xi}}\right)^{K} e^{-\frac{\left(2^{R}-1\right)(N-K)}{0.5 \gamma_{S R}^{2} \xi}}\left(1-e^{-\frac{2^{R}-1}{0.5 \gamma_{S D}^{2} \xi}} \sum_{m=0}^{M-1} \frac{\left(\frac{2^{R}-1}{0.5 \gamma_{S D}^{2} \xi}\right)^{m}}{m !}\right)
\end{aligned}
$$


where $\xi=\frac{P_{S}}{\sigma_{w}^{2}}, \bar{\lambda}_{L}$ is the mean of the squared eigenvalue of matrix $\mathbf{H}_{R D}$ when its entries are distributed as $\mathcal{C} N(0,1), \beta(K)=(N-K-L+1)(M-L+1), G_{p, q}^{m, n}$ is the so-called MeijerG function [87] having the vector operands $\boldsymbol{\Psi}_{1}$ and $\boldsymbol{\Psi}_{2}$ defined as

$$
\begin{aligned}
& \boldsymbol{\Psi}_{1}=[\underbrace{\left(1+\frac{1}{0.5 \gamma_{S D}^{2}}\right), \ldots,\left(1+\frac{1}{0.5 \gamma_{S D}^{2}}\right)}_{M-\text { terms }}, \underbrace{\left(1+\frac{1}{0.5 \gamma_{S D}^{2} \bar{\lambda}_{L}}\right), \ldots,\left(1+\frac{1}{0.5 \gamma_{S D}^{2} \bar{\lambda}_{L}}\right)}_{\beta(K)-\text { terms }}, 1] \\
& \boldsymbol{\Psi}_{2}=[\underbrace{\left(\frac{1}{0.5 \gamma_{S D}^{2}}\right), \ldots,\left(\frac{1}{0.5 \gamma_{S D}^{2}}\right)}_{M-\text { terms }}, \underbrace{\left(\frac{1}{0.5 \gamma_{S D}^{2} \bar{\lambda}_{L}}\right), \ldots,\left(\frac{1}{0.5 \gamma_{S D}^{2} \bar{\lambda}_{L}}\right)}_{\beta(K)-\text { terms }}, 0]
\end{aligned}
$$

respectively.

Proof Let us first provide some notations. The outage probability of an $1 \times M M I M O$ system for a given rate $R$ is defined as

$$
P_{\text {out }}(R)=P\left\{\log \left(1+\|\mathbf{h}\|^{2} \xi\right)<R\right\}=F_{\eta}\left(2^{R}-1\right)
$$

where $F_{\eta}(\cdot)$ is the Cumulative Distribution Function (CDF) of the Random Variable $(R V) \eta=\|\mathbf{h}\|^{2} . P_{(K)}$ and $\overline{P_{(K)}}$ denote the probability that an outage event occurs and does not occur in $K$ relays, respectively, $P_{o u t}^{e q(N-K)}$ is the outage probability of the overall equivalent system of eq. (4.6) when $N-K \geq L$ relays do not exhibit an outage event, $P_{\text {out }}^{S I M O}$ denotes the outage probability of the generic SIMO system consisted only of the direct paths between the source and the destination antennas when $N-K<L$, and $P_{\text {out }}^{S I S O}$ is the outage probability of a SISO system (as the one between the source and a relay). We claim that the outage probability of the proposed technique can be expressed as

$$
P_{\text {out }}=\sum_{K=0}^{N-L}\left(\begin{array}{l}
N \\
K
\end{array}\right) P_{(K)}\left(\overline{P_{(N-K)}}\right) P_{\text {out }}^{e q(N-K)}+\sum_{K=N-L+1}^{N}\left(\begin{array}{l}
N \\
K
\end{array}\right) P_{(K)}\left(\overline{P_{(N-K)}}\right) P_{\text {out }}^{S I M O}
$$

Observe that equation (4.10) consists of two sums that correspond to the two distinct 
cases that appear when the technique is applied. As it was described in Section II, the relays forward the data during the $T_{2}$ phase if the number of the relays that correctly decoded the source data are $N-K \geq L$. In this case the equivalent system of eq.(6) is used for symbol detection at the destination. Therefore, one can compute the outage probability of the system by weighting the outage probability of the last system by the probability that exact $K$ relays exhibit an outage event, given by $\left(\begin{array}{l}N \\ K\end{array}\right) P_{(K)}\left(\overline{P_{(N-K)}}\right)$, where the binomial term $\left(\begin{array}{l}N \\ K\end{array}\right)$ is used to count for all the possible combinations of $K$ relays, and taking the summation for $0 \leq K \leq N-L$. The latter steps result in the first term of the eq.(4.10). By following the same procedure one can derive also the second sum of eq.(4.10) that corresponds to the case $N-K<L$ and so the remaining relays number is not sufficient in order to forward the data to the destination and therefore remain silent during the transmission period $T_{2}$. That $i s$, the destination relies only on the transmission of period $T_{1}$ for the symbol detection and the outage probability of the system equals to the one of a SIMO one having $M$ receiver antennas.

Let us now proceed further with the proof. As it is evident both of the sum terms of eq. (4.10) include the term $\left(\begin{array}{c}N \\ K\end{array}\right) P_{(K)}\left(\overline{P_{(N-K)}}\right)$ which as it was already stated equals the probability that exactly $K$ out of the $N$ relays exhibit an outage event during the source-to-relays transmission phase $T_{1}$. If we assume that the relays have independent fading channel coefficients the outage event at exactly $K$ relays can be expressed as

$$
\begin{aligned}
\left(\begin{array}{l}
N \\
K
\end{array}\right) P_{(K)}\left(\overline{P_{(N-K)}}\right) & =\left(\begin{array}{l}
N \\
K
\end{array}\right)\left(P_{\text {out }}^{S I S O}\right)^{K}\left(1-P_{\text {out }}^{S I S O}\right)^{N-K} \\
& =\left(\begin{array}{l}
N \\
K
\end{array}\right)\left(1-e^{-\frac{2^{R}-1}{0.5 \gamma_{S}^{2} \xi}}\right)^{K} e^{-\frac{\left(2^{R}-1\right)(N-K)}{0.5 \gamma_{S R}^{2} \xi}},
\end{aligned}
$$

where we have used the formula $P_{\text {out }}^{S I S O}=1-e^{-\frac{2^{R}-1}{0.5 \gamma_{S R}^{2} \xi}}$ [115] that gives the outage probability of a SISO system having the same fading parameters and transmission power as the ones considered in the source-to-relays transmission period $T_{1}$.

Let us, now consider the case $N-K<L$ As it was already described, the outage probability of the system equals to the one of a SIMO one having $M$ receiver antennas. For the given fading conditions and source power transmission, the latter probability 
according to [115] is given by

$$
P_{\text {out }}^{S I M O}=1-e^{-\frac{{ }_{2}^{R}-1}{0.5 \gamma_{S D}^{2} \xi}} \sum_{m=0}^{M-1} \frac{\left(\frac{2^{R}-1}{0.5 \gamma_{S D}^{2} \xi}\right)^{m}}{m !}
$$

In order to complete the proof, we finally need to derive the outage probability of the equivalent system of eq.(6) $P_{\text {out }}^{\text {eq(N-K) }}$ when $N-K \geq L$. Since in the destination a $M R C$ scheme is applied on the equivalent parallel independent channels $\mathbf{h}_{\text {eq }}^{i}, 1 \leq i \leq L$, the received $S N R$ can be expressed as $\left\|\mathbf{h}_{\text {eq }}^{i}\right\|^{2}, 1 \leq i \leq L$. Now according to system description we have that

$$
\left\|\mathbf{h}_{e q}^{i}\right\|^{2}=0.5\left(\left|h_{S D_{1}}\right|^{2}+\cdots+\left|h_{S D_{M}}\right|^{2}\right)+0.5 \sigma_{i}^{2}=0.5\left\|\mathbf{h}_{S D}\right\|^{2}+0.5 \sigma_{i}^{2}, 1 \leq i \leq L
$$

Eq.(4.13) is a sum of positive terms and thus one can see that $\left\|\mathbf{h}_{e q}^{L}\right\|^{2} \leq\left\|\mathbf{h}_{e q}^{L-1}\right\|^{2} \leq$ $\cdots \leq\left\|\mathbf{h}_{\text {eq }}^{1}\right\|^{2}$ due to the fact that $0 \leq \sigma_{L} \leq \sigma_{L-1} \leq \cdots \leq \sigma_{1}$. The following observation leads to the conclusion that the outage probability of the equivalent system equals the outage probability of its weakest in terms of the received SNR channel, that is the one involving the $L-t h$ eigenvalue. Thus, in order to compute the required outage probability the distribution of the scalar $\left\|\mathbf{h}_{\text {eq }}^{L}\right\|^{2}$ Random Variable (RV) must be derived. Now observe the second equation of eq.(4.13). The $R V \chi=0.5\left\|\mathbf{h}_{S D}\right\|^{2}$ is a scaled sum of squared $R V$ s distributed as $\mathcal{C N}\left(0, \gamma_{S D}^{2}\right)$ and therefore has CDF given by

$$
F_{\chi}(x)=1-e^{-\frac{x}{0.5 \gamma_{S D}^{2}}} \sum_{m=0}^{M-1} \frac{\left(\frac{x}{0.5 \gamma_{S D}^{2}}\right)^{m}}{m !}
$$

Note that, the outage of SIMO system given at eq.(4.12) is derived by evaluating the CDF of eq.(4.14) at point $\frac{2^{R}-1}{\xi}$. Moreover, for the $R V \psi=0.5 \sigma_{i}^{2}$ we can use the results of [88] that concern the marginal distribution of the $L-$ th squared eigenvalue of a MIMO channel under Rayleigh fading, $\mathcal{C N}\left(0, \gamma_{S D}^{2}\right)$, and derive the corresponding 
CDF which is given by

$$
F_{\psi}(y)=1-e^{-\frac{y}{0.5 \gamma_{S D}^{2}}} \sum_{m=0}^{\beta(K)-1} \frac{\left(\frac{y}{0.5 \gamma_{S D}^{2}}\right)^{m}}{m !}
$$

From eqs.(4.14)-(4.15), it is evident that the $R V \zeta=\left\|\mathbf{h}_{\text {eq }}^{L}\right\|^{2}$ is the sum of the independent $R V s \chi$ and $\psi$ which follow the gamma distribution with different parameters each one. By using the results of [116] we can show that the CDF of the $R V \zeta$ is given by

$$
F_{\zeta}(z)=\left(\frac{1}{0.5 \gamma_{S D}^{2}}\right)^{M}\left(\frac{1}{0.5 \gamma_{S D}^{2} \bar{\lambda}_{L}}\right)^{\beta(K)} G_{\beta(K)+M+1, \beta(K)+M+1}^{\beta(K)+M+1,0}\left(\begin{array}{c}
\boldsymbol{\Psi}_{1} \\
\boldsymbol{\Psi}_{2}
\end{array} \mid, e^{-z}\right)
$$

where the vector operands $\boldsymbol{\Psi}_{1}$ and $\boldsymbol{\Psi}_{2}$ were defined at eq.(4.8). Finally, by evaluating the CDF of eq.(4.16) at $\frac{2^{R}-1}{\xi}$ we get the required outage probability of the equivalent system given by

$$
P_{\text {out }}^{e q(N-K)}=\left(\frac{1}{0.5 \gamma_{S D}^{2}}\right)^{M}\left(\frac{1}{0.5 \gamma_{S D}^{2} \bar{\lambda}_{L}}\right)^{\beta(K)} G_{\beta(K)+M+1, \beta(K)+M+1}^{\beta(K)+M+1,0}\left(\begin{array}{c}
\boldsymbol{\Psi}_{1} \\
\boldsymbol{\Psi}_{2}
\end{array} \mid, e^{-\frac{{ }_{2}^{R}-1}{\xi}}\right)
$$

Now by plugging in the results of eqs.(4.11), (4.12) and (4.17) into eq.(4.10) we get eq.(4.7) which completes the proof.

\subsubsection{Diversity-Multiplexing Tradeoff Analysis}

Let us now proceed to the Derivation of the DMT curve of the proposed scheme. According to [112], the diversity gain for a target rate $R=r \log _{2}(\xi)$ where we remind $\xi=\frac{P_{S}}{\sigma_{w}^{2}}$ is the transmission SNR, is defined as

$$
d(r)=-\lim _{\xi \rightarrow \infty} \frac{\log _{2}\left(P_{e}(\xi)\right)}{\log _{2}(\xi)}
$$

where $P_{e}(\xi)$ is the error probability for the given SNR and $r$ is the multiplexing gain. In [112] the authors used the fact that a communication can be always error free by 
setting the rate lower than the capacity of the channel so as to avoid an outage event to lower bound the error probability by the outage one. That is,

$$
\lim _{\xi \rightarrow \infty} \frac{\log _{2}\left(P_{e}(\xi)\right)}{\log _{2}(\xi)} \geq \lim _{\xi \rightarrow \infty} \frac{\log _{2}\left(P_{\text {out }}(\xi)\right)}{\log _{2}(\xi)}
$$

Therefore, based again on [112], the following definition for the diversity gain is used that employs the outage probability $P_{\text {out }}(\xi)$ instead of the error one $P_{e}(\xi)$

$$
d(r)=-\lim _{\xi \rightarrow \infty} \frac{\log _{2}\left(P_{\text {out }}(\xi)\right)}{\log _{2}(\xi)}
$$

The following theorem provides the DMT curve of the proposed scheme

Theorem 4.5 The DMT curve of the proposed technique is given by

$$
(M+N-L+1)(1-r(L+1) / L) .
$$

Proof The outage probability for the targeted data rate $R=r \frac{L+1}{L} \log _{2}(\xi)$ is given according to eq.(4.7) by

$$
\begin{aligned}
& P_{\text {out }}=\sum_{K=0}^{N-L}\left(\begin{array}{l}
N \\
K
\end{array}\right)\left(1-e^{-\frac{\xi^{-\left(1-\frac{L+1}{L} r\right)}}{0.5 \gamma_{S R}^{2}}}\right)^{K} e^{-\frac{(N-K) \xi^{-\left(1-\frac{L+1}{L} r\right)}}{0.5 \gamma_{S R}^{2}}} \times \\
& \left(\frac{1}{0.5 \gamma_{S D}^{2}}\right)^{M}\left(\frac{1}{0.5 \gamma_{S D}^{2} \bar{\lambda}_{L}}\right)^{\beta(K)} G_{\beta(K)+M+1, \beta(K)+M+1}^{\beta(K)+M+1,0}\left(\begin{array}{c}
\boldsymbol{\Psi}_{1} \\
\mathbf{\Psi}_{2}
\end{array} \mid, e^{-\xi^{-\left(1-\frac{L+1}{L} r\right)}}\right)+
\end{aligned}
$$

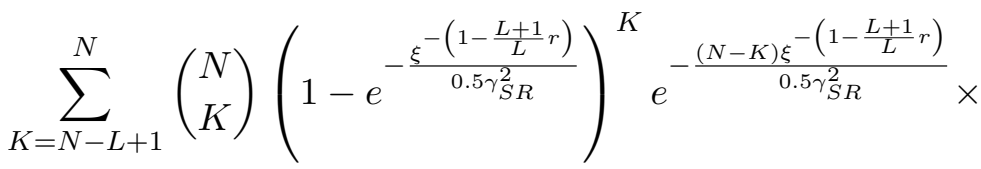

$$
\begin{aligned}
& \left(1-e^{-\frac{\xi^{-\left(1-\frac{L+1}{L} r\right)}}{0.5 \gamma_{S D}^{2}}} \sum_{m=0}^{M-1} \frac{\left(\frac{\xi^{-\left(1-\frac{L+1}{L} r\right)}}{0.5 \gamma_{S D}^{2}}\right)^{m}}{m !}\right),
\end{aligned}
$$

Note that the data rate $r$ is scaled by a factor $(L+1) / L$ due to the fact that the proposed cooperative scheme requires $L+1$ timeslots to transmit $L$ symbols.

Let us now inspect the terms of eq.(4.22) and identify their corresponding order of decay as $\xi \rightarrow \infty$. As it was already mentioned before, both the two distinct sum 
operators include the term $\left(1-e^{-\frac{\xi^{-\left(1-\frac{L+1}{L} r\right)}}{0.5 \gamma_{S R}^{2}}}\right)^{K} e^{-\frac{\xi^{-\left(1-\frac{L+1}{L} r\right)}}{0.5 \gamma_{S R}^{2}}} \cdot$ Now, as $\xi \rightarrow \infty$ we have

$$
\lim _{\xi \rightarrow \infty} e^{-\frac{\xi^{-\left(1-\frac{L+1}{L} r\right)}}{0.5 \gamma_{S R}^{2}}}=1
$$

and

$$
\left(1-e^{-\frac{\xi^{-\left(1-\frac{L+1}{L} r\right)}}{0.5 \gamma_{S R}^{2}}}\right)^{K} \approx\left(0.5 \gamma_{S R}^{2}\right)^{-K} \xi^{-K\left(1-\frac{L+1}{L} r\right)}
$$

where the Taylor series expansion formula of $\left(1-e^{-x}\right)=x+\mathcal{O}\left(x^{2}\right)$ around 0 was used. Moreover according to [82] the outage probability of the SIMO channel can be approximated for the same target rate $R$ as $\xi \rightarrow \infty$ by the following equation

$$
1-e^{-\frac{\xi^{-\left(1-\frac{L+1}{L} r\right)}}{0.5 \gamma_{S D}^{2}}} \sum_{m=0}^{M-1} \frac{\left(\frac{\xi^{-\left(1-\frac{L+1}{L} r\right)}}{0.5 \gamma_{S D}^{2}}\right)^{m}}{m !} \approx\left(0.5 \gamma_{S D}^{2}\right)^{-M} \xi^{-M\left(1-\frac{L+1}{L} r\right)}
$$

It remains to seek for a simplified expression for the term

$$
\begin{aligned}
P_{\text {out }}^{e q(N-K)} & =\left(\frac{1}{0.5 \gamma_{S D}^{2}}\right)^{M}\left(\frac{1}{0.5 \gamma_{S D}^{2} \bar{\lambda}_{L}}\right)^{\beta(K)} \times \\
& G_{\beta(K)+M+1, \beta(K)+M+1}^{\beta(K)+M+1,0}\left(\boldsymbol{\Psi}_{1} \boldsymbol{\Psi}_{2} \mid, e^{-\xi^{-\left(1-\frac{L+1}{L} r\right)}}\right)
\end{aligned}
$$

as $\xi \rightarrow \infty$. By using the results of [117] one can rewrite $P_{\text {out }}^{e q(N-K)}$ as

$$
\begin{aligned}
P_{\text {out }}^{e q(N-K)} & =\frac{1}{\Gamma(1+M+\beta(K))}\left(\frac{\xi^{-\left(1-\frac{L+1}{L} r\right)}}{0.5 \gamma_{S D}^{2}}\right)^{M}\left(\frac{\xi^{-\left(1-\frac{L+1}{L} r\right)}}{0.5 \gamma_{S D}^{2} \bar{\lambda}_{L}}\right)^{\beta(K)} \times \\
& \boldsymbol{\Phi}_{2}^{(2)}\left(M, \beta(K) ; 1+M+\beta(K) ; \frac{-\xi^{-\left(1-\frac{L+1}{L} r\right)}}{0.5 \gamma_{S D}^{2}},-\frac{\xi^{-\left(1-\frac{L+1}{L} r\right)}}{0.5 \gamma_{S D}^{2} \bar{\lambda}_{L}}\right)
\end{aligned}
$$

where $\Gamma(\cdot)$ is the Gamma function and $\boldsymbol{\Phi}_{2}^{(2)}$ is the confluent Lauricella multivariate hypergeometric function defined in [118]-[119]. Now by using the equivalence of the 
latter function with the Appell hypergeometric one [118] we can rewrite eq.(4.27) as

$$
\begin{aligned}
P_{\text {out }}^{e q(N-K)} & =\frac{1}{\Gamma(1+M+\beta(K)) \gamma_{S D}^{2 M} 0.5^{M} \gamma_{S D}^{2 \beta(K)} 0.5^{\beta(K)} \bar{\lambda}_{L}^{\beta K}} \xi^{-(M+\beta(K))\left(1-\frac{L+1}{L} r\right)_{\times}} \\
& \sum_{m=0}^{\infty} \sum_{n=0}^{\infty} \frac{(M)_{m}(\beta(K))_{n}}{m ! n !(1+M+\beta(K))_{m+n}}\left(\frac{\xi^{-\left(1-\frac{L+1}{L} r\right)}}{0.5 \gamma_{S D}^{2}}\right)^{m}\left(\frac{\xi^{-\left(1-\frac{L+1}{L} r\right)}}{0.5 \gamma_{S D}^{2} \bar{\lambda}_{L}}\right)^{n}
\end{aligned}
$$

where $(\alpha)_{k}=\frac{\Gamma(\alpha+k)}{\Gamma(\alpha)}$ is the Pochammer symbol. The outage probability is dominated by the largest exponent of $\xi$ as $\xi \rightarrow \infty$ which is given by setting $m=0$ and $n=0$ in the double infinite series sum of eq.(4.28), that is

$$
P_{\text {out }}^{e q(N-K)} \approx \frac{1}{\Gamma(1+M+\beta(K)) \gamma_{S D}^{2 M} \gamma_{S D}^{2 \beta(K)} 0.5^{M+\beta(K)} \bar{\lambda}_{L}^{\beta(K)}} \xi^{-(M+\beta(K))\left(1-\frac{L+1}{L} r\right)}
$$

Now by plugging eqs.(4.24), (4.25) and (4.29) into eq.(4.22) we have as $\xi \rightarrow \infty$

$$
\begin{aligned}
P_{\text {out }} \approx & \sum_{K=0}^{N-L}\left(\begin{array}{l}
N \\
K
\end{array}\right)\left(0.5 \gamma_{S R}^{2}\right)^{-K} \xi^{-K\left(1-\frac{L+1}{L} r\right)} \times \\
& \frac{1}{\Gamma(1+M+\beta(K)) \gamma_{S D}^{2 M} 0.5^{M} \gamma_{S D}^{2 \beta(K)} 0.5^{\beta(K)} \bar{\lambda}_{L}^{\beta(K)}} \xi^{-(M+\beta(K))\left(1-\frac{L+1}{L} r\right)}+ \\
& \sum_{K=N-L+1}^{N}\left(\begin{array}{l}
N \\
K
\end{array}\right)\left(0.5 \gamma_{S R}^{2}\right)^{-K} \xi^{-K\left(1-\frac{L+1}{L} r\right)}\left(0.5 \gamma_{S D}^{2}\right)^{-M} \xi^{-M\left(1-\frac{L+1}{L} r\right)}
\end{aligned}
$$

It is evident that the outage probability of eq.(4.30) is dominated by the term having the largest exponent of $\xi$. Thus, by inspecting the later equation one can see that the term that dominates the decaying speed of the outage probability of the system as $\xi \rightarrow \infty$ is the

$$
\left(\begin{array}{l}
N \\
K
\end{array}\right)\left(0.5 \gamma_{S R}^{2}\right)^{-K} \xi^{-K\left(1-\frac{L+1}{L} r\right)}\left(0.5 \gamma_{S D}^{2}\right)^{-M} \xi^{-M\left(1-\frac{L+1}{L} r\right)}
$$

one for $K=N-L+1$. Therefore, by combining the previous result with the definition of the DMT (4.20), we obtain the DMT curve of eq.(4.21) and the proof is completed. 


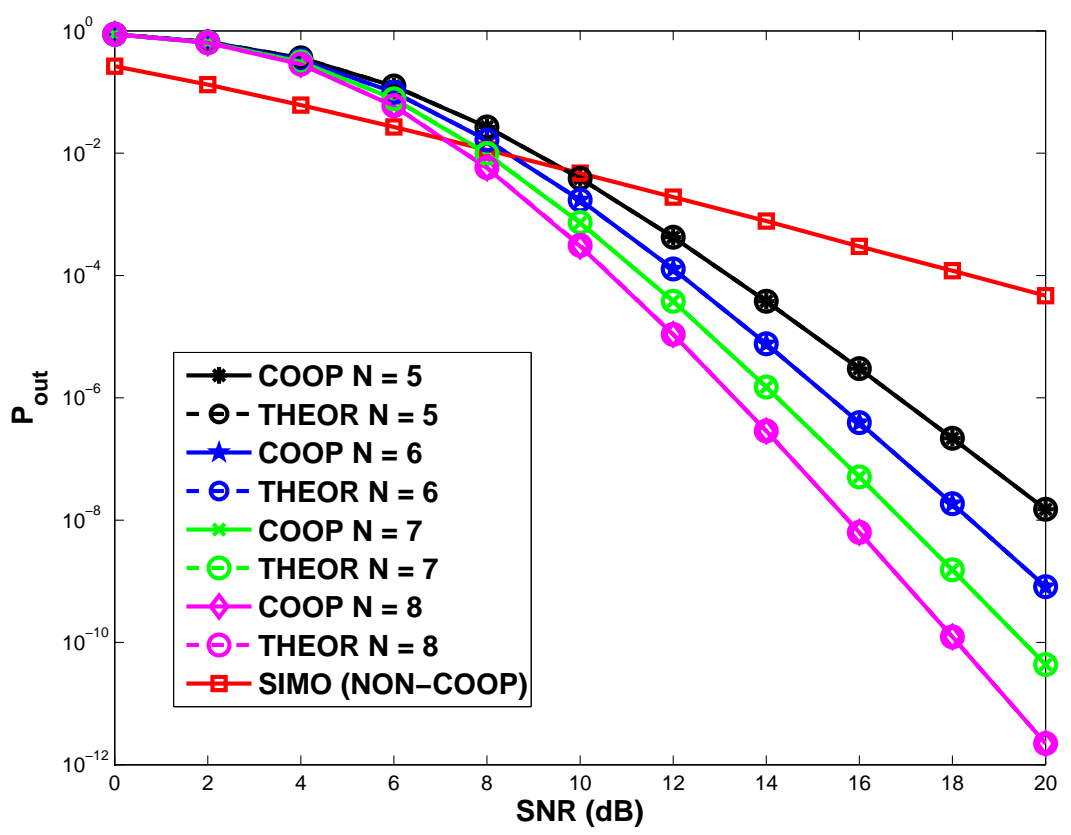

Figure 4-2: Outage probability versus transmit SNR of the non-cooperative (MISO) and the proposed cooperative technique for a different number of relay nodes.

\subsection{Simulations}

In this section representative simulations are provided in order to compare the performance of the proposed scheme with the corresponding non-cooperative SIMO one for different values of the involved parameters. To this end, the outage probability versus the transmit SNR of the corresponding techniques is depicted in the following Figures for a transmission rate of $R=1 \mathrm{bit} / \mathrm{s} / \mathrm{Hz}$ and source transmission power $P_{S}=1$. Note that for a given target rate $R$, the target rate of the proposed cooperative scheme is set to $\frac{L+1}{L} R$ so that the comparison to SIMO performance is fair. We remind that this is due to the half duplex constraint which dictates that the proposed technique requires $L+1$ timeslots to transmit $L$ symbols from the source to the destination. Moreover, note that no comparison to the performance of existing cooperative schemes is done, i.e. the conventional DF protocol of [30], due to the fact that they are designed for single-antenna destinations whereas the proposed scheme requires a multiple antenna destination.

More specifically, in Fig.4-2 the performance of the proposed technique is examined for different number of relay nodes $N=5,6,7$ and 8 . The destination node has 


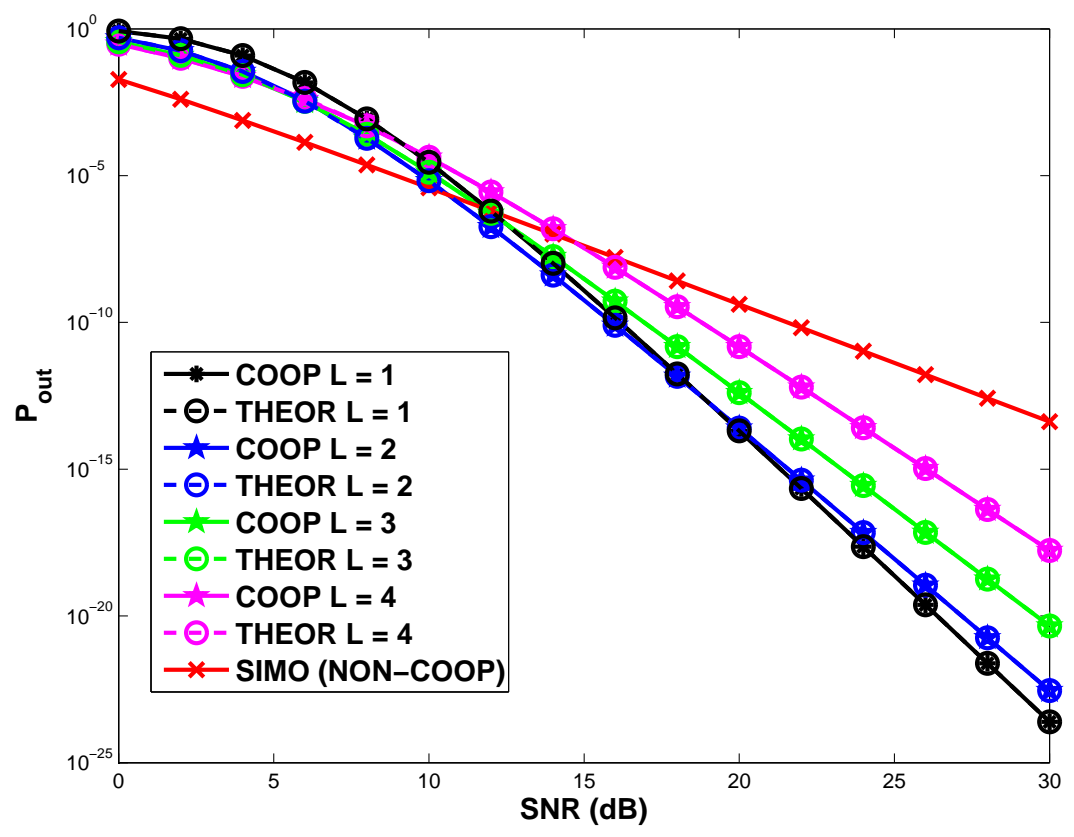

Figure 4-3: Outage probability versus transmit SNR of the non-cooperative (MISO) and the proposed cooperative technique for different values of $L$.

$M=2$ antennas, $L=2$ and $\gamma_{S R}^{2}=\gamma_{S D}^{2}=1$. As it is depicted, in low SNR regimes the proposed scheme achieves worse performance than the non-cooperative SIMO system. This is typical in half duplex cooperative techniques, and it is due to the certain spectrum utilization loss resulted by the half duplex constraint [30]. Contrariwise, for high SNR regimes, the benefits of diversity overtake the costs of the half duplex nature of the proposed scheme and the latter achieves better performance than the one of the non-cooperative system. An increase in the number of relays results in improved performance as it is expected and also proved by the theoretical analysis. In the same figure, the theoretical outage probability of the proposed technique derived in Theorem 4.1 is also plotted. As we can see the theoretical results coincide with the ones of the simulations.

In Fig.4-3 the impact of the block size parameter $L$ on the system's performance is examined. We consider a destination of $M=4$ antennas, $N=6$ relays and $\gamma_{S R}^{2}=\gamma_{S D}^{2}=1$. The performance of the proposed technique is depicted for $L=1,2,3$ and 4. In the same figure, the performance again of the corresponding SIMO of the same number of destination antennas is depicted. Clearly, at the high SNR regime, 


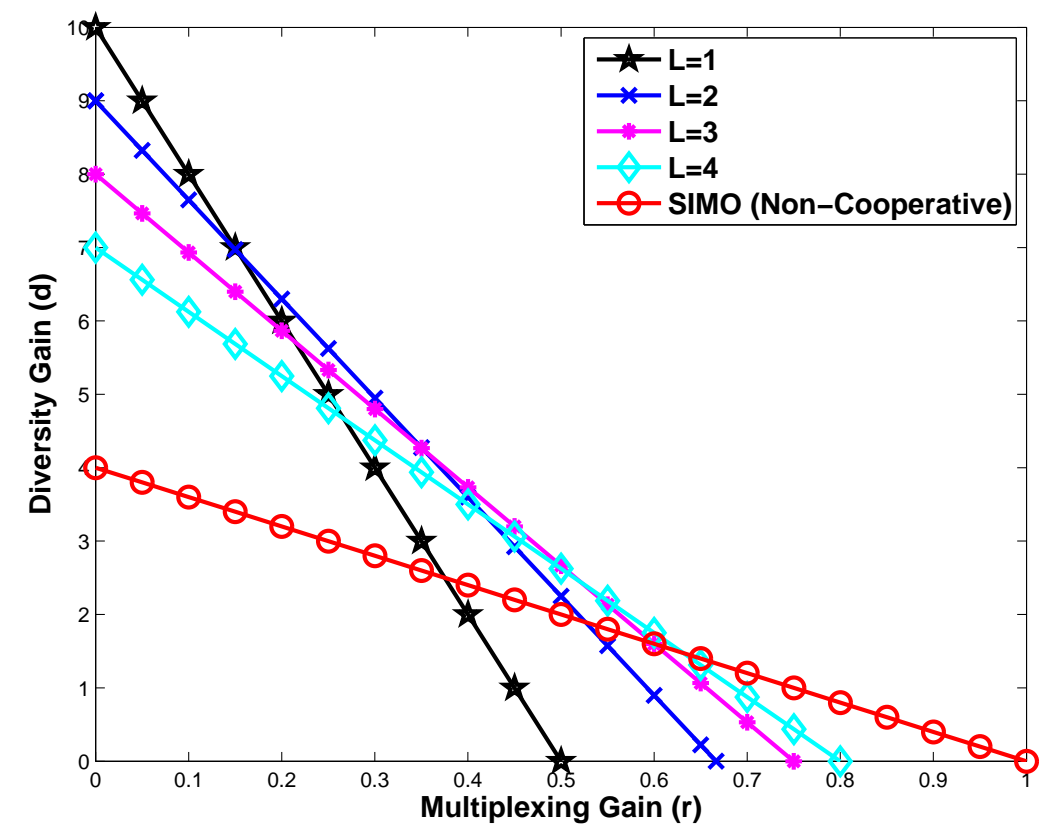

Figure 4-4: DMT curves of the proposed cooperative technique for different values of $L$ and the non cooperative SIMO system

the proposed scheme achieves better performance for any value of $L$. As it is expected, the smaller the value of $L$ the better the performance, since the achieved diversity gain depends on the value of $L$. However, it is interesting to note that this not the case in at the low SNR region. Of course, the performance for any value of $L$ is worse than the one of the non-cooperative SIMO scheme, though the worse performance is achieved for the smaller value of $L$. This is again due to the half-duplex constraint. Lower values of $L$ result in increased targeted rates $\frac{L+1}{L} R$ that must be achieved by the proposed scheme so that the comparison of the its performance to the one of the non cooperative SIMO system to be fair. The latter increased target rates result in their turn in a increased number of outage events between the source-torelays transmission that degrade the overall performance. At any case, the major advantage of the proposed technique is that it can be configured so as to achieve greater multiplexing gains by setting the value of parameter $L$. Note that again the theoretical results of Theorem 4.1 coincide with ones of the simulations. The above conclusions are also verified by the corresponding DMT curves of Fig.4-4.

In Fig.4-5 we examine the impact of the ratio $\frac{\gamma_{S R}^{2}}{\gamma_{S D}^{2}}$ on the performance of the 


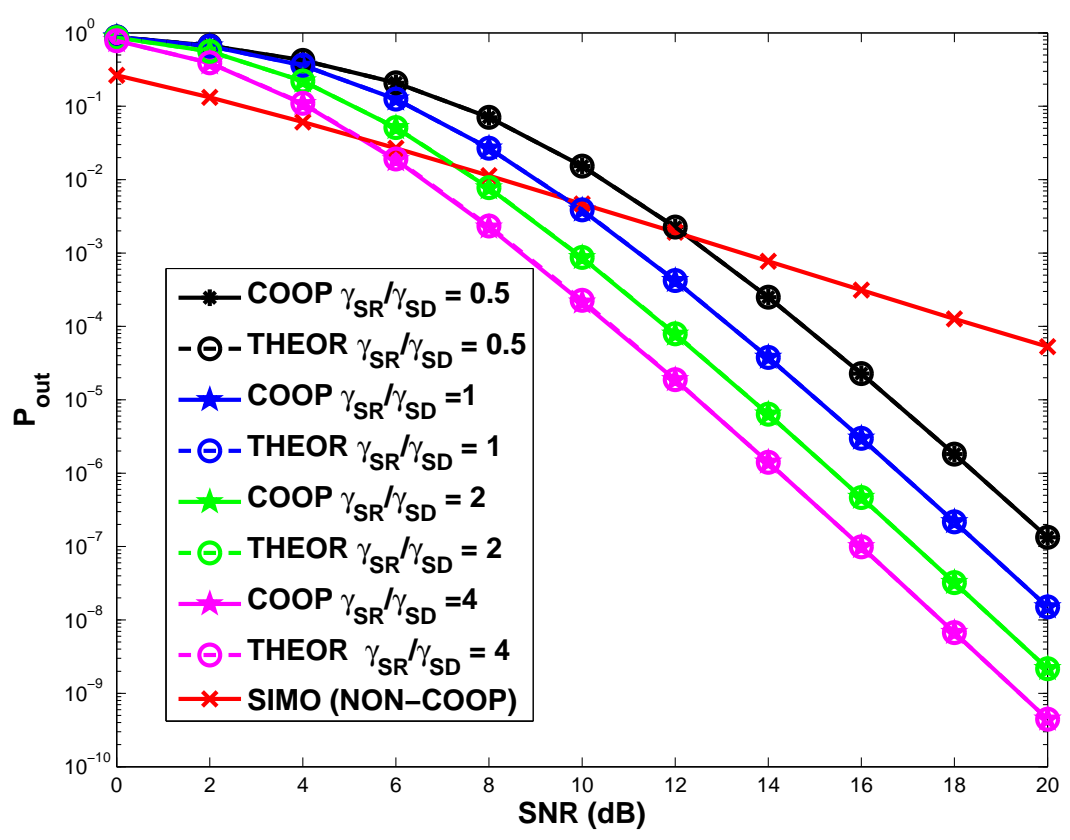

Figure 4-5: Outage probability versus transmit SNR of the non-cooperative (MISO) and the proposed cooperative technique for different values of the ratio $\frac{\gamma_{S R}^{2}}{\gamma_{S D}^{2}}$.

technique. The system has $M=3$ destination antennas, $N=5$ relays and $L=2$ block size. We examine the performance for $\frac{\gamma_{S R}^{2}}{\gamma_{S D}^{2}}=0.5,1,2$ and 4 . In the same figure the performance of the corresponding SIMO system and the theoretical ones are also depicted. It is evident, that as the source-relays channel exhibit better condition the performance is improved as the "weak" part of the system is the sourceto-relays transmission since it lacks of diversity. The latter observation designates also another advantage of the proposed technique. In cellular systems the users can employ the proposed technique with relays that are other users in their close proximity and therefore have channels in relative good condition, so as to improve the uplink performance when they share channels in relative bad condition with the corresponding base station.

\subsection{Conclusion}

In this chapter a new uplink transmission technique of a cooperative system consisted of a single-antenna source, single antenna relay nodes and a multi-antenna destination 
node has been proposed. The new scheme is based on the DF protocol and provide cooperative diversity gain while exhibiting also improved multiplexing gain compared to existing literature approaches. The theoretical outage probability and DMT curve of the proposed scheme was derived for Rayleigh fading channels. Representative simulations verified the performance of the proposed technique and the theoretical results. 


\section{Chapter 5}

\section{Distributed Blind Adaptive Computation of Beamforming Weights for Relay Networks}

\subsection{Introduction}

In this chapter the final research attempt in this dissertation is presented. One of the recent advances in cooperative systems literature is the development of cooperative beamforming techniques, as it was mentioned in Section 1.5. Generally speaking, a beamforming technique has to do with the computation of the weights of a spatial filter so as to optimize a cost function subject to some kind of constraints. For example, in communications systems it is typical to derive the latter weights so as to maximize the received SNR subject to a transmission power constraint or to minimize the transmission power subject to a Quality of service constraint (Qos). Moreover, in case of cognitive radio the beamforming weights of the SU user are the solution to an optimization problem that satisfies extra constraints that concern the PU communication, i.e. interference temperature level to the PU transmissions (underlay approach). Cooperative beamforming techniques differ from their classical counterparts where the array elements are located in a common processing unit, due to the 
distribution of the relays in the space. However, the limited communication resources of the relay nodes, such as battery lifetime, challenges the design of the cooperative techniques, imposing power constraints to the relay transmissions.

In order to solve the above optimization problems, there is the requirement of CSI concerning the source-to-relays and the relays-to destination channels. The aforementioned CSI is usually the exact fading gains or some corresponding second order statics of them. To the best of our knowledge, the majority of the proposed approaches derive the corresponding beamforming weights considering perfect CSI information. Moreover, in cooperative beamforming schemes they usually assume the existence of a fusion center where the required weights are computed. As it is evident, a development of a beamforming scheme in which the perfect CSI estimation assumption does not hold is very challenging when cooperative systems are involved. Our motivation here was to develop a blind and completely decentralized estimation scheme for known cooperative beamforming techniques. The blind term refers to the capability of the technique to estimate the corresponding beamforming weights by using the received signals at the relays, that is without the need for transmission of training symbols.

In more detail, in this chapter, we propose two distributed blind adaptive algorithms which enable the relay cooperation for the computation of the beamforming weights leveraging the computational abilities of the relays, without the need to forward the data to a fusion center [77]. The proposed techniques are extensions of our distributed principal subspace tracking method (DDPM) that it was presented in Section 3.4 where each relay can estimate only the corresponding entry of the principal eigenvector, combining data from its network neighbours. The proposed algorithms are applied to two distributed beamforming schemes for relay networks. In the first scheme, the beamforming vector is computed through minimization of a total transmit power subject to the receiver quality-of-service (QoS) constraint. In the second scheme, the beamforming weights are obtained through maximization of the receiver SNR subject to a total transmit power constraint. Moreover, the proposed algorithms operate blindly, implying that no training data are required to be transmitted to the relays, and adaptively, exhibiting a quite short convergence period. 
The remainder of the chapter is organized as follows. In Section 5.2, we present the problem formulation and the distributed beamforming schemes. In Section 5.3 the proposed distributed blind adaptive algorithms for the computation of the beamforming weights are derived. Simulation results are provided in Section 5.4, followed by concluding remarks in Section 5.5.

\subsection{Problem Formulation}

\subsubsection{System Model}

Let us consider a wireless network which consists of a transmitter, a receiver, and $K$ relay nodes. The network operates over flat fading channels in the links transmitterrelays and relays-receiver. It is assumed that synchronization between all the terminals is perfect, and there is no direct link between the transmitter and the receiver. Furthermore, we consider the AF protocol with half mode duplexing, i.e. the transmission cycle consists of two time slots, where during the first cycle the transmitter broadcast the signal to the relays, while at the second one the relays amplify and forward the received signals to the destination.

Hence, during the first cycle of the AF protocol, the transmitted signal $s_{0}$ is broadcasted to the relays with power $P_{0}$. Then, each relay $r_{k}$, with $k=1, \ldots, K$, receives

$$
y_{k}=f_{k} s_{0}+\eta_{k}
$$

where $f_{k}$ is the channel from the transmitter to the $k$-th relay. The multiple-input single-output (MISO) from the transmitter to the relays is denoted as $\mathbf{f}=\left[f_{1}, \ldots, f_{K}\right]^{T}$ $\in \mathbb{C}^{K}$ and $\eta_{k}$ is the complex zero-mean additive-white Gaussian noise (AWGN) of the $k$-th relay with variance $\sigma_{\eta}^{2}$, equal for all relays.

During the second cycle, the $k$-th relay amplifies $y_{k}$ with the beamforming weight coefficient $w_{k}$. Therefore, the $K$ relays forward their signal to the destination, where 
the following signal is received

$$
z=\underbrace{\sum_{k=1}^{K}\left(w_{k} f_{k} g_{k}\right) s_{0}}_{\text {signal component, } z_{s}}+\underbrace{\sum_{k=1}^{K}\left(w_{k} g_{k}\right) \eta_{k}+v}_{\text {total noise }, z_{n}}
$$

where $g_{k}$ is the channel form the $k$-th relay to the receiver and $v$ is the zero-mean AWGN of the destination with variance $\sigma_{v}^{2}$.

\subsubsection{Distributed Beamforming Schemes}

In this subsection, we review two different schemes which are used in order to compute the beamforming weight vector.

\section{Total Relay Power Minimization Under QoS Constraint}

Let us consider the distributed AF beamforming problem that provides the relay beamforming weights by minimizing the total relay transmitted power $P$ subject to (s.t.) QoS constraint at the destination. That means that the SNR, which is defined as the ratio of the signal power $P_{s}$ to the noise power $P_{n}$ at the destination, is required to be larger than a certain predefined threshold $\gamma>0$. Thus, the total relay transmit power $P$ can be obtained as

$$
P=\sum_{k=1}^{K} \mathbb{E}\left\{\left|y_{k} w_{k}\right|^{2}\right\}=\sum_{k=1}^{K}\left|w_{k}\right|^{2} \mathbb{E}\left\{\left|y_{k}\right|^{2}\right\}=\mathbf{w}^{H} \mathbf{D} \mathbf{w}
$$

where $\mathbf{D} \triangleq \operatorname{diag}\left(\left[\left|f_{1}\right|^{2} \ldots\left|f_{K}\right|^{2}\right)+\sigma_{\eta}^{2} \mathbf{I}, \mathbf{w}=\left[w_{1}, \ldots, w_{K}\right]^{T} \in \mathbb{C}^{K}\right.$ is defined as the beamforming vector. The noise power $P_{n}$ can be obtained as

$$
\begin{aligned}
P_{n} & =\mathbb{E}\left\{\left|z_{n}\right|^{2}\right\}=\mathbb{E}\left\{\sum_{k=1}^{K}\left|w_{k} g_{k} \eta_{n}\right|^{2}\right\}+\mathbb{E}\left\{|v|^{2}\right\} \\
& =\mathbf{w}^{H} \mathbf{G} \mathbf{w}+\sigma_{v}^{2}
\end{aligned}
$$


where $\mathbf{G} \triangleq \sigma_{\eta}^{2} \mathbf{g} \mathbf{g}^{H}$ and $\mathbf{g}=\left[g_{1}, \ldots, g_{K}\right]^{T} \in \mathbb{C}^{K}$ is defined as the MISO channel from the relays to destination receiver. The signal power $P_{s}$ can be obtained as

$$
\begin{aligned}
P_{s} & =\mathbb{E}\left\{\left|z_{s}\right|^{2}\right\}=\sum_{k, l=1}^{K}\left|w_{k} w_{l}^{*} f_{k} f_{l}^{*} g_{k} g_{l}^{*}\right|^{2} \mathbb{E}\left\{\left|s_{0}\right|^{2}\right\} \\
& =\mathbf{w}^{H} \mathbf{R} \mathbf{w}
\end{aligned}
$$

where $\mathbf{R}$ is the correlation matrix of the vector $\mathbf{h}=\left[f_{1} g_{1} \ldots f_{K} g_{K}\right]^{T}=\mathbf{f} \odot \mathbf{g}$, i.e. $\mathbf{R} \triangleq \mathbf{h h}^{H}=(\mathbf{f} \odot \mathbf{g})(\mathbf{f} \odot \mathbf{g})^{H}=\mathbf{f f}^{H} \odot \mathbf{g g}^{H}$ and $\odot$ represents the element-wise SchurHadamard product. Based on (5.3), (5.4) and (5.5), the beamforming weight computation of this scheme is equivalent to the following optimization problem

$$
\min _{\mathbf{w}} \mathbf{w}^{H} \mathbf{D w} \text { s.t. } \frac{\mathbf{w}^{H} \mathbf{R} \mathbf{w}}{\mathbf{w}^{H} \mathbf{G} \mathbf{w}+\sigma_{v}^{2}} \geq \gamma
$$

Based on the methodology of [50] and [52], the solution of (5.6) is given by the following closed form expression

$$
\mathbf{w}_{o p t}^{1}=\beta \mathbf{D}^{-1 / 2} \mathcal{P}_{1}(\mathbf{Q})
$$

where

$$
\mathbf{Q}=\mathbf{D}^{-1 / 2}(\mathbf{R}-\gamma \mathbf{G}) \mathbf{D}^{-1 / 2}
$$

and

$$
\beta=\left(\frac{\gamma \sigma_{v}^{2}}{\mathcal{P}_{1}(\mathbf{Q}) \mathbf{Q} \mathcal{P}_{1}(\mathbf{Q}}\right)^{1 / 2}
$$

where $\mathcal{P}_{1}(\cdot)$ represents the principal eigenvector of a matrix. We must note that, the feasibility of the constraint optimization problem of (5.6) depends on the values of the parameter $\gamma$. Thus, for all the values of $\gamma$ that lead to a negative semidefinite $\mathbf{Q}$, the problem becomes infeasible. 


\section{QoS Maximization Under Total Power Constraint}

In the second scheme, we consider the distributed AF beamforming problem that provides the relay beamforming weights by maximizing the SNR at the destination subject to a constraint on the maximum allowable total transmit power of the relays $P_{\max }$. Equivalently to the previous scheme, based on (5.3), (5.4) and (5.5), the optimization problem in this case can be written as

$$
\max _{\mathbf{w}} \frac{\mathbf{w}^{H} \mathbf{R} \mathbf{w}}{\mathbf{w}^{H} \mathbf{G} \mathbf{w}+\sigma_{v}^{2}} \text { s.t. } \mathbf{w}^{H} \mathbf{D} \mathbf{w} \leq P_{\max }
$$

Following the methodology of [50] and [52], the solution of (5.10) is given by the closed form expression

$$
\mathbf{w}_{\text {opt }}^{2}=\mathbf{D}^{-1 / 2} \mathcal{P}_{1}\left\{\left(\sigma_{v}^{2} \mathbf{I}+\tilde{\mathbf{G}}\right)^{-1} \tilde{\mathbf{R}}\right\}
$$

where $\tilde{\mathbf{R}}=\mathbf{D}^{-1 / 2} \mathbf{R} \mathbf{D}^{-1 / 2}$ and $\tilde{\mathbf{G}}=\mathbf{D}^{-1 / 2} \mathbf{G D}^{-1 / 2}$.

\subsection{Distributed Blind Estimation Scheme of the Beamforming Vector}

As it is evident from the previous section, the involved matrices $\mathbf{D}, \mathbf{R}$ and $\mathbf{G}$ must be known in order to derive the optimal beamforming vector for each one of the two problems under consideration. In a centralized approach, the aforementioned information should be gathered to a fusion center in order to compute the beamforming weights which are then fed back to the relay nodes. According to the previous section, the optimal beamforming vectors for each problem require eigenvalue decomposition computations that can be of high complexity for systems that involve large number of relays.

In this section, two new algorithms are developed that enable the relays to estimate the corresponding beamforming weights in a distributed manner. At first, it is shown how the beamforming vectors can be updated in a blind manner via em- 
ploying adaptive subspace tracking techniques of the second order statistics of the data received at the relays. Then, a distributed version of the previous technique is described for each one of the two beamforming schemes. In order to develop the aforementioned distributed schemes asimilar approach to the one of the Distributed Data Projection Method (DDPM) of Table 3.II was followed

In order for the relays to be able to estimate the beamforming weights, an amount of information is required to be transmitted from the source and the destination nodes to the relays. Therefore, it is assumed that the source and the destination nodes transmit data in two orthogonal periods of $N$ symbols each one, denoted as $x^{s}$ and $x^{d}$ respectively, that are i.i.d. with $\mathbb{E}\left\{\left|x_{n}^{s}\right|^{2}\right\}=\mathbb{E}\left\{\left|x_{n}^{d}\right|^{2}\right\}=1$. The orthogonality of the transmissions is achieved by employing a time division approach (TDMA). Thus the received signals at the relays in the two transmission phases are given by

$$
\begin{aligned}
& \mathbf{y}_{n}^{s}=\mathbf{f} x_{n}^{s}+\mathbf{z}_{n}^{s}, \text { for } 1 \leq n \leq N \\
& \mathbf{y}_{n}^{d}=\mathbf{g} x_{n}^{d}+\mathbf{z}_{n}^{d}, \text { for } N+1 \leq n \leq 2 N,
\end{aligned}
$$

where $\mathbf{y}_{n}^{s}$ and $\mathbf{y}_{n}^{d}$ are the received data at the relays during the source and the destination transmissions, respectively, $\mathbf{f}$ and $\mathbf{h}$ are the source to relays and the destination to relays channels in vector form and $\mathbf{z}_{n}^{s}$ and $\mathbf{z}_{n}^{d}$ are independent complex AWGN variables with zero-mean and the same variance equal to $\sigma_{z}^{2}$.

In can be easily verified that the following equations hold

$$
\begin{aligned}
& \mathbb{E}\left\{\left(\mathbf{y}_{n}^{s} \odot \mathbf{y}_{n}^{d}\right)\left(\mathbf{y}_{n}^{s} \odot \mathbf{y}_{n}^{d}\right)^{H}\right\}=\mathbf{R} \\
& \operatorname{diag}\left(\mathbb{E}\left\{\mathbf{y}_{n}^{s}\left(\mathbf{y}_{n}^{s}\right)^{H}\right\}\right)=\mathbf{D}+\sigma_{z}^{2} \mathbf{I}_{K} \\
& \mathbb{E}\left\{\mathbf{y}_{n}^{d}\left(\mathbf{y}_{n}^{d}\right)^{H}\right\}=\mathbf{g g}^{H}+\sigma_{z}^{2} \mathbf{I}_{K}=\frac{1}{\sigma_{n}^{2}} \mathbf{G}+\sigma_{z}^{2} \mathbf{I}_{K} .
\end{aligned}
$$

Therefore, the matrices $\mathbf{D}, \mathbf{R}$ and $\mathbf{G}$ can be obtained blindly, based on the second order statistics of relays' received data. 


\subsubsection{The Power Minimization Problem}

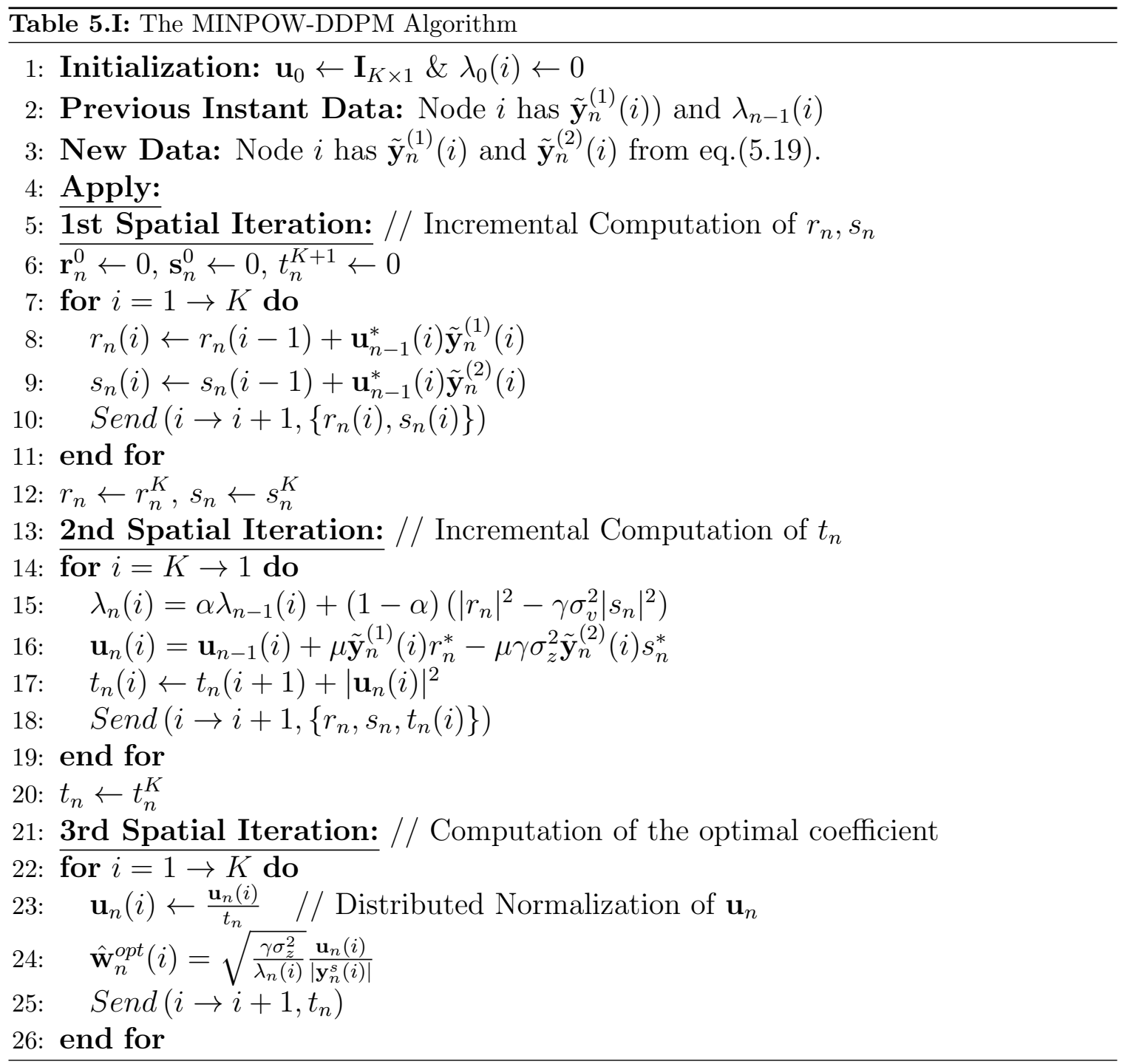

According to (5.7)-(5.8), the optimal beamforming vector requires the computation of the maximum eigenvalue and its corresponding eigenvector of matrix $\mathbf{Q}$. It can be easily verified that the latter matrix is Hermitian and so the DDPM can be applied to track the required components. Let us assume that at each time index $n$ the received data at the relays from the source and the destination transmissions are given by equations (5.12) and (5.13) respectively.

By using the linear properties of the Schur-Hadamard product, the definition of the inverse of a diagonal matrix and the DPM eqs.(3.8)-(3.11), it is easy to show that the following equations can be used to track the desired principal components at the 
$i$-th node

$$
\begin{aligned}
& \mathbf{u}_{n}(i)=\mathbf{u}_{n-1}(i)+\mu \tilde{\mathbf{y}}_{n}^{(1)}(i) r_{n}^{*}-\mu \gamma \sigma_{z}^{2} \tilde{\mathbf{y}}_{n}^{(2)}(i) s_{n}^{*} \\
& \lambda_{n}(i)=\alpha \lambda_{n-1}(i)+(1-\alpha)\left(\left|r_{n}\right|^{2}-\gamma \sigma_{z}^{2}\left|s_{n}\right|^{2}\right)
\end{aligned}
$$

where

$$
\begin{gathered}
\tilde{\mathbf{y}}_{n}^{(1)}(i)=\frac{\mathbf{y}_{n}^{s}(i) \mathbf{y}_{n}^{d}(i)}{\left|\mathbf{y}_{n}^{s}(i)\right|}, \quad \tilde{\mathbf{y}}_{n}^{(2)}(i)=\frac{\mathbf{y}_{n}^{d}(i)}{\left|\mathbf{y}_{n}^{s}(i)\right|}, \\
r_{n}=\sum_{i=1}^{K} \mathbf{u}_{n-1}^{*}(i) \tilde{\mathbf{y}}_{n}^{(1)}(i), \quad s_{n}=\sum_{i=1}^{K} \mathbf{u}_{n-1}^{*}(i) \tilde{\mathbf{y}}_{n}^{(2)}(i) .
\end{gathered}
$$

The complete version of the DDPM algorithm for the the total relay power minimization (MINPOW-DDPM), is given in Table 5.I.

\subsubsection{SNR Maximization Problem}

According to (5.11), the computation of the optimal beamforming vector that maximizes the SNR requires the estimation of the first principal eigenvector of matrix $\mathbf{B}=\boldsymbol{\Phi}^{-1} \tilde{\mathbf{R}}=\left(\sigma_{z}^{2} \mathbf{I}+\tilde{\mathbf{G}}\right)^{-1} \tilde{\mathbf{R}}$, where $\boldsymbol{\Phi}=\left(\sigma_{z}^{2} \mathbf{I}+\tilde{\mathbf{G}}\right)$. The matrix $\mathbf{B}$ in general is not symmetric and therefore the DDPM algorithm cannot be applied directly to track the corresponding eigenvector. Therefore, in the next, we transform the problem into a suitable expression for DDPM application. From the characteristic equation of the matrix $\mathbf{B}$ we have

$$
\boldsymbol{\Phi}^{-1} \tilde{\mathbf{R}} \mathbf{u}=\lambda \mathbf{u} \Rightarrow \tilde{\mathbf{R}} \Phi^{-1}(\boldsymbol{\Phi} \mathbf{u})=\lambda(\boldsymbol{\Phi} \mathbf{u}) \Rightarrow \tilde{\mathbf{R}} \Phi^{-1} \tilde{\mathbf{u}}=\lambda \tilde{\mathbf{u}}
$$

where $\lambda$ and $\mathbf{u}$ are an eigenvalue and the corresponding eigenvector of matrix $\mathbf{B}$, while $\tilde{\mathbf{u}}=\boldsymbol{\Phi} \mathbf{u}$, is an eigenvector of matrix $\tilde{\mathbf{R}} \Phi^{-1}$. By using the Lemma 1 of [120], the principal eigenvector of matrix $\tilde{\mathbf{R}} \Phi^{-1}$ is given by

$$
\tilde{\mathbf{u}}_{1} \triangleq \mathcal{P}_{1}\left\{\tilde{\mathbf{R}} \boldsymbol{\Phi}^{-1}\right\}=\boldsymbol{\Phi}^{1 / 2} \mathcal{P}_{1}\left\{\boldsymbol{\Phi}^{-1 / 2} \tilde{\mathbf{R}} \boldsymbol{\Phi}^{-1 / 2}\right\}
$$

where the matrix $\boldsymbol{\Phi}^{-1 / 2} \tilde{\mathbf{R}} \Phi^{-1 / 2}$ is symmetric, and hence, the DDPM algorthm can be used in order to compute its principal components. Therefore, once $\tilde{\mathbf{u}}_{1}$ has been 
estimated using the DDPM, the computation of the desired eigenvector $\mathbf{u}_{1}$ can be obtained by $\mathbf{u}_{1}=\boldsymbol{\Phi}^{-1} \tilde{\mathbf{u}}_{1}=\boldsymbol{\Phi}^{-1 / 2} \mathcal{P}_{1}\left\{\boldsymbol{\Phi}^{-1 / 2} \tilde{\mathbf{R}} \Phi^{-1 / 2}\right\}$. Note that the computation of the desired subspace requires matrix $\boldsymbol{\Phi}^{-1 / 2}$. Given that $\boldsymbol{\Phi}=\sigma_{z}^{2} \mathbf{I}+\sigma_{z}^{2} P_{\max } \tilde{\mathbf{y}}_{n}^{(2)}\left(\tilde{\mathbf{y}}_{n}^{(2)}\right)^{H}$ and using some algebraic manipulations, matrix $\boldsymbol{\Phi}^{-1 / 2}$ can be computed as follows

$$
\boldsymbol{\Phi}^{-1 / 2}=\frac{1}{\sigma_{z}^{2}} \mathbf{I}_{K \times K}+\beta_{n} \tilde{\mathbf{y}}_{n}^{(2)}\left(\tilde{\mathbf{y}}_{n}^{(2)}\right)^{H}
$$

where $\beta_{n}=\frac{1}{\sqrt{P_{\max }}\left\|\tilde{\mathbf{y}}_{n}^{(2)}\right\|^{2}}\left(\frac{1}{\sqrt{1+\left\|\tilde{\mathbf{y}}_{n}^{(2)}\right\|^{2}}}-1\right)$. Now if we apply the approximation of $\tilde{\mathbf{R}}$ by $\hat{\mathbf{R}}=\tilde{\mathbf{y}}_{n}^{(1)} \tilde{\mathbf{y}}_{n}^{(1)^{H}}$ and eq.(5.22) in $\boldsymbol{\Phi}^{-1 / 2} \tilde{\mathbf{R}} \boldsymbol{\Phi}^{-1 / 2}$ and by using the DPM eqs.(3.8)(3.11) we can show after some calculations that the following equations can be used to update adaptively the principal component of matrix $\mathbf{B}$

$$
\mathbf{u}_{n}(i)=\mathbf{u}_{n-1}(i)+\frac{\mu}{\sigma_{z}^{2}} \delta_{n}(i)
$$

where

$$
\delta_{n}(i)=\tilde{\mathbf{y}}_{n}^{(1)}(i) r_{n}^{*}+d_{n} \tilde{\mathbf{y}}^{(2)}(i) r_{n}^{*}+d_{n}^{*} \tilde{\mathbf{y}}^{(1)}(i) s_{n}^{*}+\left|d_{n}\right|^{2} \tilde{\mathbf{y}}_{n}^{(2)}(i) s_{n}^{*}
$$

and $\tilde{\mathbf{y}}_{n}^{(1)}(i)$ and $\tilde{\mathbf{y}}_{n}^{(2)}(i)$ are given by (5.19).

The complete version of the DDPM algorithm for SNR maximization (MAX-SNR) is given in Table 5.II.

\subsection{Simulations}

In this section some indicative simulations are presented. The performance of the proposed distributed adaptive beamforming schemes is compared to the one of the centralized solutions that provide the optimal beamforming vectors, assuming perfect channel state information at the relay nodes. We consider a cooperative system of $K=4$ relays. All the involved nodes are employing BPSK modulation and the source and destination nodes transmission power is $P=1$. At first, the case of static channels is considered. The source and the destination nodes transmit in orthogonal timeslots $N=1000$ symbols with each one of them at $S N R=20 d B$. The relay nodes apply the 


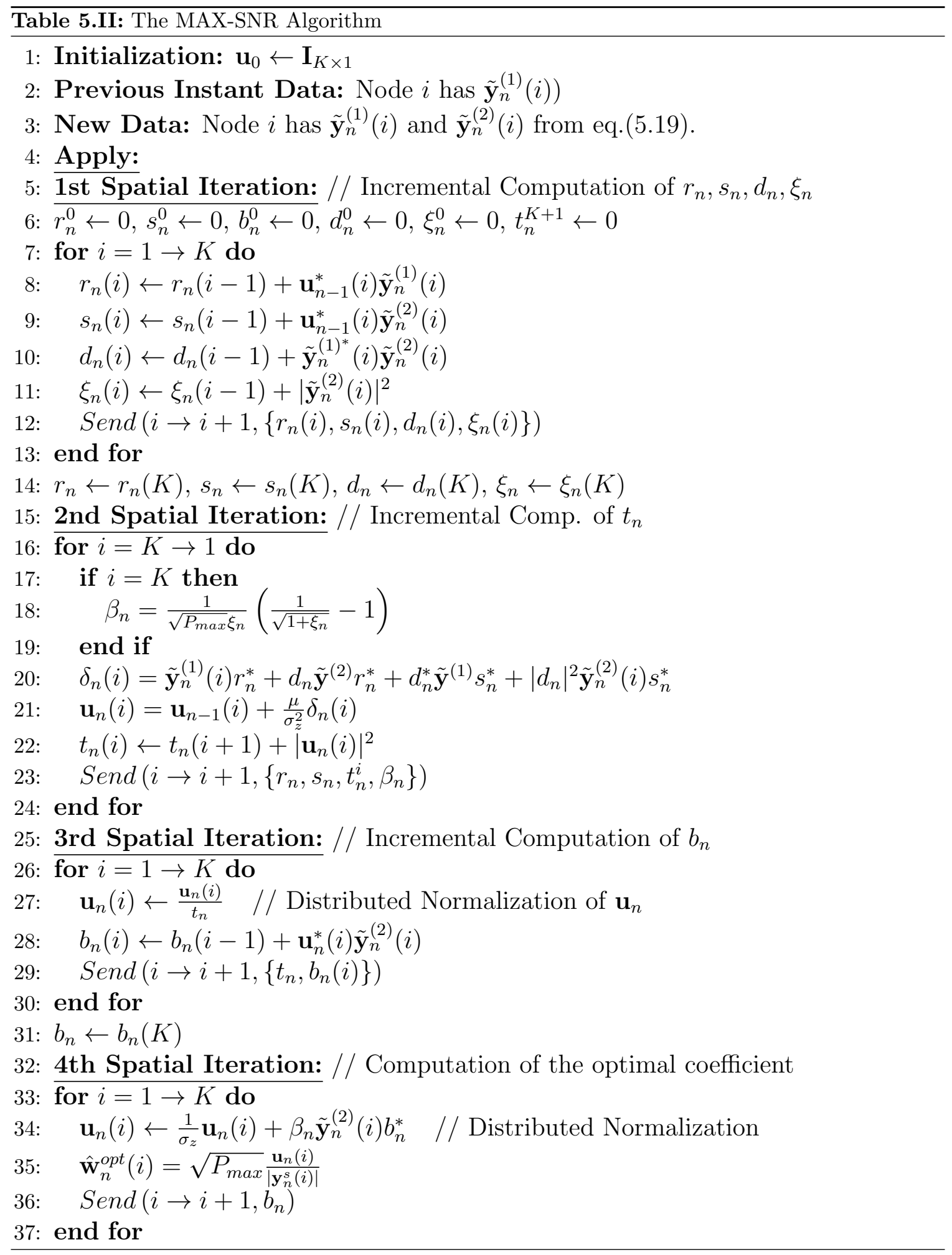


proposed adaptive approaches of Table 5.I and II during the interval of the $N$ symbol periods in order to compute the corresponding optimal beamforming vectors. Both the source-to-relays and relays-to-destination channel gains are modelled as $\mathcal{C} N(0,1)$ and $\mu=0.1$ and $\alpha=0.98$.

In Figure 5-1 the minimum achieved power (a) and the maximum achieved SNR (b) are depicted. As it shown the adaptive beamforming methods exhibit very close performance to the exact beamforming solutions.

In Figure 5-2, the same metrics are depicted, though now time varying-channels are assumed. All the channel taps are produced by a Jakes fading model of Doppler spread equal to $f_{d} T=10^{-4}$ and $\alpha=0.8$. In the beginning, the source and the destination nodes transmit in orthogonal timeslots $N=1000$ symbols, so as the distributed beamforming methods to reach their steady state. Then, the transmission of the source data can start, though the transmission stops periodically when the destination sends some data to the relays in order the latter to update the beamforming coefficients. The performance of the adaptive technique is depicted when the $1 \%$, $5 \%$ and $10 \%$ of the transmission bandwidth is used for feedback data. In the same figure the performance of the optimal beamforming coefficients is depicted. As it is shown, the adaptive methods achieve close performance to the optimal solutions even for very small amount of feedback data.

\subsection{Conclusion}

In this chapter novel de-centralized adaptive methods for the computation of the optimal relay beamforming weights were proposed that minimize the required relays' transmission power or maximize the destination's received SNR in AF-based cooperative systems. The proposed approaches require no fusion center, distribute equally the computational overhead among the relay nodes and function in a completely blind manner, since they require no training data to be transmitted. Moreover, they achieve close performance to the one of the optimal beamforming solutions as it was verified by indicative simulations for static and time-varying channels. The impact 

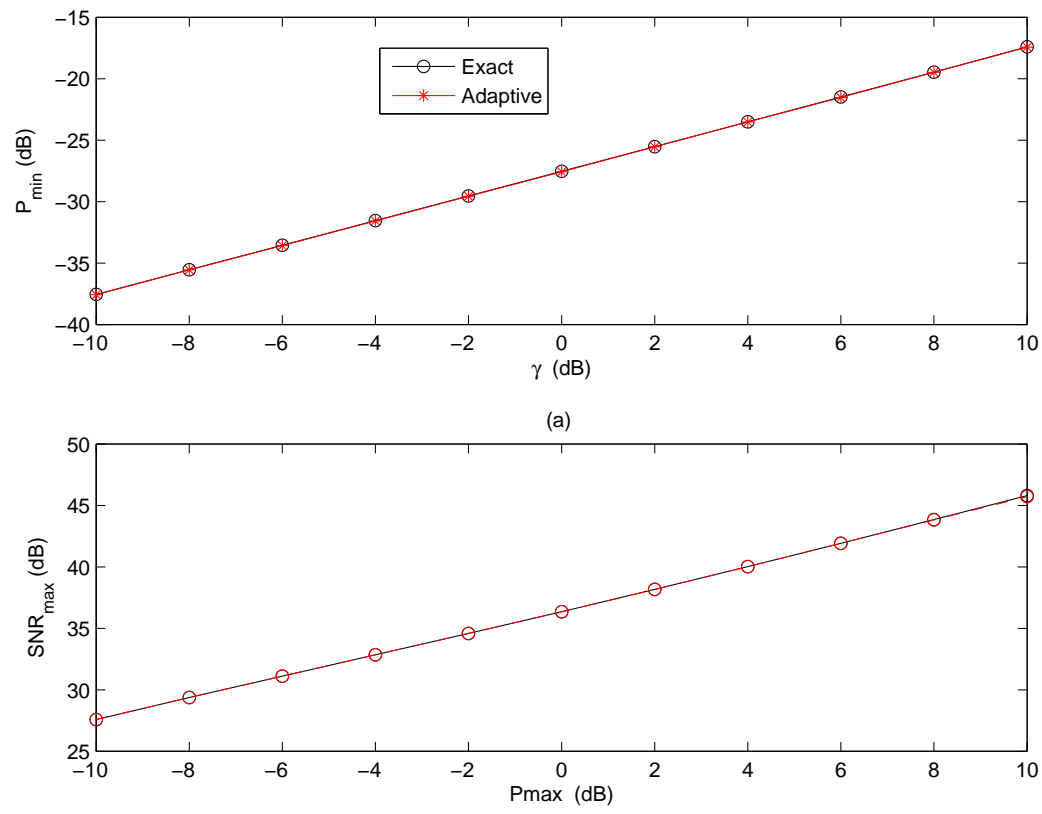

(b)

Figure 5-1: Minimum required Power versus $\gamma$
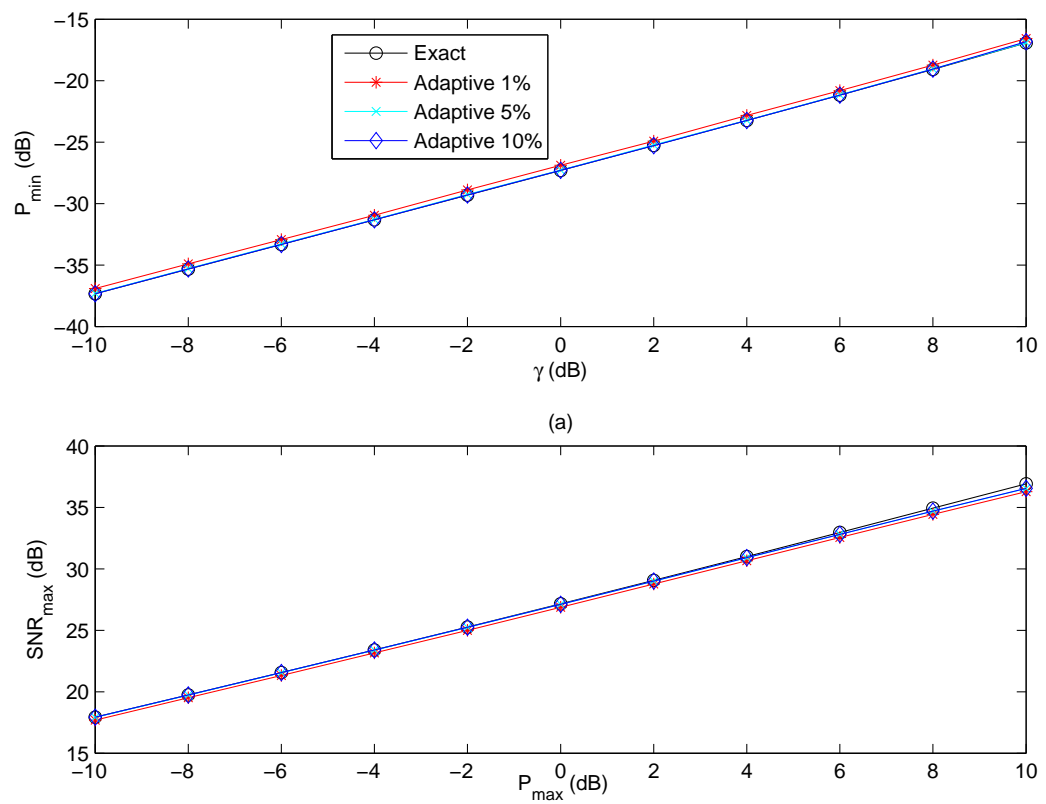

(b)

Figure 5-2: Maximum achieved SNR versus $P_{\max }$

of imperfect synchronization and relay-to-relay communication on the performance of the proposed technique along with the development of schemes that reduce the required communication overheard is currently under study. 


\section{Chapter 6}

\section{Conclusions and Open Issues}

In this chapter the results of the present dissertation are summed up and a reference to possible research extensions is given.

In Chapter 2 a Blind OIA technique for Cognitive Radio systems was derived. Unlike the existing approach, the new one enables the SU to compute blindly the required CSI. The performance of the proposed technique under perfect CSI was theoretically studied by computing close approximations of the PU and the SU ergodic capacities. The performance of the new technique was also studied under imperfect CSI, and lower bounds on the SU capacity were obtained along with some other useful theoretical results. In general, the aim of this work was to develop an efficient and practical interweave technique so as to exploit the spatial spectrum holes that may exist in multi-antenna CR systems. To the best of our knowledge, it is the first time this subject was studied in such depth since the majority of similar approaches derive the CR schemes assuming perfect CSI at least at the SU ends. Moreover, they lack of detailed performance analysis. The proposed approach achieved also better performance in cases where the number of antennas at the SU ends where more than corresponding ones at the PU ends.

Open issues: An interesting open research matter is how the complete scheme and its corresponding performance analysis can be extended to more typical IA scenarios

where there are more than two users that seek in general a way to align their signals to orthogonal subspaces at the corresponding receivers. 
In Chapter 3 cooperative decentralized adaptive versions of the well-known EBSS techniques were developed for multi-antenna cognitive receivers. The proposed techniques offer low complexity and improved performance especially in cases when time varying channels are involved and/or continuous spectrum monitoring is applied (dual radio architecture). Moreover due to their decentralized nature they exhibit reduced transmission power consumption during the sensing period and provide robustness against node and link failures. In order to compute the decision thresholds for each one of the adaptive test statistics, close approximations for the associated distribution functions were derived. A novel distributed subspace tracking method was also derived as a constituent part of the proposed decentralized EBSS techniques. The new distributed ST method enable the SUs to track jointly the subspace of their received signals in a completely decentralized manner.

Open issues: Important issues here arise that are common in the cooperative spectrum sensing literature. Even in cases where a fusion center is involved, the majority of the spectrum schemes assume analogue communication over ideal communication channels. A similar assumption is done also in the distributed estimation literature, where the information flow among the nodes is assumed to be done again over ideal communication channels. Moreover, in the decentralized approaches there are several matters that arise and should be studied in a more general context, such as: How the technique under consideration behaves to link and node failures? What if a new node is added to the network? Do synchronization errors affect the performance of the technique? Thus, it is of great interest to develop more realistic and robust spectrum sensing or distributed estimation techniques.

In Chapter 4 a new uplink transmission technique of a cooperative system consisted of a single-antenna source, single antenna relay nodes and a multi-antenna destination node has been proposed. The new scheme is based on the DF protocol and provide cooperative diversity gain while exhibiting also improved multiplexing gain compared to existing literature approaches. The theoretical outage probability and DMT curve of the proposed scheme was derived for Rayleigh fading channels. Representative simulations verified the performance of the proposed technique and 
the theoretical results.

Open issues: An open research matter here is the extension of the technique to an overlay $\mathrm{CR}$ one that optimizes both the SU and the PU transmissions.

In Chapter 5 novel de-centralized adaptive methods for the computation of the optimal relay beamforming weights were proposed that minimize the required relays' transmission power or maximize the destination's received SNR in AF-based cooperative systems. The proposed approaches require no fusion center, distribute equally the computational overhead among the relay nodes and function in a completely blind manner, since they require no training data to be transmitted. Moreover, they achieve close performance to the one of the optimal beamforming solutions as it was verified by indicative simulations for static and time-varying channels.

Open issues: Here again as open research issues are the development of more realistic and robust distributed estimation techniques. Moreover, an extension to an an overlay CR technique is also possible. Finally, it is challenging to extend the proposed technique to the case where the relays have individual power constraints resulting in solutions for the beamforming vector that do not admit close form. 


\section{Bibliography}

[1] J. Mitola, "Cognitive Radio - An Integrated Agent Architecture for Software Defined Radio," Ph.d. dissertation, Royal Institute of Technology (KTH), Kista, Sweden, May 2000.

[2] FCC Spectrum Policy Task Force, "Report of the spectrum efficiency group," Tech. Rep. 02-135, Nov. 2002.

[3] S. Haykin, "Cognitive radio: brain-empowered wireless communications," IEEE Journal on Selected Areas in Communications, vol. 23, no. 2, pp. 201 - 220, Feb. 2005.

[4] M. Gastpar, "On capacity under receive and spatial spectrum-sharing constraints," IEEE Trans. on Information Theory, vol. 53, no. 2, pp. 471-487, 2007.

[5] A. Ghasemi and E. Sousa, "Capacity of fading channels under spectrumsharing constraints," in IEEE International Conference on Communications, ICC, vol. 10, 2006, pp. 4373-4378.

[6] H. Suraweera, J. Gao, P. Smith, M. Shafi, and M. Faulkner, "Channel capacity limits of cognitive radio in asymmetric fading environments," in IEEE International Conference on Communications, ICC, 2008, pp. 4048-4053.

[7] W. Zhang and U. Mitra, "A spectrum-shaping perspective on cognitive radio: Uncoded primary transmission case," in IEEE International Symposium on Information Theory, ISIT, 2008, pp. 1338-1342.

[8] I. Maric, A. Goldsmith, G. Kramer, and S. Shamai, "On the capacity of interference channels with a partially-cognitive transmitter," in IEEE International Symposium on Information Theory, ISIT, 2007, pp. 2156-2160.

[9] N. Devroye, P. Mitran, and V. Tarokh, "Achievable rates in cognitive radio channels," IEEE Trans. on Information Theory, vol. 52, no. 5, pp. 1813-1827, 2006 .

[10] W. Wu, S. Vishwanath, and A. Arapostathis, "On the capacity of gaussian weak interference channels with degraded message sets," in Information Sciences and Systems, 2006 40th Annual Conference on, 2006, pp. 1703-1708. 
[11] N. Devroye, P. Mitran, and V. Tarokh, "Limits on communications in a cognitive radio channel," IEEE Communications Magazine, vol. 44, no. 6, pp. 44-49, 2006.

[12] S. Jafar and S. Srinivasa, "Capacity limits of cognitive radio with distributed and dynamic spectral activity," IEEE Journal on Selected Areas in Communications, vol. 25, no. 3, pp. 529-537, 2007.

[13] S. Jafar, "Capacity with causal and noncausal side information: A unified view," IEEE Trans. on Information Theory, vol. 52, no. 12, pp. 5468-5474, 2006.

[14] O. Simeone, Y. Bar-Ness, and U. Spagnolini, "Stable throughput of cognitive radios with and without relaying capability," IEEE Trans. on Communications, vol. 55, no. 12, pp. 2351-2360, 2007.

[15] Z. Wu and B. Natarajan, "Interference tolerant agile cognitive radio: Maximize channel capacity of cognitive radio," in 4th IEEE Consumer Communications and Networking Conference, CCNC, 2007, pp. 1027-1031.

[16] T. Yucek and H. Arslan, "A survey of spectrum sensing algorithms for cognitive radio applications," IEEE Communications Surveys Tutorials, vol. 11, no. 1, pp. 116-130, 2009.

[17] A. Ghasemi and E. Sousa, "Collaborative spectrum sensing for opportunistic access in fading environments," in First IEEE International Symposium on New Frontiers in Dynamic Spectrum Access Networks, DySPAN 2005, Baltimore, Maryland USA, Nov. 2005, pp. $131-136$.

[18] Y. Zeng, C. Koh, and Y.-C. Liang, "Maximum eigenvalue detection: Theory and application," in Proc, of the 2008 IEEE International Conference on Communications, ICC 2008, Beijing, China, May 2008, pp. $4160-4164$.

[19] Y. Zeng and Y.-C. Liang, "Eigenvalue-based spectrum sensing algorithms for cognitive radio," IEEE Trans. on Communications, vol. 57, no. 6, pp. 1784 -1793 , June 2009.

[20] — - "Spectrum-sensing algorithms for cognitive radio based on statistical covariances," IEEE Trans. on Vehicular Technology, vol. 58, no. 4, pp. $1804-1815$, May 2009.

[21] M. Ghozzi, F. Marx, M. Dohler, and J. Palicot, "Cyclostatilonarilty-based test for detection of vacant frequency bands," in First International Conference on Cognitive Radio Oriented Wireless Networks and Communications, CROWNCOM 2006, Mykonos Island, Greece, June 2006, pp. 1 -5.

[22] Q. Zou, S. Zheng, and A. Sayed, "Cooperative sensing via sequential detection," IEEE Trans. on Signal Processing, vol. 58, no. 12, pp. 6266-6283, 2010. 
[23] D. Duan, L. Yang, and J. Principe, "Cooperative diversity of spectrum sensing for cognitive radio systems," IEEE Trans. on Signal Processing, vol. 58, no. 6, pp. 3218-3227, 2010.

[24] M. Matsui, H. Shiba, K. Akabane, and K. Uehara, "A novel cooperative sensing technique for cognitive radio," in IEEE 18th International Symposium on Personal, Indoor and Mobile Radio Communications, PIMRC2007, Athens, Greece, 2007, pp. $1-5$.

[25] J. Unnikrishnan and V. Veeravalli, "Cooperative sensing for primary detection in cognitive radio," IEEE Journal of Selected Topics in Signal Processing, vol. 2, no. 1 , pp. 18-27, 2008.

[26] Z. Quan, S. Cui, and A. Sayed, "Optimal linear cooperation for spectrum sensing in cognitive radio networks," IEEE Journal of Selected Topics in Signal Processing, vol. 2, no. 1, pp. 28-40, 2008.

[27] A. Kortun, T. Ratnarajah, M. Sellathurai, C. Zhong, and C. Papadias, "On the performance of eigenvalue-based cooperative spectrum sensing for cognitive radio," IEEE Journal of Selected Topics in Signal Processing, vol. 5, no. 1, pp. $49-55$, Feb. 2011.

[28] E. Axell, G. Leus, E. Larsson, and H. Poor, "Spectrum sensing for cognitive radio : State-of-the-art and recent advances," IEEE Signal Processing Magazine, vol. 29, no. 3, pp. 101-116, 2012.

[29] T. Cover and A. Gamal, "Capacity theorems for the relay channel," Information Theory, IEEE Transactions on, vol. 25, no. 5, pp. 572-584, 1979.

[30] J. Laneman, D. Tse, and G. Wornell, "Cooperative diversity in wireless networks: Efficient protocols and outage behavior," IEEE Trans. on Information Theory, vol. 50, no. 12, pp. 3062 - 3080, Dec. 2004.

[31] A. Nosratinia, T. Hunter, and A. Hedayat, "Cooperative communication in wireless networks," IEEE Communications Magazine, vol. 42, no. 10, pp. 74 80, Oct. 2004.

[32] T. Hunter, S. Sanayei, and A. Nosratinia, "Outage analysis of coded cooperation," IEEE Trans. on Information Theory, vol. 52, no. 2, pp. 375 - 391, Feb. 2006.

[33] J. Laneman and G. Wornell, "Distributed space-time-coded protocols for exploiting cooperative diversity in wireless networks," IEEE Trans. on Information Theory, vol. 49, no. 10, pp. 2415 - 2425, Oct. 2003.

[34] G. Scutari and S. Barbarossa, "Distributed space-time coding for regenerative relay networks," IEEE Trans. on Wireless Communications, vol. 4, no. 5, pp. 2387 - 2399, Sept. 2005. 
[35] J. Abouei, H. Bagheri, and A. Khandani, "An efficient adaptive distributed space-time coding scheme for cooperative relaying," IEEE Trans. on Wireless Communications, vol. 8, no. 10, pp. 4957 -4962, Oct. 2009.

[36] R. Nabar, H. Bolcskei, and F. Kneubuhler, "Fading relay channels: performance limits and space-time signal design," IEEE Journal on Selected Areas in Communications, vol. 22, no. 6, pp. 1099-1109, 2004.

[37] H. Ju, E. Oh, and D. Hong, "Improving efficiency of resource usage in two-hop full duplex relay systems based on resource sharing and interference cancellation," IEEE Trans. on Wireless Communications, vol. 8, no. 8, pp. 3933-3938, 2009.

[38] C. Lo, S. Vishwanath, and R. Heath, "Rate bounds for mimo relay channels using precoding," in IEEE Global Telecommunications Conference, GLOBECOM 2005, St. Louis, Missouri, USA, vol. 3, 2005, pp. 5 pp.--

[39] D. Bliss, P. Parker, and A. Margetts, "Simultaneous transmission and reception for improved wireless network performance," in IEEE/SP Workshop on Statistical Signal Processing, SSP 2007, Wiscosin, USA, 2007, pp. 478-482.

[40] S. Chen, M. Beach, and J. McGeehan, "Division-free duplex for wireless applications," Electronics Letters, vol. 34, no. 2, pp. 147-148, 1998.

[41] T. Tsiftsis, G. Karagiannidis, and S. Kotsopoulos, "Dual-hop wireless communications with combined gain relays," IEE Proceedings, Communications, vol. 152, no. 5, pp. 528-532, 2005.

[42] G. Ropokis, A. Rontogiannis, and K. Berberidis, "Ber performance analysis of cooperative daf relay networks and a new optimal daf strategy," IEEE Trans. on Wireless Communications, vol. 10, no. 4, pp. 1044-1049, 2011.

[43] A. Adinoyi and H. Yanikomeroglu, "Cooperative relaying in multi-antenna fixed relay networks," IEEE Trans. on Wireless Communications, vol. 6, no. 2, pp. 533-544, 2007.

[44] Y.-b. Kim, W. Choi, B. C. Jung, and A. Nosratinia, "A dynamic paradigm for spectrally efficient half-duplex multi-antenna relaying," IEEE Trans. on Wireless Communications, vol. 12, no. 9, pp. 4680-4691, 2013.

[45] B. Day, A. Margetts, D. Bliss, and P. Schniter, "Full-duplex mimo relaying: Achievable rates under limited dynamic range," IEEE Journal on Selected Areas in Communications, vol. 30, no. 8, pp. 1541-1553, 2012.

[46] I. Krikidis, J. Laneman, J. Thompson, and S. McLaughlin, "Protocol design and throughput analysis for multi-user cognitive cooperative systems," IEEE Trans. on Wireless Communications, vol. 8, no. 9, pp. 4740-4751, 2009. 
[47] O. Simeone, I. Stanojev, S. Savazzi, Y. Bar-Ness, U. Spagnolini, and R. Pickholtz, "Spectrum leasing to cooperating secondary ad hoc networks," IEEE Journal on Selected Areas in Communications, vol. 26, no. 1, pp. 203-213, 2008.

[48] A. Sadek, K. Liu, and A. Ephremides, "Cognitive multiple access via cooperation: Protocol design and performance analysis," IEEE Trans. on Information Theory, vol. 53, no. 10, pp. 3677-3696, 2007.

[49] O. Simeone, Y. Bar-Ness, and U. Spagnolini, "Stable throughput of cognitive radios with and without relaying capability," IEEE Trans. on Communications, vol. 55, no. 12, pp. 2351-2360, 2007.

[50] V. Havary-Nassab, S. ShahbazPanahi, A. Grami, and Z.-Q. Luo, "Distributed beamforming for relay networks based on second-order statistics of the channel state information," IEEE Trans. on Signal Processing, vol. 56, no. 9, pp. 4306 -4316 , Sept. 2008.

[51] S. Kim, J.-H. Park, and D.-J. Park, "Beamforming of amplify-and-forward relays under individual power constraints," IEEE Journal on Selected Areas in Communications, vol. 30, no. 8, pp. 1347 -1357, Sept. 2012.

[52] J. Li, A. Petropulu, and H. Poor, "Cooperative transmission for relay networks based on second-order statistics of channel state information," IEEE Trans. on Signal Processing, pp. 1280 -1291, March 2011.

[53] Y. Jing and H. Jafarkhani, "Network beamforming using relays with perfect channel information," IEEE Transactions on Information Theory, vol. 55, no. 6, pp. 2499-2517, June.

[54] G. Zheng, K.-K. Wong, A. Paulraj, and B. Ottersten, "Collaborative-relay beamforming with perfect csi: Optimum and distributed implementation," IEEE Signal Processing Letters, vol. 16, no. 4, pp. 257-260, April.

[55] J. Liu, W. Chen, Z. Cao, and Y. Zhang, "A distributed beamforming approach for enhanced opportunistic spectrum access in cognitive radios," in IEEE Global Telecommunications Conference, GLOBECOM 2009, Atlanta, USA, 2009, pp. $1-6$.

[56] H. Zhang, N. Mehta, A. Molisch, J. Zhang, and H. Dai, "Asynchronous interference mitigation in cooperative base station systems," IEEE Trans. on Wireless Communications, vol. 7, no. 1, pp. 155-165, 2008.

[57] M. Hassan and M. Hossain, "Cooperative beamforming for cognitive radio systems with asynchronous interference to primary user," IEEE Trans. on Wireless Communications, vol. to appear, no. 99, pp. 1-12, 2013.

[58] D. Bertsekas, "A new class of incremental gradient methods for least squares problems," newblock SIAM J. Optim., vol. 7, no. 99, pp. 913-926, Nov. 1997. 
[59] M. Rabbat and R. Nowak, "Quantized incremental algorithms for distributed optimization," IEEE Journal on Selected Areas in Communications, vol. 23, no. 4, pp. 798-808, 2005.

[60] C. Lopes and A. Sayed, "Distributed adaptive incremental strategies: Formulation and performance analysis," in IEEE International Conference on Acoustics, Speech and Signal Processing, ICASSP 2006, Toulouse, France, vol. 3, 2006, pp. III-III.

[61] J. Predd, S. Kulkarni, and H. Poor, "Distributed learning in wireless sensor networks," IEEE Signal Processing Magazine, vol. 23, no. 4, pp. 56-69, 2006.

[62] K. Srivastava and A. Nedic, "Distributed asynchronous constrained stochastic optimization," IEEE Journal of Selected Topics in Signal Processing, vol. 5, no. 4, pp. 772-790, 2011.

[63] J. Chen and A. Sayed, "Diffusion adaptation strategies for distributed optimization and learning over networks," IEEE Trans. on Signal Processing, vol. 60, no. 8, pp. 4289-4305, 2012.

[64] S. Chouvardas, K. Slavakis, and S. Theodoridis, "Adaptive robust distributed learning in diffusion sensor networks," IEEE Trans. on Signal Processing, vol. 59, no. 10, pp. 4692-4707, 2011.

[65] J. Tsitsiklis, D. Bertsekas, and M. Athans, "Distributed asynchronous deterministic and stochastic gradient optimization algorithms," IEEE Trans. on Automatic Control, vol. 31, no. 9, pp. 803-812, 1986.

[66] S. Kar and J. Moura, "Convergence rate analysis of distributed gossip (linear parameter) estimation: Fundamental limits and tradeoffs," IEEE Journal of Selected Topics in Signal Processing, vol. 5, no. 4, pp. 674-690, 2011.

[67] I. Schizas, G. Mateos, and G. Giannakis, "Distributed lms for consensus-based in-network adaptive processing," IEEE Trans. on Signal Processing, vol. 57, no. 6, pp. 2365-2382, 2009.

[68] S. Barbarossa and G. Scutari, "Bio-inspired sensor network design," IEEE Signal Processing Magazine, vol. 24, no. 3, pp. 26-35, 2007.

[69] S.-Y. Tu and A. Sayed, "Diffusion strategies outperform consensus strategies for distributed estimation over adaptive networks," IEEE Trans. on Signal Processing, vol. 60, no. 12, pp. 6217-6234, 2012.

[70] C. Tsinos and K. Berberidis, "Blind opportunistic interference alignment in cognitive radio systems," in IEEE International Conference on Communications, ICC 2013, Budapest, Hungary, June 2013. 
[71] — _ "Blind opportunistic interference alignment in MIMO cognitive radio systems," IEEE Journal on Emerging and Selected Topics in Circuits and Systems, vol. 3, no. 4, to appear, December 2013.

[72] — - "Adaptive eigenvalue-based spectrum sensing for multi-antenna cognitive radio systems," in IEEE International Conference on on Acoustics, Speech and Signal Processing, ICASSP 2013, Vancouver, Canada, June 2013.

[73] — - "submitted todecentralized adaptive eigenvalue-based spectrum sensing for multi-antenna cognitive radio systems," submitted to the IEEE Trans. on Wireless Communications, October 2013.

[74] — _ "A new cooperative technique for wireless communications with improved diversity-multiplexing tradeoff," in European Signal Processing Conference, EUSIPCO 2009, Glasgow, Scotland, August 2009.

[75] — - "A cooperative uplink transmission technique for the single- and multiuser case," in IEEE International Conference on Communications, ICC-2010, Cape Town, South Africa, June 2010, pp. 1-5.

[76] — - "A cooperative uplink transmission technique with improved diversitymultiplexing tradeoff," submitted to the IEEE Trans. on Communications, October 2013.

[77] C. Tsinos, E. Vlachos, and K. Berberidis, "Distributed blind adaptive computation of beamforming weights for relay networks,," in IEEE International Symposium on Personal, Indoor and Mobile Radio Communications, PIMRC 2013, London, UK, Semptember 2013.

[78] V. Cadambe and S. Jafar, "Interference alignment and degrees of freedom of the k -user interference channel," IEEE Trans. on Information Theory, vol. 54, no. 8, pp. $3425-3441$, Aug. 2008.

[79] S. Peters and R. Heath, "Cooperative algorithms for MIMO interference channels," IEEE Trans. on Vehicular Technology, vol. 60, no. 1, pp. 206-218, Jan. 2011.

[80] K. Gomadam, V. Cadambe, and S. Jafar, "A distributed numerical approach to interference alignment and applications to wireless interference networks," IEEE Trans. on Information Theory, vol. 57, no. 6, pp. 3309 -3322, June 2011.

[81] S. Perlaza, N. Fawaz, S. Lasaulce, and M. Debbah, "From spectrum pooling to space pooling: Opportunistic interference alignment in MIMO cognitive networks," IEEE Trans. on Signal Processing, vol. 58, no. 7, pp. 3728 -3741, July 2010.

[82] D. Tse and P. Viswanath, Fundamentals of Wireless Communications. Cambridge University Press, 2004. 
[83] O. El Ayach, A. Lozano, and R. Heath, "On the overhead of interference alignment: Training, feedback, and cooperation," IEEE Trans. on Wireless Communications, vol. 11, no. 11, pp. 4192-4203, 2012.

[84] B. Nosrat-Makouei, J. Andrews, and R. Heath, "MIMO interference alignment over correlated channels with imperfect CSI," IEEE Trans. on Signal Processing, vol. 59, no. 6, pp. 2783 -2794, June 2011.

[85] S. Jayaweera and H. Poor, "Capacity of multiple-antenna systems with both receiver and transmitter channel state information," IEEE Trans. on Information Theory, vol. 49, no. 10, pp. 2697 - 2709, Oct. 2003.

[86] Z. Shen, J. Heath, J. Andrews, and B. Evans, "Comparison of space-time waterfilling and spatial water-filling for MIMO fading channels," in Proc. of the 2004 IEEE Global Telecommunications Conference, GLOBECOM-2004, Dec. 2004, Dallas, USA., vol. 1.

[87] L. C. Andrews, Special Functions for Engineers and Applied Mathematicians. MacMillan, 1985.

[88] T. Taniguchi, S. Sha, and Y. Karasawa, "Statistical distribution of eigenvalues of correlation matrices in i.i.d MIMO channels under rayleigh fading," in Proc. of the 16th IEEE International Symposium on Personal, Indoor and Mobile Radio Communications, 2005. PIMRC 2005, vol. 1, Sept. 2005, Berlin, Germany, pp. $537-541$.

[89] M. Wax and T. Kailath, "Detection of signals by information theoretic criteria," IEEE Trans. on Acoustics, Speech and Signal Processing, vol. 33, no. 2, pp. 387 - 392, Apr. 1985.

[90] A. Shah and D. Tufts, "Determination of the dimension of a signal subspace from short data records," IEEE Trans. on Signal Processing, vol. 42, no. 9, pp. $2531-2535$, Sept. 1994.

[91] S. Kritchman and B. Nadler, "Determining the number of components in a factor model from limited noisy data," Chemometrics and Intelligent Laboratory Systems, vol. 94, no. 1, pp. 19-32, 2008.

[92] C. Cormio and K. R. Chowdhury, "A survey on mac protocols for cognitive radio networks," Ad Hoc Netw., vol. 7, no. 7, pp. 1315-1329, Sep. 2009.

[93] X. G. Doukopoulos and G. V. Moustakides, "Fast and stable subspace tracking," IEEE Trans. on Signal Processing, vol. 56, no. 4, pp. 1452-1465, Apr. 2008.

[94] T. Yoo and A. Goldsmith, "Capacity and power allocation for fading mimo channels with channel estimation error," IEEE Trans. on Information Theory, vol. 52, no. 5, pp. $2203-2214$, May 2006. 
[95] B. Friedlander and A. Weiss, "On the second-order statistics of the eigenvectors of sample covariance matrices," IEEE Trans. on Signal Processing, vol. 46, no. 11, pp. 3136 -3139, Nov. 1998.

[96] M. Kaveh and A. Barabell, "The statistical performance of the music and the minimum-norm algorithms in resolving plane waves in noise," IEEE Trans. on Acoustics, Speech and Signal Processing, vol. 34, no. 2, pp. 331 -341, Apr. 1986.

[97] F. Penna, R. Garello, D. Figlioli, and M. Spirito, "Exact non-asymptotic threshold for eigenvalue-based spectrum sensing," in Proc. of the Fourth International Conference on Cognitive Radio Oriented Wireless Networks and Communications, CROWNCOM 2009 ,Hannover, Germany, June 2009, pp. 1-5.

[98] L. Li, A. Scaglione, and J. Manton, "Distributed principal subspace estimation in wireless sensor networks," IEEE Journal of Selected Topics in Signal Processing, vol. 5, no. 4, pp. 725 -738, Aug. 2011.

[99] C. Reyes, T. Hilaire, and C. Mecklenbrauker, "Distributed projection approximation subspace tracking based on consensus propagation," in 3rd IEEE International Workshop on Computational Advances in Multi-Sensor Adaptive Processing CAMSAP 2009, Aruba, Dutch Antilles, Dec. 2009, pp. 340 -343.

[100] A. Taherpour, M. Nasiri-Kenari, and S. Gazor, "Multiple antenna spectrum sensing in cognitive radios," IEEE Trans. on Wireless Communications, vol. 9, no. 2, pp. $814-823$, Feb. 2010.

[101] P. Wang, J. Fang, N. Han, and H. Li, "Multiantenna-assisted spectrum sensing for cognitive radio," IEEE Trans. on Vehicular Technology, vol. 59, no. 4, pp. $1791-1800$, May 2010.

[102] B. Nadler, F. Penna, and R. Garello, "Performance of eigenvalue-based signal detectors with known and unknown noise level," in Proc. of the 2011 IEEE International Conference on Communications ICC 2011, Kyoto, Japan, June 2011, pp. $1-5$.

[103] B. L. Welch, "The significance of the difference between two means when the population variances are unequal," Biometrika, vol. 29, no. 3/4, pp. pp. 350-362, 1938. [Online]. Available: http://www.jstor.org/stable/2332010

[104] A. Sayed and C. Lopes, "Distributed processing over adaptive networks," in 9th International Symposium on Signal Processing and Its Applications, ISSPA 2007, Sharjah, U.A.E., Feb. 2007, pp. 1 -3.

[105] G. H. Golub and C. F. Van Loan, Matrix computations (3rd ed.). Baltimore, MD, USA: Johns Hopkins University Press, 1996.

[106] N. L. Johnson, S. Kotz, and N. Balakrishnan, Continuous Univariate Distributions, Vol. 1 (Wiley Series in Probability and Statistics), 2nd ed. WileyInterscience. [Online]. Available: http://www.worldcat.org/isbn/0471584959 
[107] Y. Fan, C. Wang, J. Thompson, and H. Poor, "Recovering multiplexing loss through successive relaying using repetition coding," IEEE Trans. on Wireless Communications, vol. 6, no. 12, pp. 4484 -4493, Dec. 2007.

[108] S. Yang and J.-C. Belfiore, "Towards the optimal amplify-and-forward cooperative diversity scheme," IEEE Trans. on Information Theory, vol. 53, no. 9, pp. $3114-3126$, Sept. 2007.

[109] K. Azarian, H. El Gamal, and P. Schniter, "On the achievable diversitymultiplexing tradeoff in half-duplex cooperative channels," IEEE Trans. on Information Theory, vol. 51, no. 12, pp. 4152 -4172, Dec. 2005.

[110] E. Larsson and B. Vojcic, "Cooperative transmit diversity based on superposition modulation," IEEE Communications Letters, vol. 9, no. 9, pp. 778 - 780, Sept. 2005.

[111] Z. Ding, T. Ratnarajah, and C. Cowan, "On the diversity-multiplexing tradeoff for wireless cooperative multiple access systems," IEEE Trans. on Signal Processing, vol. 55, no. 9, pp. 4627 -4638, Sept. 2007.

[112] L. Zheng and D. Tse, "Diversity and multiplexing: a fundamental tradeoff in multiple-antenna channels," IEEE Trans. on Information Theory, vol. 49, no. 5, pp. 1073 - 1096, May 2003.

[113] A. Bletsas, A. Khisti, D. Reed, and A. Lippman, "A simple cooperative diversity method based on network path selection," IEEE Journal on Selected Areas in Communications, vol. 24, no. 3, pp. 659-672, 2006.

[114] Y. Zou, Y.-D. Yao, and B. Zheng, "Opportunistic distributed space-time coding for decode-and-forward cooperation systems," IEEE Trans. on Signal Processing, vol. 60, no. 4, pp. 1766 -1781, April 2012.

[115] M. K. Simon and M.-S. Alouini, Digital Communication over Fading Channels (Wiley Series in Telecommunications and Signal Processing). Wiley-IEEE Press, Dec. 2004.

[116] I. Ansari, F. Yilmaz, M.-S. Alouini, and O. Kucur, "On the sum of gamma random variates with application to the performance of maximal ratio combining over nakagami-m fading channels," in IEEE 13th International Workshop on Signal Processing Advances in Wireless Communications (SPAWC), 2012, pp. 394-398.

[117] V. Aalo, T. Piboongungon, and G. Efthymoglou, "Another look at the performance of mrc schemes in nakagami-m fading channels with arbitrary parameters," IEEE Trans. on Communications, vol. 53, no. 12, pp. 2002-2005, 2005.

[118] H. Exton, Multiple Hypergeometric Functions and Applications. Wiley, 1976. 
[119] A. M. Mathai, R. K. Saxena, and H. Haubold, The H-function With Applications in Statistics and Other Disciplines. Springer, 2010.

[120] S. Shahbazpanahi, A. B. Gershman, Z.-Q. Luo, and K. M. Wong, "Robust adaptive beamforming for general-rank signal models," IEEE Transactions on Signal Processing, vol. 51, no. 9, pp. 2257-2269, 2003. 